\title{
Visible Light-Induced Reductive Alkynylation of Aldehydes by Umpolung Approach
}

\author{
Ibuki Tanaka, ${ }^{\ddagger}$ Masaya Sawamura, ${ }^{*}, \dagger, \downarrow$ and Yohei Shimizu ${ }^{*}, \dagger, \dagger$
}

$[\dagger]$ Institute for Chemical Reaction Design and Discovery (WPI-ICReDD), Hokkaido University, Kita 21 Nishi 10, Kita-ku, Sapporo, Hokkaido 001-0021, Japan

[†] Department of Chemistry, Faculty of Science, Hokkaido University, Kita 10 Nishi 8, Kita-ku, Sapporo, Hokkaido 060-0810, Japan

$$
\text { E-mail: } \begin{aligned}
& \text { sawamura@sci.hokudai.ac.jp } \\
& \underline{\text { shimizu-y@sci.hokudai.ac.jp }}
\end{aligned}
$$




\section{Supporting Information}

Table of Contents

$\begin{array}{ll}\text { Instrumentation and chemicals } & \text { S1 }\end{array}$

$\begin{array}{ll}\text { Typical procedures for reductive alkynylation of aldehydes } & \text { S2 }\end{array}$

$\begin{array}{ll}\text { Optimization } & \text { S2-S6 }\end{array}$

$\begin{array}{ll}\text { Cyclic voltammetry } & \text { S7 }\end{array}$

$\begin{array}{ll}\text { Characterization of alkynylsulfones } & \text { S8-S9 }\end{array}$

$\begin{array}{ll}\text { Characterization of products } & \text { S9-S18 }\end{array}$

$\begin{array}{lr}\text { References } & \text { S19 }\end{array}$

$\begin{array}{ll}\text { NMR Spectra } & \text { S20-S65 }\end{array}$

\section{Instrumentation and chemicals}

NMR spectra were recorded on a JEOL ECX-400, operating at $400 \mathrm{MHz}$ for ${ }^{1} \mathrm{H}$ NMR and 100.5 $\mathrm{MHz}$ for ${ }^{13} \mathrm{C}$ NMR. Chemical shift values for ${ }^{1} \mathrm{H}$ and ${ }^{13} \mathrm{C}$ are referenced to $\mathrm{Me} 4 \mathrm{Si}$ or a residual solvent peak. Chemical shifts are reported in $\delta$ ppm. Mass spectra were obtained with JEOL JMST100GCV or Thermo Scientific Exactive at Global Facility Center, Creative Research Institution, Hokkaido University. TLC analyses were performed on commercial glass plates bearing 0.25-mm layer of Merck Silica gel 60F 254 . Silica gel (Kanto Chemical Co., Silica gel 60 N, spherical, neutral) was used for column chromatography. Biotage Isolera One with packed column was also used for normal phase column chromatography.

All reactions were carried out under nitrogen or argon atmosphere. Materials were obtained from commercial suppliers or prepared according to standard procedures unless otherwise noted. All the aldehydes were recrystallized or distilled before use. All solvents for catalytic reactions were degassed and stored over molecular sieves $4 \AA$ in a glove box. Hantzsch ester was prepared according to the reported procedure. ${ }^{1}$

Photochemical Reactor Aldrich ${ }^{\circledR}$ Micro Photochemical Reactor, blue LED lights (ALDKIT0011EA). LED light is IP68 double density $12 \mathrm{vdc}$ water proof blue light with spectral range of 435-445 $\mathrm{nm}$ with wall plug power supply $500 \mathrm{~mA}$ with 5-6 watts. The irradiation vessel material is borosilicate glass. The distance of irradiation vessel from light source is $2 \mathrm{~cm}$. There is no use of any kind of filters. 


\section{Typical procedures for reductive alkynylation of aldehydes}
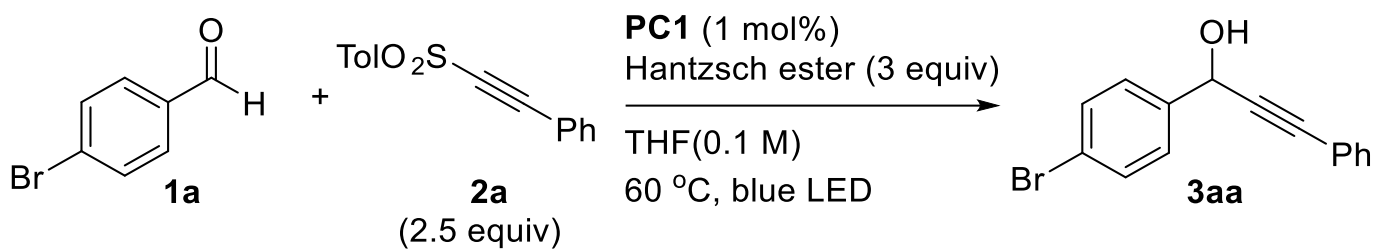

(2.5 equiv)

In a glove box filled with nitrogen gas, a rubber capped screw vial containing magnetic stirring bar was charged with 1a (18.5 mg, $0.1 \mathrm{mmol}), 2 \mathrm{a}(64.1 \mathrm{mg}, 0.25 \mathrm{mmol}), \mathbf{P C 1}(1.1 \mathrm{mg}, 0.001 \mathrm{mmol})$, Hantzsch ester $(76.0 \mathrm{mg}, 0.3 \mathrm{mmol})$, and THF $(1 \mathrm{~mL})$. The mixture was taken out from the glove box and stirred for $3 \mathrm{~h}$ at $60{ }^{\circ} \mathrm{C}$ using a hot stirrer equipped with an aluminum block under blue LED (Aldrich ${ }^{\circledR}$ Micro Photochemical Reactor, blue LED lights) irradiation. The reaction mixture was evaporated and passed through short pad of silica gel with EtOAc. Eluted mixture was concentrated under reduced pressure to afford the crude product. The crude product was purified by preparative TLC (hexane/EtOAc 2:1) to give 3aa (24.4 mg, 84\% yield) as colorless solid.

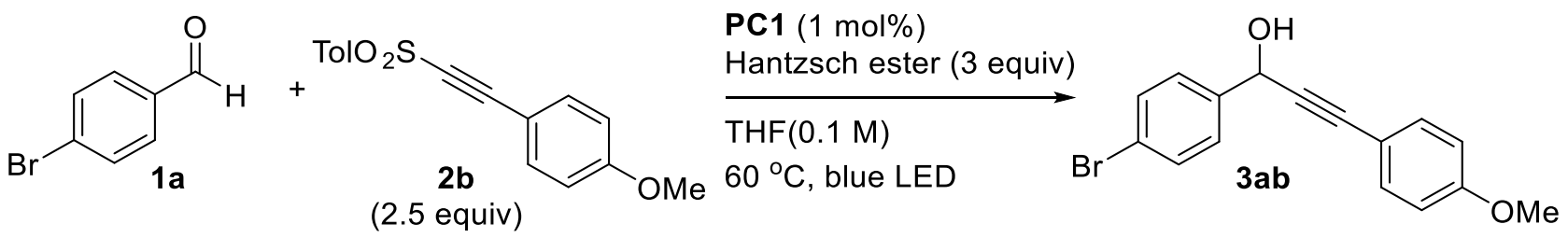

In a glove box filled with nitrogen gas, a screw vial was charged with 1a (185 mg, $1 \mathrm{mmol}), \mathbf{2 b}$ (716 mg, $2.5 \mathrm{mmol})$, PC1 (11 mg, $0.01 \mathrm{mmol})$, Hantzsch ester (760 mg, $3.0 \mathrm{mmol})$, and THF (10 $\mathrm{mL}$ ). The mixture was divided to 10 rubber capped screw vials (approximately $1 \mathrm{~mL}$ each) and taken out from the glove box and stirred for $3 \mathrm{~h}$ at $60{ }^{\circ} \mathrm{C}$ using a hot stirrer equipped with an aluminum block under blue LED (Aldrich ${ }^{\circledR}$ Micro Photochemical Reactor, blue LED lights) irradiation. The reaction mixture was combined, evaporated and passed through short pad of silica gel with EtOAc. Eluted mixture was concentrated under reduced pressure to afford the crude product. The crude product was purified by silica gel column chromatography (hexane/EtOAc 2:1) to give $\mathbf{3 a b}$ ( $275 \mathrm{mg}, 87 \%$ yield) as colorless solid.

\section{Optimization}

\section{Photocatalysts}

Investigation of photocatalyst was conducted using Hantzsch ester as terminal reductant as well as proton donor (Table S1). The result indicates the importance of reduction potential of the photocatalyst. If the potential is too small, the product 3aa was obtained in at most 5\% yield (entries 1-3). This result suggests that the reduction potentials of these photocatalysts are not sufficient to 
induce single-electron reduction of 1a. On the other hand, when the potential is higher than PC1, the product yield was diminished (entries 5 and 6). Observation of an alkenylsulfone derived from 2a by GCMS analysis in these reactions (Figure S1) indicated that the competitive reduction of 2a over desired reduction of 1 a might cause the decrease in the product yield.

Table S1. Photocatalysts<smiles>O=Cc1ccc(Br)cc1</smiles>

$(0.1 \mathrm{mmol})$<smiles>O[PbH2]OC#CPc1ccccc1</smiles>

$2 a$

(3 equiv) photocatalyst (1 mol\%)

Hantzsch ester (3 equiv)

$\operatorname{THF}(0.1 \mathrm{M})$

$\mathrm{rt}, 3 \mathrm{~h}$, blue LED<smiles>OC(C#Cc1ccccc1)c1ccc(Br)cc1</smiles>

3aa

\begin{tabular}{|c|c|c|c|}
\hline entry & photocatalyst & reduction potential & yield $(\%)$ \\
\hline 1 & {$\left[\mathrm{Ru}(\mathrm{bpz})_{3}\right]\left[\mathrm{PF}_{6}\right]_{2}$} & $\mathrm{Ru}(\mathrm{III}) / \mathrm{Ru}(\mathrm{II})=-0.80 \mathrm{~V}^{3,4}$ & 0 \\
\hline 2 & Eosin $Y$ & $\mathrm{E}_{1 / 2}\left(\mathrm{P} / \mathrm{P}^{-}\right)=-1.08 \mathrm{~V}^{5}$ & 0 \\
\hline 3 & 4CzIPN (PC3) & $\mathrm{E}_{1 / 2}\left(\mathrm{P} / \mathrm{P}^{-}\right)=-1.21 \mathrm{~V}^{6}$ & 5 \\
\hline 4 & {$\left[\operatorname{Ir}\left(\mathrm{dF}-\mathrm{CF}_{3}-\mathrm{ppy}\right)_{2}(\mathrm{dtbpy})\right] \mathrm{PF}_{6}(\mathbf{P C 1})$} & $\operatorname{Ir}(\mathrm{III}) / \operatorname{Ir}(\mathrm{II})=-1.37 \mathrm{~V}^{3,7}$ & 22 \\
\hline 5 & {$\left[\operatorname{Ir}(\mathrm{ppy})_{2}(\mathrm{dtbpy})\right] \mathrm{PF}_{6}(\mathbf{P C 2})$} & $\operatorname{Ir}(\mathrm{III}) / \operatorname{Ir}(\mathrm{II})=-1.51 \mathrm{~V}^{3,7}$ & 16 \\
\hline 6 & {$\left[\operatorname{Ir}(\mathrm{ppy})_{3}\right]$} & $\operatorname{Ir}(\mathrm{III}) / \operatorname{Ir}(\mathrm{II})=-2.19 \mathrm{~V}^{3}$ & 14 \\
\hline
\end{tabular}

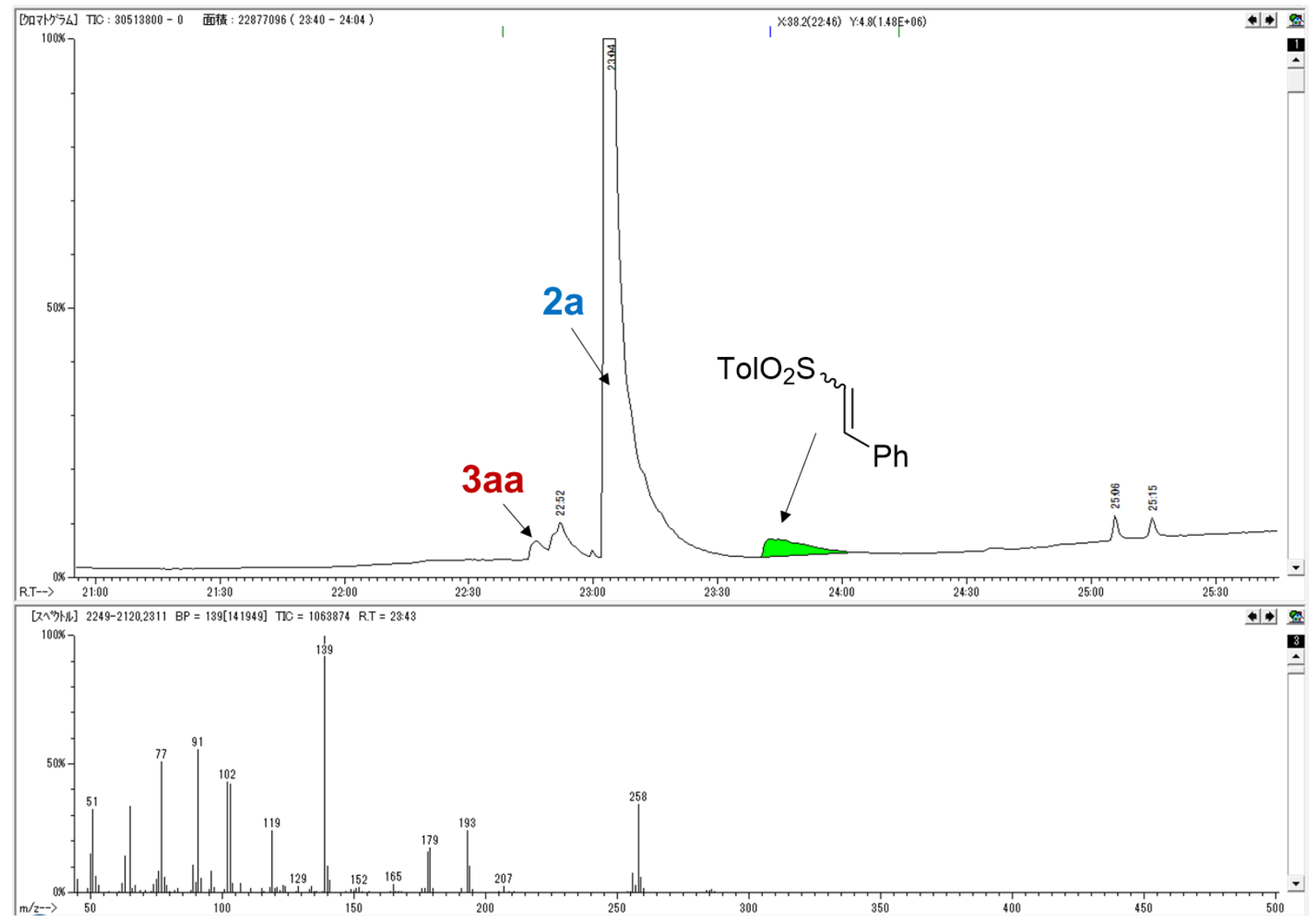

Figure S1. GCMS (EI) analysis of crude mixture of entry 5 in Table S1. 


\section{Terminal reductants}

Investigation of terminal reductants was conducted using PC1 as the photocatalyst (Table S2). Although there are several precedents which use secondary or tertiary amine as both terminal reductant and proton source in ketyl radical generation by photocatalyst, neither of them was applicable in this reaction.

\section{Table S2. Terminal reductants}<smiles>O=Cc1ccc(Br)cc1</smiles>

(0.1 mmol)
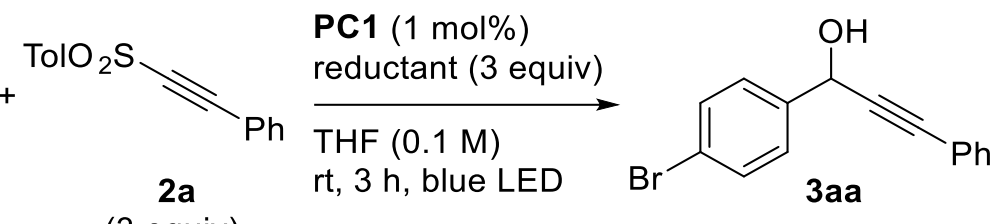

(3 equiv)

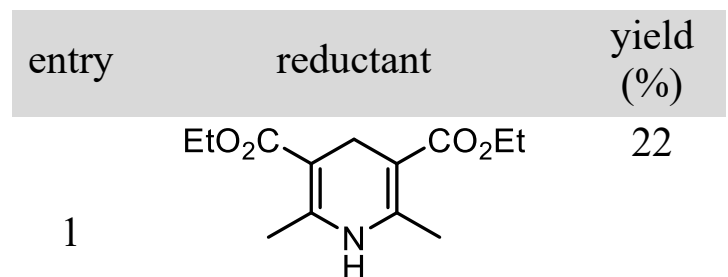

2

Hantzsch ester

2

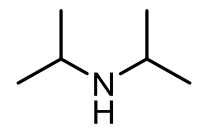<smiles>CCN(C(C)C)C(C)C</smiles><smiles>CN1c2ccccc2SC1c1ccccc1</smiles> 


\section{Temperature}

When the temperature was increased to $40{ }^{\circ} \mathrm{C}$ from room temperature, the product yield was significantly improved to $79 \%$ (Table S3, entry 2) from $22 \%$ (entry 1 ). The highest $84 \%$ yield was obtained at $60{ }^{\circ} \mathrm{C}$ (entry 3 ). Because $\mathbf{2 a}$ and Hantzsch ester were fully soluble only at the elevated temperature, the optical transparency was not good at room temperature, leading to diminished efficiency of photoexcitation of PC1.

Table S3. Temperature

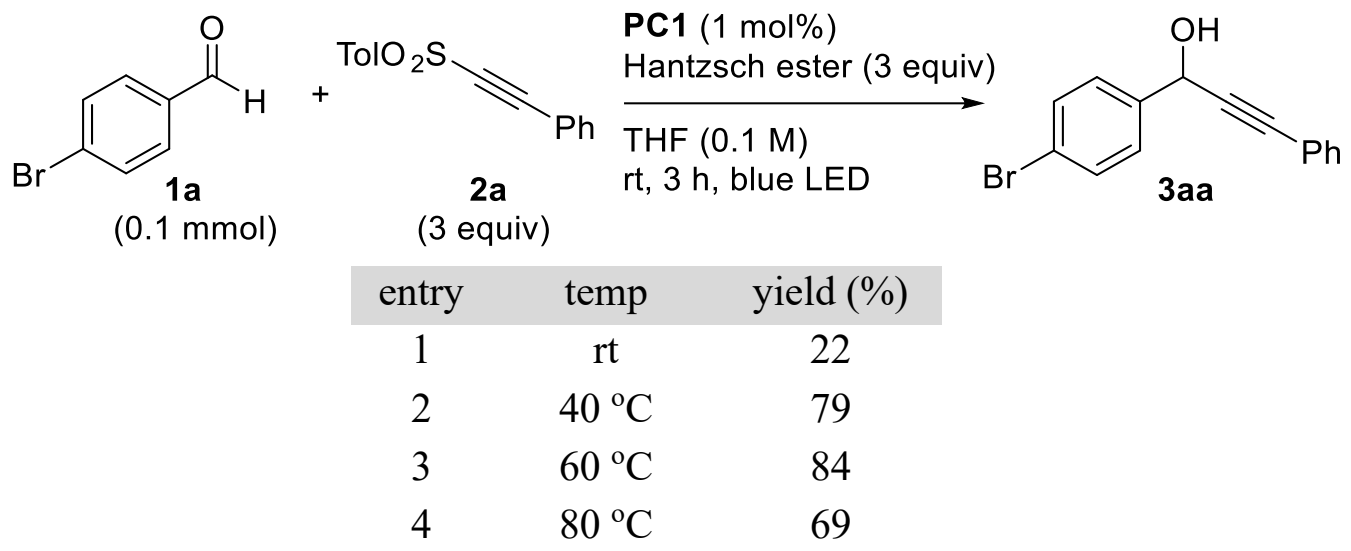

\section{Base or acid additives}

Influence of several additives was examined (Table S4). Addition of $\mathrm{K}_{2} \mathrm{CO}_{3}$ or $\mathrm{N}$-Me-pyrrolidine completely inhibited the reaction. In contrast, addition of an acid, TFA, did not show meaningful effect. These results are consistent with the involvement of PCET in this reaction. ${ }^{8}$

Table S4. Additives

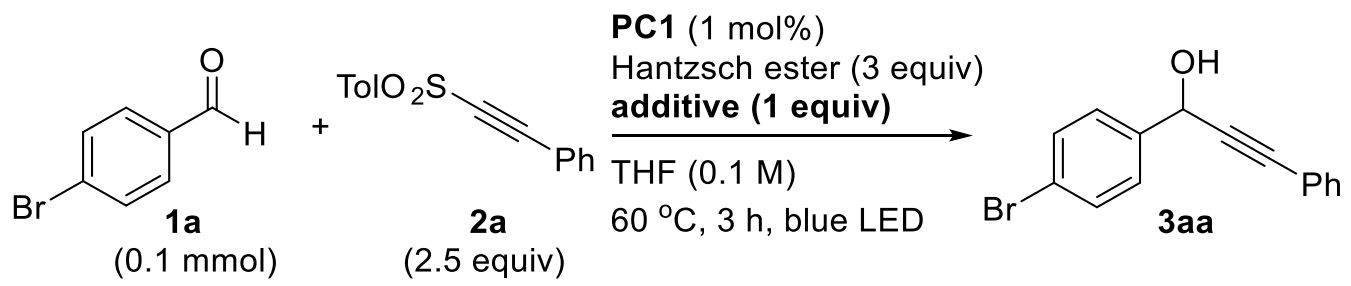

$\begin{array}{ccc}\text { entry } & \text { additive } & \text { yield }(\%) \\ 1 & - & 92 \\ 2 & \mathrm{~K}_{2} \mathrm{CO}_{3} & 0 \\ 3 & N \text {-Me-pyrrolidine } & 0 \\ 4 & \text { TFA } & 86\end{array}$




\section{Amount of 2a and Hantzsch ester}

When less than 2.5 equivalents of 2a was added (Table S5, entries 2 and 3), the yields of 3aa decreased. Likewise, excess amount of 2 a (3 equiv in entry 4) also decreased the yield. Although the amount of Hantzsch ester was not so critical to the product yields (entries 5 and 6), the best yield was obtained with 3 equivalents of Hantzsch ester (entry 1).

\section{Table S5. Amount of reagents}

\begin{tabular}{|c|c|c|c|c|}
\hline O & & $\begin{array}{l}\text { PC1 }(1 \mathrm{~mol} \%) \\
\text { Hantzsch ester (y equ }\end{array}$ & & \\
\hline $1 \mathrm{a}$ & $\begin{array}{c}2 a \\
\text { (x equiv) }\end{array}$ & $\begin{array}{l}\text { THF }(0.1 \mathrm{M}) \\
60^{\circ} \mathrm{C}, 3 \mathrm{~h} \text {, blue LED }\end{array}$ & & $3 a a$ \\
\hline entry & $\begin{array}{c}\mathbf{2 a} \\
\text { (equiv) }\end{array}$ & $\begin{array}{l}\text { Hantzsch ester } \\
\text { (equiv) }\end{array}$ & $\begin{array}{l}\text { yield } \\
(\%)\end{array}$ & \\
\hline 1 & 2.5 & 3 & 92 & \\
\hline 2 & 1.5 & 3 & 79 & \\
\hline 3 & 2 & 3 & 85 & \\
\hline 4 & 3 & 3 & 83 & \\
\hline 5 & 2.5 & 2 & 87 & \\
\hline 6 & 2.5 & 4 & 90 & \\
\hline
\end{tabular}




\section{Cyclic voltammetry}

Information of electronic properties of 2-methylbenzaldehyde $\mathbf{1 h}$ and alkynylsulfone $\mathbf{2 a}$ was obtained by cyclic voltammetry in $\mathrm{MeCN}$ containing $\mathrm{Bu}_{4} \mathrm{PF}_{6}(0.1 \mathrm{M})$ as a supporting electrolyte (scan rate $0.1 \mathrm{Vs}^{-1}$, glassy carbon electrode) (Figure S1). 1h (Figure S2a) and 2a (Figure S2b) showed voltammograms with reduction peaks at $-1.91 \mathrm{~V}$ for $\mathbf{1 h}$ and $-1.68 \mathrm{~V}$ for $\mathbf{2 a}$, respectively. This result suggests that alkynylsulfone $\mathbf{2 a}$ is much more susceptible to single-electron reduction than aldehyde $\mathbf{1 h}$. However, generation of propargyl alcohol 3ha in Table 2 indicates occurrence of reduction of $\mathbf{1 h}$ in the presence of $\mathbf{2 a}$ to form the ketyl radical for alkynylation. This discrepancy can be attributed to PCET which facilitates single-electron reduction of $\mathbf{1 h}$ over $\mathbf{2 a}$ in the reaction mixture, where the protic hydrogen of a pyridinium cation derived from Hantzsch ester activates $\mathbf{1 h}$. Indeed, the voltammogram of $\mathbf{1} \mathbf{h}$ in the presence of protic acid TFA (1equiv) exhibited significant change (Figure S2c), highlighting the appearance of new reduction peak at $-1.34 \mathrm{~V}$. The voltammogram of $\mathbf{2 a}$ in the presence of TFA (1 equiv) also showed new reduction peak at $-1.38 \mathrm{~V}$ (Figure S2d), comparable but less reducible value than that of $\mathbf{1 h}$ with TFA. The results suggest that single-electron reduction of $\mathbf{1 h}$ and $\mathbf{2 a}$ can be competitive in the presence of proton source, while the reduction of $\mathbf{1 h}$ is slightly preferrable to afford the ketyl radical.

(a)

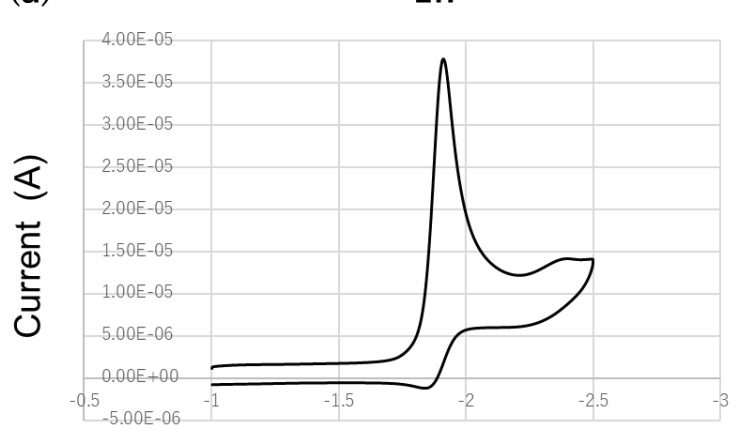

$E(V)$ vs. SCE

(c)

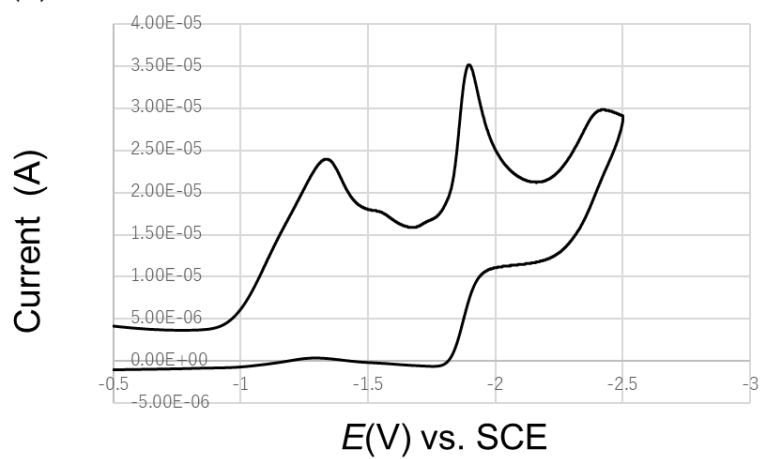

(b)

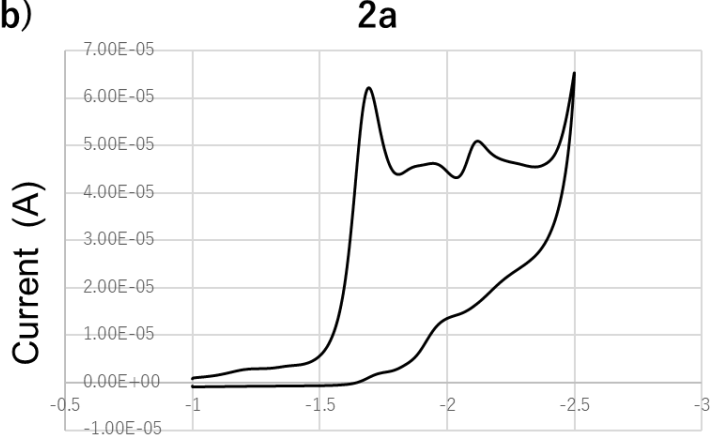

$E(V)$ vs. SCE

(d)

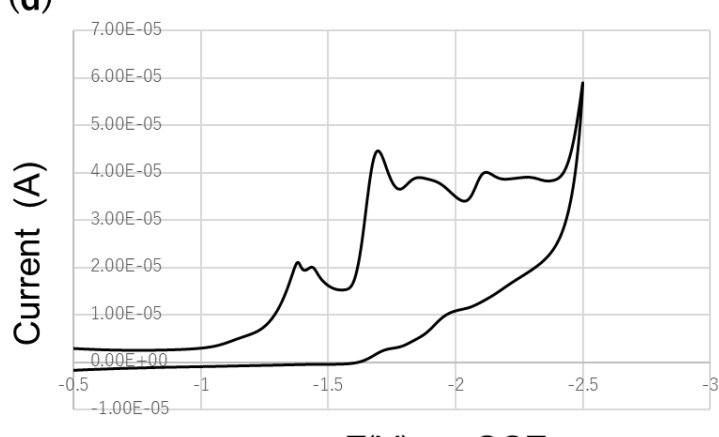

$E($ V) vs. SCE

Figure S2. Cyclic voltammogram. Substrates in $\mathrm{MeCN}$ containing $\mathrm{Bu}_{4} \mathrm{PF}_{6}(0.1 \mathrm{M})$ as a supporting electrolyte (scan rate $0.1 \mathrm{Vs}^{-1}$, glassy carbon electrode). (a) $\mathbf{1 h}(1 \mathrm{mM})$ (b) $\mathbf{2 a}(1$ $\mathrm{mM})$ (c) $\mathbf{1 h}(1 \mathrm{mM})$, TFA (1 mM) (d) $\mathbf{2 a}(1 \mathrm{mM})$, TFA (1 mM). 


\section{Characterization of alkynylsulfones}

Alkynylsulfones were prepared according to the reported procedure. ${ }^{9}$

2-Methoxy-6-(tosylethynyl)naphthalene (2d)

$\mathrm{TolO}_{2} \mathrm{~S}$<smiles>CC#Cc1ccc2cc(OC)ccc2c1</smiles>

2d

$259.1 \mathrm{mg}$, 39\% yield, colorless solid. Mp $136.8-137.8{ }^{\circ} \mathrm{C}$.

Rf: 0.25 (EtOAc/Hexane 2:8).

${ }^{1} \mathbf{H}$ NMR $\left(400 \mathrm{MHz}, \mathrm{CDCl}_{3}\right) \delta 8.02-7.96(\mathrm{~m}, 3 \mathrm{H}), 7.70(\mathrm{~d}, J=4.4 \mathrm{~Hz}, 1 \mathrm{H}), 7.68(\mathrm{~d}, J=4.4 \mathrm{~Hz}, 1 \mathrm{H})$, $7.45(\mathrm{~d}, J=8.8 \mathrm{~Hz}, 1 \mathrm{H}), 7.39(\mathrm{~d}, J=8.0 \mathrm{~Hz}, 2 \mathrm{H}), 7.18(\mathrm{~d}, J=9.2 \mathrm{~Hz}, 1 \mathrm{H}), 7.10(\mathrm{~s}, 1 \mathrm{H}), 3.93$ (s, $3 \mathrm{H}), 2.47$ (s, 3H).

${ }^{13}$ C NMR $\left(100.5 \mathrm{MHz}, \mathrm{CDCl}_{3}\right) \delta 159.6,145.2,139.1,135.8,134.1,130.0,129.8,128.2,127.8$, $127.5,127.3,120.2,112.4,105.8,94.2,85.2,55.4,21.7$.

IR (ATR) 2166, 1621, 1337, 1238, 1163, 1084, 1028, 939, 872, 813, 770, 716, $666 \mathrm{~cm}^{-1}$.

HRMS (EI-TOF) $m / z$ : [M] $]^{+}$Calcd for $\mathrm{C}_{20} \mathrm{H}_{16} \mathrm{O}_{3} \mathrm{~S} 336.0820$; Found 336.0834.

\section{1-Methyl-2-(tosylethynyl)benzene (2e)}<smiles>Cc1ccccc1C#CSOOS(=O)(=O)O</smiles>

$456.8 \mathrm{mg}, 84 \%$ yield, colorless solid. Mp $60.6-61.2^{\circ} \mathrm{C}$.

Rf: 0.39 (EtOAc/Hexane 2:8).

${ }^{1}$ H NMR $\left(400 \mathrm{MHz}, \mathrm{CDCl}_{3}\right) \delta$ 7.98-7.95 (m, 2H), $7.46(\mathrm{dd}, J=1.2,7.6 \mathrm{~Hz}, 1 \mathrm{H}), 7.39(\mathrm{~d}, J=7.6$ $\mathrm{Hz}, 2 \mathrm{H}), 7.35$ (ddd, $J=1.2,7.6,7.6 \mathrm{~Hz}, 2 \mathrm{H}), 7.22$ (d, $J=7.6 \mathrm{~Hz}, 1 \mathrm{H}), 7.17$ (dd, $J=7.6,7.6 \mathrm{~Hz}$, $1 \mathrm{H})$.

${ }^{13} \mathrm{C}$ NMR $\left(100.5 \mathrm{MHz}, \mathrm{CDCl}_{3}\right) \delta 145.3,142.4,139.2,133.0,131.5,130.0,129.9,127.4,125.9$, $117.8,92.6,89.2,21.8,20.4$.

IR (ATR) 2181, 1324, 1155, 1083, 866, 777, 762, $678 \mathrm{~cm}^{-1}$.

HRMS (EI-TOF) $m / z$ : [M] $]^{+}$Calcd for $\mathrm{C}_{16} \mathrm{H}_{14} \mathrm{O}_{2} \mathrm{~S} 270.0715$; Found 270.0702 . 
1-Methyl-3-(tosylethynyl)benzene (2f)<smiles></smiles>

389.3mg, 72\% yield, colorless solid. Mp 79.5-80.4 ${ }^{\circ} \mathrm{C}$

$\mathrm{R}_{\mathrm{f}}: 0.37$ (EtOAc/Hexane 2:8).

${ }^{1} \mathbf{H}$ NMR $\left(400 \mathrm{MHz}, \mathrm{CDCl}_{3}\right) \delta 7.95(\mathrm{~d}, J=8.8 \mathrm{~Hz}, 2 \mathrm{H}), 7.39(\mathrm{~d}, J=8.8 \mathrm{~Hz}, 2 \mathrm{H}), 7.35-7.31(\mathrm{~m}, 2 \mathrm{H})$, 7.29-7.22 (m, 2H).

${ }^{13}$ C NMR $\left(100.5 \mathrm{MHz}, \mathrm{CDCl}_{3}\right) \delta 145.3,139.0,138.6,133.1,132.4,130.0,129.8,128.5,127.5$, $117.7,93.4,85.2,21.7,21.1$.

IR (ATR) 2925, 2171, 1593, 1336, 1301, 1161, 1084, 932, 787, 765, 687, $675 \mathrm{~cm}^{-1}$.

HRMS (EI-TOF) $m / z$ : [M] ${ }^{+}$Calcd for $\mathrm{C}_{16} \mathrm{H}_{14} \mathrm{O}_{2} \mathrm{~S} 270.0715$; Found 270.0702.

\section{Characterization of products}

1-(4-Bromophenyl)-3-phenylprop-2-yn-1-ol (3aa)<smiles>OC(C#Cc1ccccc1)c1ccc(Br)cc1</smiles>

24.4. $\mathrm{mg}, 85 \%$ yield, colorless solid.

Rf: 0.48 (EtOAc/Hexane 3:7).

${ }^{1}$ H NMR (400 MHz, $\left.\mathrm{CDCl}_{3}\right) \delta$ 7.55-7.46 (m, 6H), 7.35-7.33 (m, 3H), $5.66(\mathrm{~d}, J=6.0 \mathrm{~Hz}, 1 \mathrm{H}), 2.28$ $(\mathrm{d}, J=6.0 \mathrm{~Hz}, 1 \mathrm{H})$.

${ }^{13}$ C NMR (100.5 MHz, $\left.\mathrm{CDCl}_{3}\right) \delta 139.5,131.7,128.8,128.4,128.3,122.4,122.1,88.1,87.0,64.4$. Spectral data of 3 aa was consistent with the literature data. ${ }^{2}$

1-(4-Chlorophenyl)-3-phenylprop-2-yn-1-ol (3ba)<smiles>OC(C#Cc1ccccc1)c1ccc(Cl)cc1</smiles>

$17.3 \mathrm{mg}, 76 \%$ yield, colorless solid. 
Rf: 0.48 (EtOAc/Hexane 3:7).

${ }^{1} \mathbf{H}$ NMR $\left(400 \mathrm{MHz}, \mathrm{CDCl}_{3}\right) \delta 7.55(\mathrm{~d}, J=8.7 \mathrm{~Hz}, 2 \mathrm{H}), 7.47-7.45(\mathrm{~m}, 2 \mathrm{H}), 7.38-7.32(\mathrm{~m}, 5 \mathrm{H}), 5.67$ $(\mathrm{d}, J=6.0 \mathrm{~Hz}, 1 \mathrm{H}), 2.40(\mathrm{~d}, J=6.0 \mathrm{~Hz}, 1 \mathrm{H})$.

${ }^{13}$ C NMR (100.5 MHz, $\left.\mathrm{CDCl}_{3}\right) \delta$ 139.0, 134.2, 131.7, 128.8, 128.3, 128.1, 122.1, 88.2, 86.9, 64.4. Spectral data of $\mathbf{3 b a}$ was consistent with the literature data. ${ }^{10}$

\section{1-(4-Fluorophenyl)-3-phenylprop-2-yn-1-ol (3ca)}<smiles>OC(C#Cc1ccccc1)c1ccc(F)cc1</smiles>

$15.6 \mathrm{mg}, 69 \%$ yield, colorless oil.

$\mathrm{R}_{\mathrm{f}}: 0.45$ (EtOAc/Hexane 3:7).

${ }^{1} \mathbf{H}$ NMR $\left(400 \mathrm{MHz}, \mathrm{CDCl}_{3}\right) \delta$ 7.63-7.58 (m, 2H), 7.49-7.46 (m, 2H), 7.36-7.30 (m, 3H), 7.12-7.06 $(\mathrm{m}, 2 \mathrm{H}), 5.68(\mathrm{~d}, J=5.0 \mathrm{~Hz}, 1 \mathrm{H}), 2.32(\mathrm{~d}, J=6.0 \mathrm{~Hz}, 1 \mathrm{H})$.

${ }^{13}$ C NMR $\left(100.5 \mathrm{MHz}, \mathrm{CDCl}_{3}\right) \delta 162.7(\mathrm{~d}, J=247 \mathrm{~Hz}), 136.4,131.7,128.7,128.7(\mathrm{~d}, J=8.6 \mathrm{~Hz})$, $128.3,122.2,115.5(\mathrm{~d}, J=22.1 \mathrm{~Hz}), 88.4,86.9,64.4$.

Spectral data of 3 ca was consistent with the literature data. ${ }^{11}$

\section{4-(1-Hydroxy-3-phenylprop-2-yn-1-yl)benzonitrile (3da)}<smiles>N#Cc1ccc(C(O)C#Cc2ccccc2)cc1</smiles>

$11.2 \mathrm{mg}, 48 \%$ yield, colorless oil.

$\mathrm{R}_{\mathrm{f}}$ 0.51 (EtOAc/Hexane 3:7).

${ }^{1}$ H NMR $\left(400 \mathrm{MHz}, \mathrm{CDCl}_{3}\right) \delta$ 7.76-7.69 (m, 4H), 7.48-7.45 (m, 2H), 7.39-7.30 (m, 3H), $5.75(\mathrm{~d}, J$ $=5.6 \mathrm{~Hz}, 1 \mathrm{H}), 2.40(\mathrm{~d}, J=5.6 \mathrm{~Hz}, 1 \mathrm{H})$.

${ }^{13}$ C NMR $\left(100.5 \mathrm{MHz}, \mathrm{CDCl}_{3}\right) \delta 145.44,132.47,131.76,129.03,128.42,127.28,121.73,118.65$, $112.14,87.56,87.36,64.25$.

IR (ATR) 3425, 2229, 2196, 1643, 1489, 1406, 1312, 1285, 1207, 1030, 1013, 854, 755, $689 \mathrm{~cm}^{-1}$. HRMS (EI-TOF) $m / z$ : [M] $]^{+}$Calcd for $\mathrm{C}_{16} \mathrm{H}_{11} \mathrm{NO} 233.0841$; Found, 233.0839. 
<smiles>COc1ccc(C(O)C#CCc2ccccc2)cc1</smiles>

$4.8 \mathrm{mg}, 20 \%$ yield, colorless oil.

$\mathrm{R}_{\mathrm{f}}$ 0.74 (EtOAc/Hexane 1:1).

${ }^{1} \mathbf{H}$ NMR $\left(400 \mathrm{MHz}, \mathrm{CDCl}_{3}\right) \delta$ 7.53-7.57 (m, 2H), 7.45-7.49 (m, 2H), 7.29-7.35 (m, 3H), 6.91-6.95 $(\mathrm{m}, 2 \mathrm{H}), 5.65(\mathrm{~d}, J=5.6 \mathrm{~Hz}, 1 \mathrm{H}), 3.83(\mathrm{~s}, 3 \mathrm{H}), 2.23(\mathrm{~d}, J=5.6 \mathrm{~Hz}, 1 \mathrm{H})$.

${ }^{13}$ C NMR $\left(100.5 \mathrm{MHz}, \mathrm{CDCl}_{3}\right) \delta 159.7,132.9,131.7,128.6,128.3,128.2,114.0,88.8,86.5,64.7$, 55.3 .

Spectral data of 3ea was consistent with the literature data. ${ }^{12}$

\section{1-(2-Bromophenyl)-3-phenylprop-2-yn-1-ol (3fa)}<smiles>OC(C#Cc1ccccc1)c1ccccc1Br</smiles>

$16.1 \mathrm{mg}, 56 \%$ yield, colorless oil.

Rf: 0.51 (EtOAc/Hexane 3:7).

${ }^{1}$ H NMR $\left(400 \mathrm{MHz}, \mathrm{CDCl}_{3}\right) \delta 7.85(\mathrm{dd}, J=7.6,1.6 \mathrm{~Hz}, 1 \mathrm{H}), 7.59(\mathrm{dd}, J=8.2,0.9 \mathrm{~Hz}, 1 \mathrm{H}), 7.49-$ $7.46(\mathrm{~m}, 2 \mathrm{H}), 7.38(\mathrm{t}, J=7.2 \mathrm{~Hz}, 1 \mathrm{H}), 7.33-7.30(\mathrm{~m}, 3 \mathrm{H}), 7.21(\mathrm{td}, J=7.7,1.5 \mathrm{~Hz}, 1 \mathrm{H}), 6.02(\mathrm{~d}, J=$ $5.2 \mathrm{~Hz}, 1 \mathrm{H}), 2.63(\mathrm{~d}, J=5.2 \mathrm{~Hz}, 1 \mathrm{H})$.

${ }^{13} \mathrm{C}$ NMR $\left(100.5 \mathrm{MHz}, \mathrm{CDCl}_{3}\right) \delta 139.4,133.0,131.7,130.0,128.7,128.3,127.9,122.8,122.2$, 87.6, 86.8, 64.7.

HRMS (ESI-FT) $m / z$ : [M+Na $]^{+}$Calcd for $\mathrm{C}_{15} \mathrm{H}_{11} \mathrm{BrONa} 308.9886$; Found 308.9884 .

Spectral data of $\mathbf{3 f a}$ was consistent with the literature data. ${ }^{11}$

\section{3-Phenyl-1-(2-(trifluoromethyl)phenyl)prop-2-yn-1-ol (3ga)}<smiles>OC(C#Cc1ccccc1)c1ccccc1C(F)(F)F</smiles>

$14.3 \mathrm{mg}, 52 \%$ yield, colorless oil.

Rf: 0.48 (EtOAc/Hexane 3:7). 
${ }^{1}$ H NMR $\left(400 \mathrm{MHz}, \mathrm{CDCl}_{3}\right) \delta 8.09(\mathrm{~d}, J=7.8 \mathrm{~Hz}, 2 \mathrm{H}), 7.69-7.63(\mathrm{~m}, 2 \mathrm{H}), 7.48-7.42(\mathrm{~m}, 3 \mathrm{H})$, 7.33-7.28 (m, 3H), $6.08(\mathrm{~s}, 1 \mathrm{H}), 2.48$ (brs, 1H).

${ }^{13}$ C NMR $\left(100.5 \mathrm{MHz}, \mathrm{CDCl}_{3}\right) \delta 139.2,132.6,131.7,129.1,128.7,128.6,128.3,127.2(\mathrm{q}, J=31.0$ $\mathrm{Hz}), 125.8$ (q, $J=5.7 \mathrm{~Hz}), 124.2(\mathrm{q}, J=274.1 \mathrm{~Hz}), 122.1,88.1,86.7,61.0(\mathrm{~d}, J=1.9 \mathrm{~Hz})$.

Spectral data of 3 ga was consistent with the literature data. ${ }^{13}$

\section{3-Phenyl-1-(o-tolyl)prop-2-yn-1-ol (3ha)}<smiles>Nc1ccccc1C(O)C#Cc1ccccc1</smiles>

$14.2 \mathrm{mg}, 64 \%$ yield, colorless oil.

Rf: 0.59 (EtOAc/Hexane 3:7).

${ }^{1} \mathbf{H}$ NMR $\left(400 \mathrm{MHz}, \mathrm{CDCl}_{3}\right) \delta$ 7.76-7.71 (m, 1H), 7.48-7.45 (m, 2H), 7.32-7.30 (m, 3H), 7.27-7.19 $(\mathrm{m}, 3 \mathrm{H}), 5.84(\mathrm{~d}, J=6.0 \mathrm{~Hz}, 1 \mathrm{H}), 2.50(\mathrm{~s}, 3 \mathrm{H}), 2.20(\mathrm{~d}, J=6.0 \mathrm{~Hz}, 1 \mathrm{H})$.

${ }^{13}$ C NMR $\left(100.5 \mathrm{MHz}, \mathrm{CDCl}_{3}\right) \delta 138.3,136.0,131.7,130.8,128.5,128.5,128.3,126.6,126.2$, $122.5,88.4,86.5,63.0,19.0$.

IR (ATR) 3338, 3022, 1721, 1489, 1443, 1259, 1215, 1033, 1016, 998, 961, 753, $690 \mathrm{~cm}^{-1}$.

HRMS (APCI-FT) $m / z$ : [M+H] $]^{+}$Calcd for $\mathrm{C}_{16} \mathrm{H}_{15} \mathrm{O} 223.1118$; Found, 223.1117 .

\section{1-(3,5-Dibromophenyl)-3-phenylprop-2-yn-1-ol (3ia)}<smiles>OC(C#Cc1ccccc1)c1cc(Br)cc(Br)c1</smiles>

$23.3 \mathrm{mg}, 64 \%$ yield, colorless solid. Mp 101.5-102.3 ${ }^{\circ} \mathrm{C}$.

Rf: 0.59 (EtOAc/Hexane 3:7).

${ }^{1} \mathbf{H}$ NMR (400 MHz, $\left.\mathrm{CDCl}_{3}\right) \delta 7.69(\mathrm{~d}, J=1.8 \mathrm{~Hz}, 2 \mathrm{H}), 7.64(\mathrm{t}, J=1.8 \mathrm{~Hz}, 1 \mathrm{H}), 7.49-7.46(\mathrm{~m}, 2 \mathrm{H})$, 7.37-7.34 (m, 3H), $5.64(\mathrm{~d}, J=6.0 \mathrm{~Hz}, 1 \mathrm{H}), 2.43(\mathrm{~d}, J=6.0 \mathrm{~Hz}, 1 \mathrm{H})$.

${ }^{13}$ C NMR $\left(100.5 \mathrm{MHz}, \mathrm{CDCl}_{3}\right) \delta 144.2,133.9,131.8,129.0,128.5,128.4,123.1,121.7,87.5,87.2$, 63.7.

IR (ATR) 3311, 1585, 1558, 1490, 1418, 1304, 1183, 1154, 1104, 1032, 975, 856, 753, 735, 689 $\mathrm{cm}^{-1}$.

HRMS (ESI-FT) $m / z$ : [M-H] $]^{-}$calcd for $\mathrm{C}_{15} \mathrm{H}_{9} \mathrm{Br}_{2} \mathrm{O} 362.9025$; Found 362.9025. 
1-(2,6-Dimethylphenyl)-3-phenylprop-2-yn-1-ol (3ja)<smiles></smiles>

$13.9 \mathrm{mg}, 59 \%$ yield, colorless solid. Mp 58.5-59.8 ${ }^{\circ} \mathrm{C}$.

$\mathrm{R}_{\mathrm{f}}: 0.49$ (EtOAc/Hexane 3:7).

${ }^{1} \mathbf{H}$ NMR $\left(400 \mathrm{MHz}, \mathrm{CDCl}_{3}\right) \delta$ 7.43-7.41 (m, 2H), 7.30-7.29 (m, 3H), 7.12 (dd, $\left.J=8.0,7.6 \mathrm{~Hz}, 1 \mathrm{H}\right)$, $7.05(\mathrm{~d}, J=7.6 \mathrm{~Hz}, 1 \mathrm{H}), 2.60(\mathrm{~s}, 1 \mathrm{H}), 2.15(\mathrm{~s}, 1 \mathrm{H})$.

${ }^{13}$ C NMR $\left(100.5 \mathrm{MHz}, \mathrm{CDCl}_{3}\right) \delta 136.7,136.3,131.6,129.3,128.4,128.2,128.2,122.7,88.5,85.8$, $60.9,20.4$

Spectral data of $\mathbf{3} \mathbf{j a}$ was consistent with the literature data. ${ }^{14}$

\section{1-(2,6-Dichlorophenyl)-3-phenylprop-2-yn-1-ol (3ka)}<smiles>OC(C#CC#P)c1c(Cl)cccc1Cl</smiles>

3ka

$20.7 \mathrm{mg}, 75 \%$ yield, colorless oil.

$\mathrm{R}_{\mathrm{f}}$ : 0.55 (EtOAc/Hexane 3:7).

${ }^{1}$ H NMR (400 MHz, $\left.\mathrm{CDCl}_{3}\right) \delta$ 7.46-7.43 (m, 2H), $7.35(\mathrm{~d}, J=7.6 \mathrm{~Hz}, 2 \mathrm{H}), 7.32-7.26(\mathrm{~m}, 3 \mathrm{H}), 7.20$ $(\mathrm{dd}, J=8.8,7.6 \mathrm{~Hz}, 1 \mathrm{H}), 6.40(\mathrm{~d}, J=10.1 \mathrm{~Hz}, 1 \mathrm{H}), 3.40(\mathrm{~d}, J=10.1 \mathrm{~Hz}, 1 \mathrm{H})$.

${ }^{13} \mathrm{C}$ NMR $\left(100.5 \mathrm{MHz}, \mathrm{CDCl}_{3}\right) \delta 135.5,134.4,131.8,129.7,129.3,128.7,128.3,122.3,86.6,86.2$, 61.4 .

HRMS (APCI-FT) $m / z$ : $[\mathrm{M}+\mathrm{H}]^{+}$Calcd for $\mathrm{C}_{15} \mathrm{H}_{11} \mathrm{Cl}_{2} \mathrm{O}$ 277.0182; Found 277.0189.

Spectral data of $\mathbf{3 k a}$ was consistent with the literature data. ${ }^{15}$

1-(Naphthalen-1-yl)-3-phenylprop-2-yn-1-ol (3la)<smiles>OC(C#CC#Cc1ccccc1)c1cccc2ccccc12</smiles>

$11.2 \mathrm{mg}, 43 \%$ yield, yellow oil.

$\mathrm{R}_{\mathrm{f}}: 0.45$ (EtOAc/Hexane 3:7). 
${ }^{1} \mathbf{H}$ NMR $\left(400 \mathrm{MHz}, \mathrm{CDCl}_{3}\right) \delta 8.38(\mathrm{~d}, J=8.4 \mathrm{~Hz}, 1 \mathrm{H}), 7.94-7.85(\mathrm{~m}, 3 \mathrm{H}), 7.58(\mathrm{t}, J=7.1 \mathrm{~Hz}, 1 \mathrm{H})$, 7.54-7.45 (m, 4H), 7.33-7.29 (m, 3H), 6.35 (d, $J=4.4 \mathrm{~Hz}, 1 \mathrm{H}), 2.48$ (d, $J=4.4 \mathrm{~Hz}, 1 \mathrm{H})$.

${ }^{13}$ C NMR $\left(100.5 \mathrm{MHz}, \mathrm{CDCl}_{3}\right) \delta 135.6,134.0,131.7,130.6,129.4,128.7,128.6,128.3,126.5$, $125.9,125.2,124.7,123.9,122.4,88.4,87.3,63.4$.

Spectral data of 3la was consistent with the literature data. ${ }^{12}$

\section{3-Phenyl-1-(thiophen-2-yl)prop-2-yn-1-ol (3ma)}

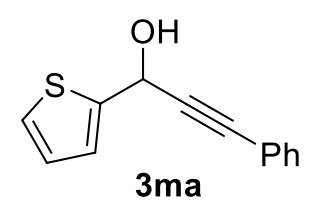

$12.4 \mathrm{mg}, 58 \%$ yield, colorless oil.

Rf: 0.45 (EtOAc/Hexane 3:7).

${ }^{1} \mathbf{H}$ NMR $\left(400 \mathrm{MHz}, \mathrm{CDCl}_{3}\right) \delta$ 7.52-7.47 (m, 2H), 7.37-7.31 (m, 4H), 7.03-6.99 (m, 1H), 5.89 (d, $J$ $=6.8 \mathrm{~Hz}, 1 \mathrm{H}), 2.42(\mathrm{~d}, J=6.8 \mathrm{~Hz}, 1 \mathrm{H})$.

${ }^{13}$ C NMR $\left(100.5 \mathrm{MHz}, \mathrm{CDCl}_{3}\right) \delta 144.6,131.8,128.8,128.3,126.8,126.2,125.7,122.1,87.9,86.1$, 60.8 .

Spectral data of $\mathbf{3 m a}$ was consistent with the literature data. ${ }^{12}$

Methyl 4-(1-hydroxy-3-phenylprop-2-yn-1-yl)benzoate (3na)<smiles>COC(=O)c1ccc(C(O)C#Cc2ccccc2)cc1</smiles>

$16.2 \mathrm{mg}, 61 \%$ yield, colorless oil.

Rf: 0.69 (EtOAc/Hexane 1:1).

${ }^{1} \mathbf{H}$ NMR $\left(400 \mathrm{MHz}, \mathrm{CDCl}_{3}\right) \delta 8.07(\mathrm{~d}, J=8.0 \mathrm{~Hz}, 2 \mathrm{H}), 7.68(\mathrm{~d}, J=8.0 \mathrm{~Hz}, 2 \mathrm{H}), 7.48-7.45(\mathrm{~m}, 2 \mathrm{H})$, 7.35-7.30 (m, 3H), $5.74(\mathrm{~d}, J=5.0 \mathrm{~Hz}, 1 \mathrm{H}), 3.92(\mathrm{~s}, 3 \mathrm{H}), 2.67-2.58(\mathrm{~m}, 1 \mathrm{H})$.

${ }^{13}$ C NMR $\left(100.5 \mathrm{MHz}, \mathrm{CDCl}_{3}\right) \delta 166.8,145.4,131.7,130.0,129.9,128.8,128.3,126.6,122.0$, 88.0, 87.1, 64.5, 52.2.

Spectral data of 3 na was consistent with the literature data. ${ }^{16}$ 
1-(4-(1-Hydroxy-3-phenylprop-2-yn-1-yl)phenyl)ethan-1-one (30a)<smiles>COc1ccc(C#CC(O)c2ccc(C(C)=O)cc2)cc1</smiles>

$8.9 \mathrm{mg}, 32 \%$, yellow oil.

$\mathrm{R}_{\mathrm{f}}: 0.58$ (EtOAc/Hexane 1:1).

${ }^{1} \mathbf{H}$ NMR $\left(400 \mathrm{MHz}, \mathrm{CDCl}_{3}\right) \delta 8.00(\mathrm{~d}, J=8.4 \mathrm{~Hz}, 2 \mathrm{H}), 7.71(\mathrm{~d}, J=8.8 \mathrm{~Hz}, 2 \mathrm{H}), 7.39-7.42(\mathrm{~m}, 2 \mathrm{H})$, 6.83-6.87 (m, 2H), 5.74 (brs, 1H), $3.82(\mathrm{~s}, 3 \mathrm{H}), 2.62$ (s, 3H), 2.46 (brs, $1 \mathrm{H})$.

${ }^{13}$ C NMR $\left(100.5 \mathrm{MHz}, \mathrm{CDCl}_{3}\right) \delta 197.8,160.0,145.7,136.9,133.3,128.7,126.8,114.0,114.0$, 87.2, 86.7, 64.6, 55.3, 26.7 .

IR (ATR) 3395, 2839, 1677, 1606, 1508, 1290, 1266, 1246, 1172, 1030, 958, 831, $750 \mathrm{~cm}^{-1}$.

HRMS (ESI-FT) $m / z$ : [M+Na $]^{+}$Calcd for $\mathrm{C}_{18} \mathrm{H}_{16} \mathrm{O}_{3} \mathrm{Na}$ 303.0992; Found 303.0996.

\section{4-(1-Hydroxy-3-phenylprop-2-yn-1-yl)benzoic acid (3pa)}<smiles>O=C(O)c1ccc(C(O)C#CPc2ccccc2)cc1</smiles>

$24.2 \mathrm{mg}$, 96\% yield, colorless solid. Mp $241.2^{\circ} \mathrm{C} \mathrm{dec}$.

$\mathrm{R}_{\mathrm{f}}: 0.41\left(\mathrm{MeOH} / \mathrm{CHCl}_{3}\right.$ 1:4).

${ }^{1} \mathbf{H}$ NMR (400 MHz, CD $\left.{ }_{3} \mathrm{OD}\right) \delta 8.03(\mathrm{~d}, J=7.6 \mathrm{~Hz}, 2 \mathrm{H}), 7.64(\mathrm{~d}, J=7.6 \mathrm{~Hz}, 2 \mathrm{H}), 7.48-7.42(\mathrm{~m}$, 2H), 7.37-7.31 (m, 3H), $5.68(\mathrm{~s}, 1 \mathrm{H})$.

${ }^{13} \mathrm{C}$ NMR $\left(100.5 \mathrm{MHz}, \mathrm{CD}_{3} \mathrm{OD}\right) \delta 172.8,146.4,135.2,132.6,130.8,129.6,129.5,127.4,124.0$, $90.2,86.8,65.0$.

IR (ATR) 3310, 2925, 2525, 1691, 1595, 1542, 1408, 1278, 1031, 1016, 998, 752, $689 \mathrm{~cm}^{-1}$.

HRMS (ESI-FT) $m / z$ : [M-H] $]^{-}$Calcd for $\mathrm{C}_{16} \mathrm{H}_{11} \mathrm{O}_{3}$ 251.0713; Found 251.0717.

3-(4-(1-Hydroxy-3-phenylprop-2-yn-1-yl)phenyl)propanal (3qa)<smiles>COc1ccc(C#CC(O)c2ccc(CCC=O)cc2)cc1</smiles>

$11.5 \mathrm{mg}, 39 \%$ yield, colorless oil.

$\mathrm{R}_{\mathrm{f}}: 0.56$ (EtOAc/Hexane 1:1). 
${ }^{1}$ H NMR (400 MHz, $\left.\mathrm{CDCl}_{3}\right) \delta 9.83$ (brs, 1H), $7.54(\mathrm{~d}, J=8.0 \mathrm{~Hz}, 2 \mathrm{H}), 7.40(\mathrm{~d}, J=8.4 \mathrm{~Hz}, 2 \mathrm{H})$, $7.23(\mathrm{~d}, J=8.0 \mathrm{~Hz}, 2 \mathrm{H}), 6.84(\mathrm{~d}, J=8.4 \mathrm{~Hz}, 2 \mathrm{H}), 5.66$ (brs, $1 \mathrm{H}), 3.82(\mathrm{~s}, 3 \mathrm{H}), 2.98$ (t, $J=8.0 \mathrm{~Hz}$, 2H), 2.79 (t, $J=8.0 \mathrm{~Hz}, 2 \mathrm{H}), 2.24$ (brs, $1 \mathrm{H})$.

${ }^{13}$ C NMR $\left(100.5 \mathrm{MHz}, \mathrm{CDCl}_{3}\right) \delta 201.4,159.8,140.6,138.9,133.2,128.6,127.0,114.4,113.9$, $87.3,86.6,64.9,55.3,45.2,27.8$.

IR (ATR) 3406, 2926, 1718, 1605, 1509, 1291, 1248, 1172, 1031, $832 \mathrm{~cm}^{-1}$.

HRMS (EI-TOF) $m / z$ : [M] ${ }^{+}$Calcd for $\mathrm{C}_{19} \mathrm{H}_{18} \mathrm{O}_{3}$ 294.1256; Found 294.1265.

1-(4-Bromophenyl)-3-(4-methoxyphenyl)prop-2-yn-1-ol (3ab)<smiles>COc1ccc(C#CC(O)c2ccc(Br)cc2)cc1</smiles>

$0.1 \mathrm{mmol}$ scale: $34.5 \mathrm{mg}, 98 \%$ yield, yellow oil.

$1.0 \mathrm{mmol}$ scale: $275 \mathrm{mg}, 87 \%$ yield, yellow oil.

Rf: 0.38 (EtOAc/Hexane 3:7).

${ }^{1} \mathbf{H}$ NMR $\left(400 \mathrm{MHz}, \mathrm{CDCl}_{3}\right) \delta 7.53(\mathrm{~d}, J=8.0 \mathrm{~Hz}, 2 \mathrm{H}), 7.48(\mathrm{~d}, J=8.0 \mathrm{~Hz}, 2 \mathrm{H}), 7.40(\mathrm{~d}, J=7.8$ $\mathrm{Hz}, 2 \mathrm{H}), 6.85$ (d, $J=7.8 \mathrm{~Hz}, 2 \mathrm{H}), 5.64$ (s, 1H), 3.82 (s, 3H), 2.30 (brs, 1H).

${ }^{13}$ C NMR $\left(100.5 \mathrm{MHz}, \mathrm{CDCl}_{3}\right) \delta 159.9,139.8,133.2,131.7,128.4,122.3,114.1,114.0,87.0,86.8$, 64.5, 55.3.

IR (ATR) 3360, 2837, 2170, 1605, 1508, 1484, 1290, 1246, 1172, 1030, 1010, 962, $830 \mathrm{~cm}^{-1}$.

HRMS (APCI-FT) $m / z$ : [M+H] $]^{+}$Calcd for $\mathrm{C}_{16} \mathrm{H}_{14} \mathrm{BrO}_{2}$ 317.0172; Found 317.0178.

\section{1,3-Bis(4-bromophenyl)prop-2-yn-1-ol (3ac)}<smiles>OC(C#Cc1ccc(Br)cc1)c1ccc(Br)cc1</smiles>

$7.0 \mathrm{mg}, 19 \%$ yield, colorless solid. Mp $98.8-99.5{ }^{\circ} \mathrm{C}$.

Rf: 0.49 (EtOAc/Hexane 3:7).

${ }^{1} \mathbf{H}$ NMR $\left(400 \mathrm{MHz}, \mathrm{CDCl}_{3}\right) \delta$ 7.56-7.51 (m, 2H), 7.50-7.44 (m, 4H), 7.34-7.29 (m, 2H), 5.64 (d, $J$ $=6.0 \mathrm{~Hz}, 1 \mathrm{H}), 2.32(\mathrm{brs}, 1 \mathrm{H})$.

${ }^{13}$ C NMR $\left(100.5 \mathrm{MHz}, \mathrm{CDCl}_{3}\right) \delta 139.3,133.2,131.8,131.6,128.4,123.1,122.5,121.0,89.2,85.9$, 64.4 .

Spectral data of 3ac was consistent with the literature data. ${ }^{17}$ 
<smiles>COc1ccc2cc(C#CC(O)c3ccc(Br)cc3)ccc2c1</smiles>

$12.4 \mathrm{mg}, 34 \%$ yield, colorless solid. Mp $154.5-156.1^{\circ} \mathrm{C}$.

$\mathrm{R}_{\mathrm{f}}: 0.69$ (EtOAc/Hexane 1:1).

${ }^{1} \mathbf{H}$ NMR $\left(400 \mathrm{MHz}, \mathrm{CDCl}_{3}\right) \delta 7.91(\mathrm{~s}, 1 \mathrm{H}), 7.69$ (d, $\left.J=4.8 \mathrm{~Hz}, 1 \mathrm{H}\right), 7.67$ (d, J=4.8 Hz, 1H), 7.56$7.51(\mathrm{~m}, 4 \mathrm{H}), 7.46(\mathrm{dd}, J=8.8,1.6 \mathrm{~Hz}, 1 \mathrm{H}), 7.16(\mathrm{dd}, J=9.2,2.4 \mathrm{~Hz}, 1 \mathrm{H}), 7.10(\mathrm{~d}, J=2.4 \mathrm{~Hz}, 1 \mathrm{H})$, $5.69(\mathrm{~d}, J=6.0 \mathrm{~Hz}, 1 \mathrm{H}), 3.92(\mathrm{~s}, 3 \mathrm{H}), 2.39$ (d, $J=6.0 \mathrm{~Hz}, 1 \mathrm{H})$.

${ }^{13}$ C NMR $\left(100.5 \mathrm{MHz}, \mathrm{CDCl}_{3}\right) \delta 158.6,139.8,134.5,131.9,131.8,129.4,129.0,128.6,128.4$, $127.0,122.5,119.7,117.0,105.9,87.8,87.6,64.7,55.5$.

IR (ATR) 3512, 3006, 1626, 1600, 1484, 1389, 1266, 1246, 1200, 1165, 1023, 981, 904, 862, 820, $779,665 \mathrm{~cm}^{-1}$.

HRMS (APCI-FT) $m / z$ : [M+H] $]^{+}$Calcd for $\mathrm{C}_{20} \mathrm{H}_{16} \mathrm{BrO}_{2}$ 367.0329; Found 367.0337.

\section{1-(4-Bromophenyl)-3-(o-tolyl)prop-2-yn-1-ol (3ae)}<smiles>Cc1ccccc1C#CC(O)c1ccc(Br)cc1</smiles>

$23.7 \mathrm{mg}, 79 \%$ yield, colorless oil.

Rf: 0.49 (EtOAc/Hexane 3:7).

${ }^{1}$ H NMR $\left(400 \mathrm{MHz}, \mathrm{CDCl}_{3}\right) \delta$ 7.53-7.48 (m, 4H), 7.45-7.42 (m, 1H), 7.25-7.19 (m, 2H), 7.19-7.12 $(\mathrm{m}, 1 \mathrm{H}), 5.68(\mathrm{~s}, 1 \mathrm{H}), 2.45-2.42(\mathrm{~m}, 4 \mathrm{H})$.

${ }^{13}$ C NMR (100.5 MHz, $\left.\mathrm{CDCl}_{3}\right) \delta 140.3,139.7,132.1,131.7,129.5,128.8,128.4,125.6,122.3$, $121.8,92.0,85.9,64.5,20.7$.

IR (ATR) 3324, 1710, 1591, 1484, 1400, 1069, 1010, 961, 815, 753, $715 \mathrm{~cm}^{-1}$.

HRMS (ESI-FT) $m / z$ : [M-H] $]^{-}$Calcd for $\mathrm{C}_{16} \mathrm{H}_{12} \mathrm{BrO} 299.0077$; Found 299.0077. 
<smiles>Cc1cccc(C#CC(O)c2ccc(Br)cc2)c1</smiles>

$22.5 \mathrm{mg}, 91 \%$ yield, colorless oil.

$\mathrm{R}_{\mathrm{f}}$ 0.56 (EtOAc/Hexane 3:7).

${ }^{1} \mathbf{H}$ NMR $\left(400 \mathrm{MHz}, \mathrm{CDCl}_{3}\right) \delta 7.52(\mathrm{~d}, J=8.4 \mathrm{~Hz}, 2 \mathrm{H}), 7.48(\mathrm{~d}, J=8.4 \mathrm{~Hz}, 2 \mathrm{H}), 7.30-7.25(\mathrm{~m}, 2 \mathrm{H})$, $7.21(\mathrm{dd}, J=7.3,7.3 \mathrm{~Hz}, 1 \mathrm{H}), 7.15(\mathrm{~d}, J=7.3 \mathrm{~Hz}, 1 \mathrm{H}), 5.64(\mathrm{~s}, 1 \mathrm{H}), 2.46(\mathrm{~s}, 1 \mathrm{H}), 2.32(\mathrm{~s}, 3 \mathrm{H})$.

${ }^{13}$ C NMR $\left(100.5 \mathrm{MHz}, \mathrm{CDCl}_{3}\right) \delta 139.6,138.1,132.3,131.7,129.7,128.8,128.4,128.2,122.4$, 121.9, 87.7, 87.1, 64.4, 21.2.

IR (ATR) 3324, 2921, 1601, 1579, 1484, 1400, 1069, 1033, 1011, 971, 783, 753, 724, $690 \mathrm{~cm}^{-1}$. HRMS (ESI-FT) $m / z$ : [M-H] $]^{-}$Calcd for $\mathrm{C}_{16} \mathrm{H}_{12} \mathrm{BrO} 299.0077$; Found 299.0077.

\section{1-(4-Bromophenyl)-3-(trimethylsilyl)prop-2-yn-1-ol (3ag)}<smiles>CC#CC(O)c1ccc(Br)cc1</smiles>

$10.5 \mathrm{mg}, 37 \%$ yield, colorless oil.

Rf: 0.63 (EtOAc/Hexane 3:7).

${ }^{1}$ H NMR (400 MHz, $\left.\mathrm{CDCl}_{3}\right) \delta$ 7.53-7.49 (m, 2H), 7.43-7.40 (m, 2H), $5.41(\mathrm{~d}, J=5.6 \mathrm{~Hz}, 1 \mathrm{H}), 2.23$ $(\mathrm{d}, J=5.6 \mathrm{~Hz}, 1 \mathrm{H}), 0.20(\mathrm{~s}, 9 \mathrm{H})$.

${ }^{13}$ C NMR (100.5 MHz, $\left.\mathrm{CDCl}_{3}\right) \delta 139.3,131.7,128.4,122.4,104.3,92.1,64.3,-0.3$. IR (ATR) 3316, 2960, 1717, 1485, 1405, 1250, 1070, 1037, 1013, 981, 839, 788, $738 \mathrm{~cm}^{-1}$. HRMS (EI-TOF) $m / z$ : [M] ${ }^{+}$Calcd for $\mathrm{C}_{12} \mathrm{H}_{15} \mathrm{BrOSi} 282.0076$; Found 282.0070. 


\section{References}

1) Schneider, L, M.; Schmiedel, V, M.; Pecchioli, T.; Lentz, D.; Merten, C.; Christmann, M. Org. Lett. 2017, 19, 2310-2313.

2) Zhu,H.-T.; Ji, K.-G.; Yang, F.; Wang, L.-J.; Zhao, S.-C.; Ali, S.; Liu,X.-Y.; Liang, Y.-M. Org. Lett. 2011, 13, 684-687.

3) Prier, C. K.; Rankic, D. A.; MacMillan, D. W. C. Chem. Rev. 2013, 113, 5322-5363.

4) Haga, M.-A.; Dodsworth, E. S.; Eryavec, G.; Seymour, P.; Lever, A. B. P. Inorg. Chem. 1985, 24, 1901-1906.

5) Srivastava, V.; Singh, P. P. RSC Adv. 2017, 7, 31377-31392.

6) Ishimatsu, R.; Matsunami, S.; Kasahara, T.; Mizuno, J.; Edura, T.; Adachi, C.; Nakano, K.; Imato, T. Angew. Chem. Int. Ed. 2014, 53, 6993-6996.

7) Lowry, M. S.; Goldsmith, J. I.; Slinker, J. D.; Rohl, R.; Pascal, R. A.; Malliaras, G. G.; Bernhard, S. Chem. Mater. 2005, 17, 5712-5719.

8) (a) Tarantino, K. T.; Liu, P.; Knowles, R. R. J. Am. Chem. Soc. 2013, 135, 10022-10025. (b) Nakajima, M.; Fava, E.; Loescher, S.; Jiang, Z.; Rueping, M. Angew. Chem. Int. Ed. 2015, 54, 8828-8832. (c) Qi, L.; Chen, Y. Angew. Chem. Int. Ed. 2016, 55, 13312-13315.

9) (a) Meesin, J.; Katrun, P.; Pareseecharoen, C.; Pohmakotr, M.; Reutrakul, V.; Soorukram, D.; Kuhakarn, C. J. Org. Chem. 2016, 81, 2744-2752. (b) Gnägi, L.; Martz, S, V.; Meyer, D.; Schärer, R, M.; Renaud, P. Chem. Eur. J. 2019, 25, 11646-11649.

10) Sharma, S. K.; Gupta, S.; Saifuddin, M.; Mandadapu, A. K.; Agarwal, P. K.; Gauniyal, H. M.; Kundu, B. Tetrahedron Lett. 2011, 52, 65-68.

11) Sakai, N.; Kanada, R.; Hirasawa, M.; Konakahara, T. Tetrahedron 2005, 61, 9298-9304.

12) Downey,C. W.; Mahoney, B. D.; Lipari, V. R. J. Org. Chem. 2009, 74, 2904-2906.

13) Takita, R.; Fukuta, Y.; Tsuji, R.; Ohshima, T.; Shibasaki, M. Org. Chem. 2005, 7, 1363-1366.

14) Wadhwa, K.; Chintareddy, V. R.; Verkade, J. G. J. Org. Chem. 2009, 74, 6681-6690.

15) Liu, L.; Pu, L. Tetrahedron 2004, 60, 7427-7430.

16) Wilson, E. E.; Oliver, A. G.; Hughes, R. P.; Ashfeld, B. L. Organometallics 2011, 30, 52145221.

17) Janßen, C. E.; Krause, N. Eur. J. Org. Chem. 2005, 2322-2329. 


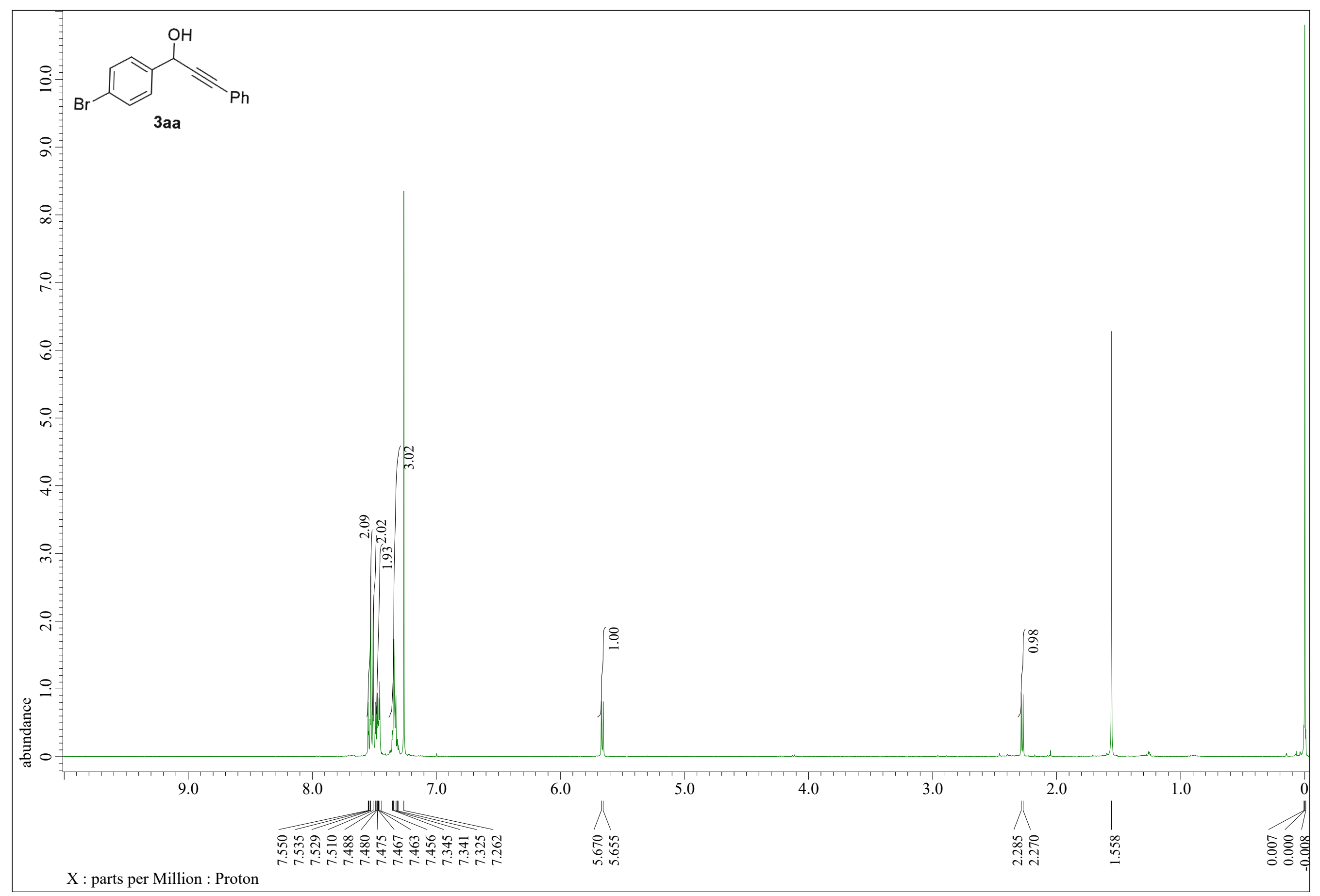


$100.5 \mathrm{MHz}, \mathrm{CDCl}_{3}$

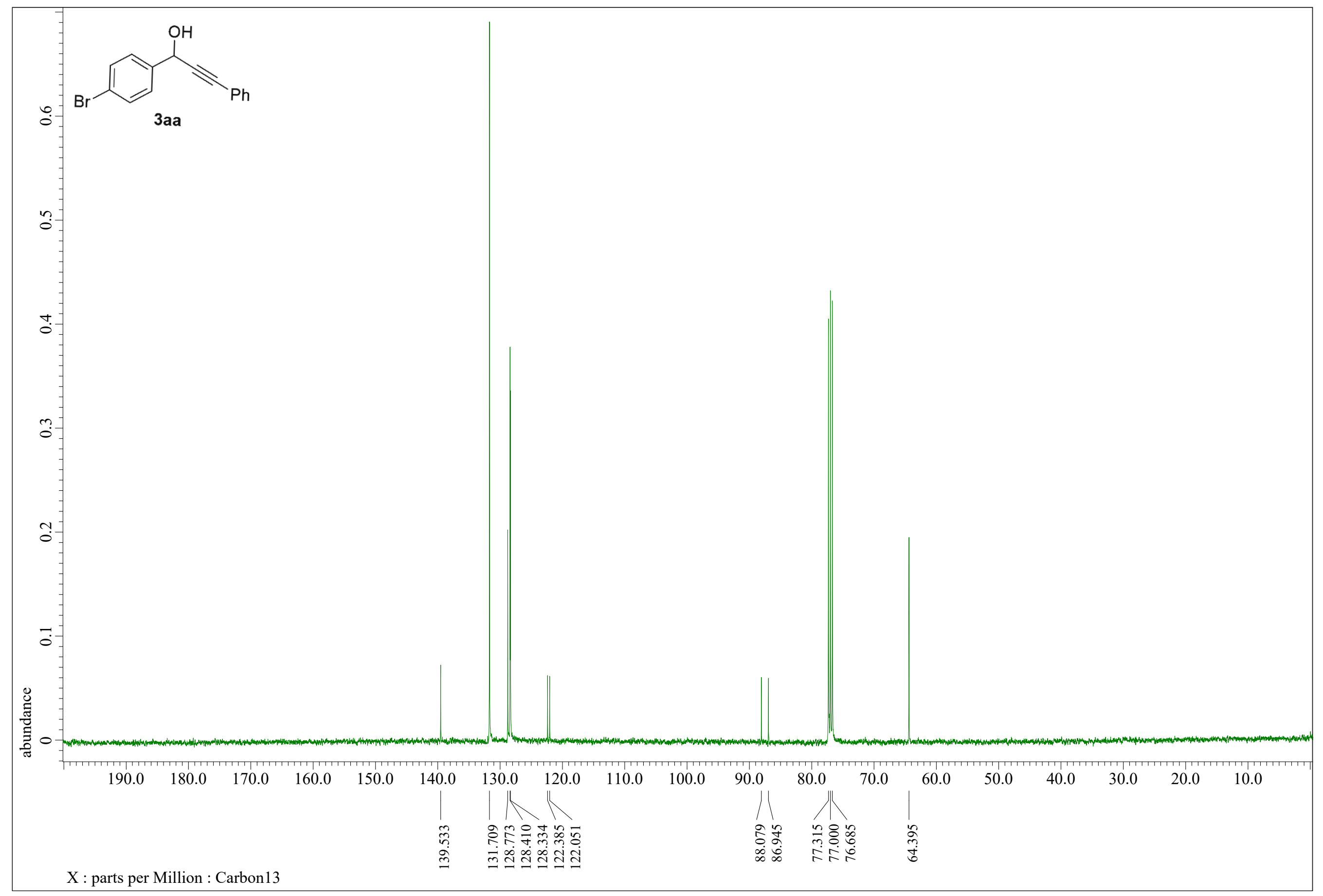


$400 \mathrm{MHz}, \mathrm{CDCl}_{3}$

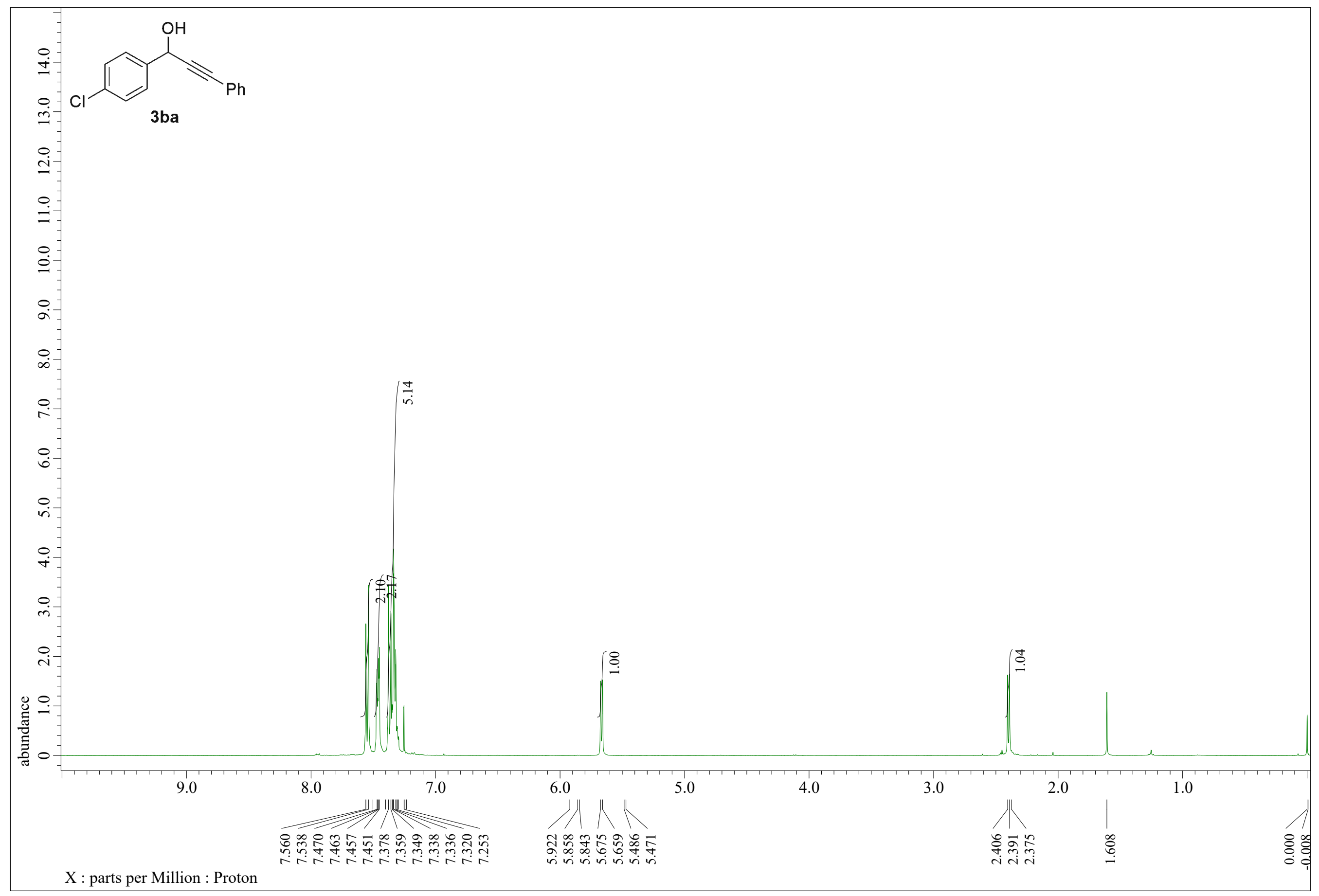


$100.5 \mathrm{MHz}, \mathrm{CDCl}_{3}$

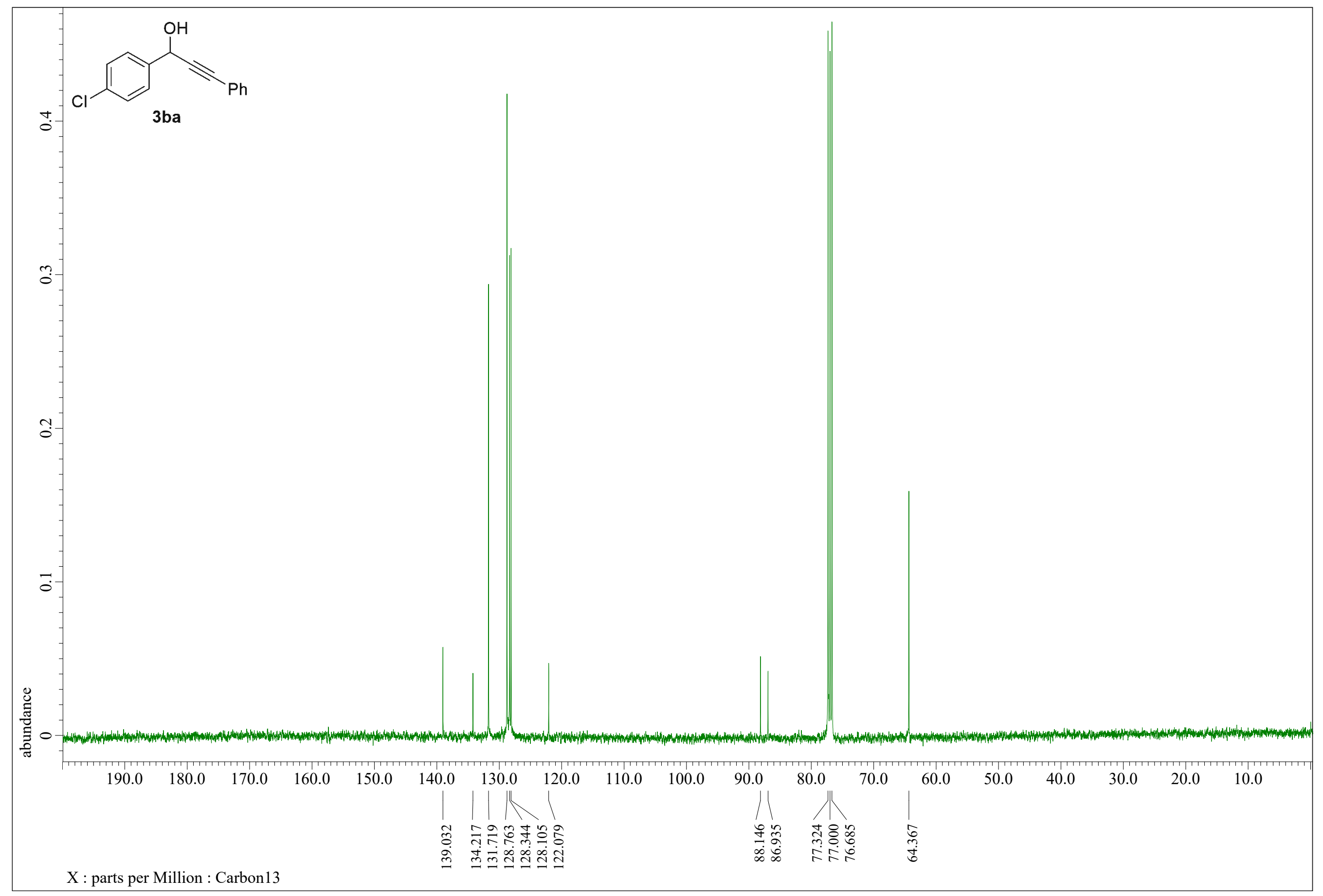


$400 \mathrm{MHz}, \mathrm{CDCl}_{3}$

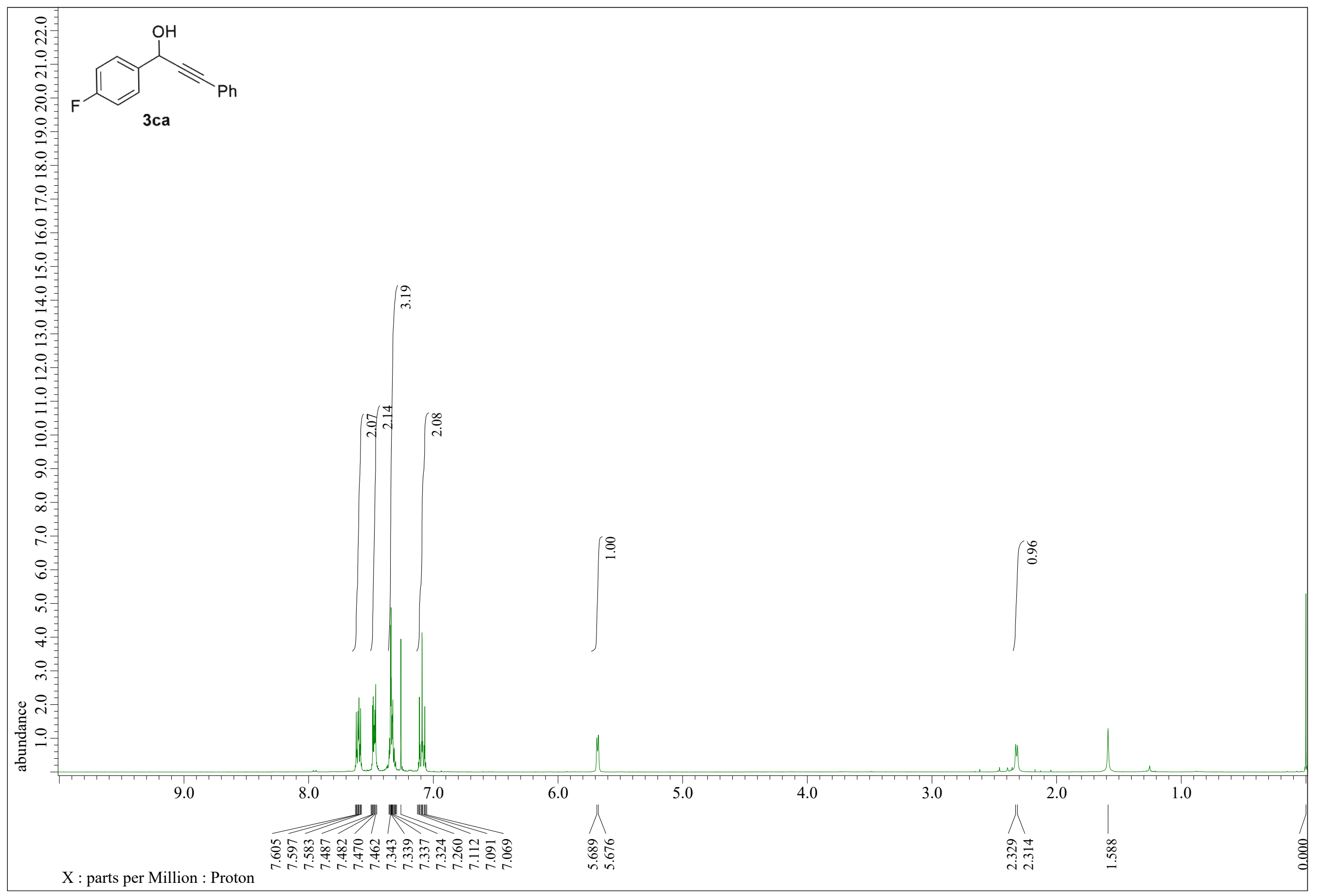


$100.5 \mathrm{MHz}, \mathrm{CDCl}_{3}$

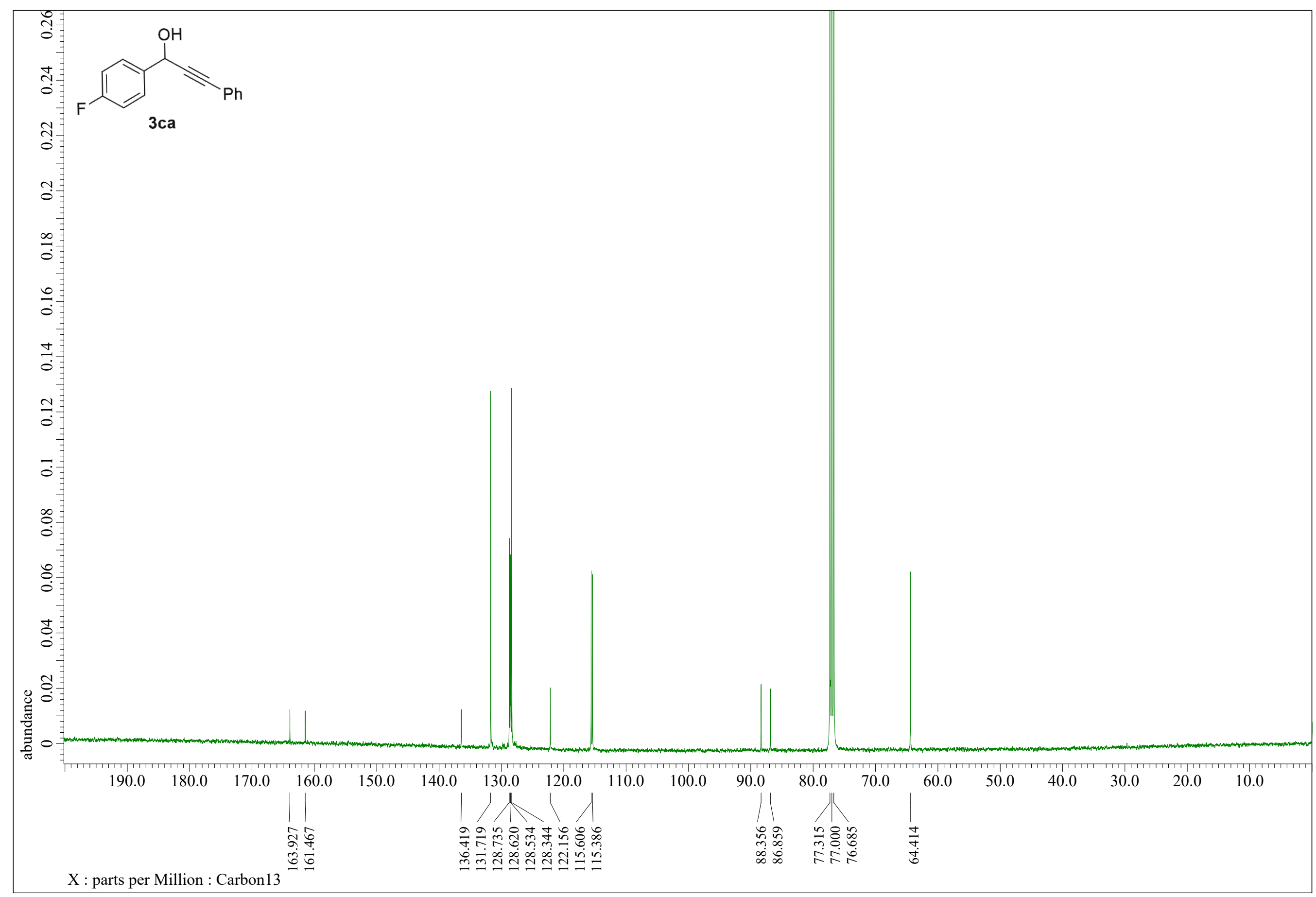


$400 \mathrm{MHz}, \mathrm{CDCl}_{3}$

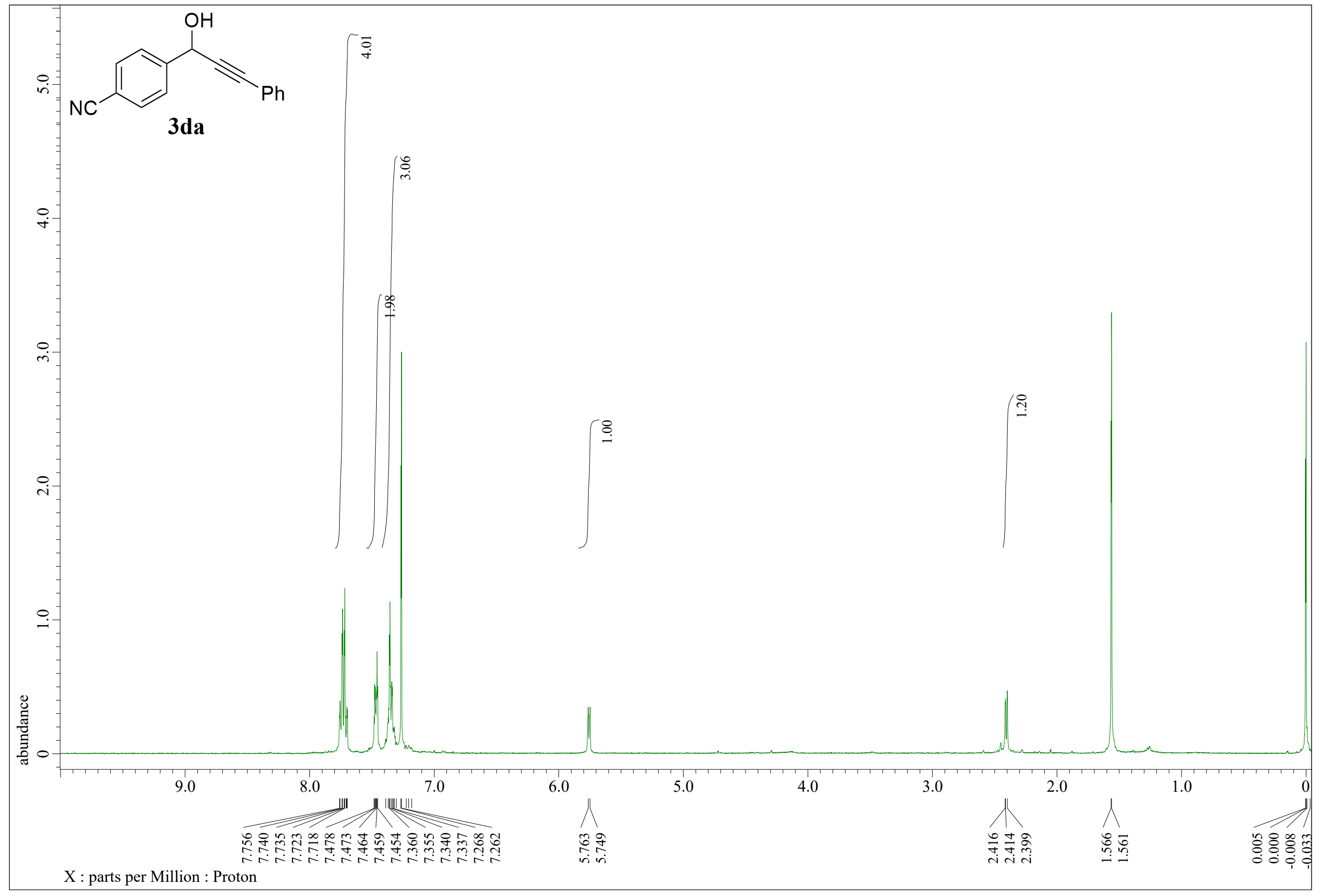


$100.5 \mathrm{MHz}, \mathrm{CDCl}_{3}$

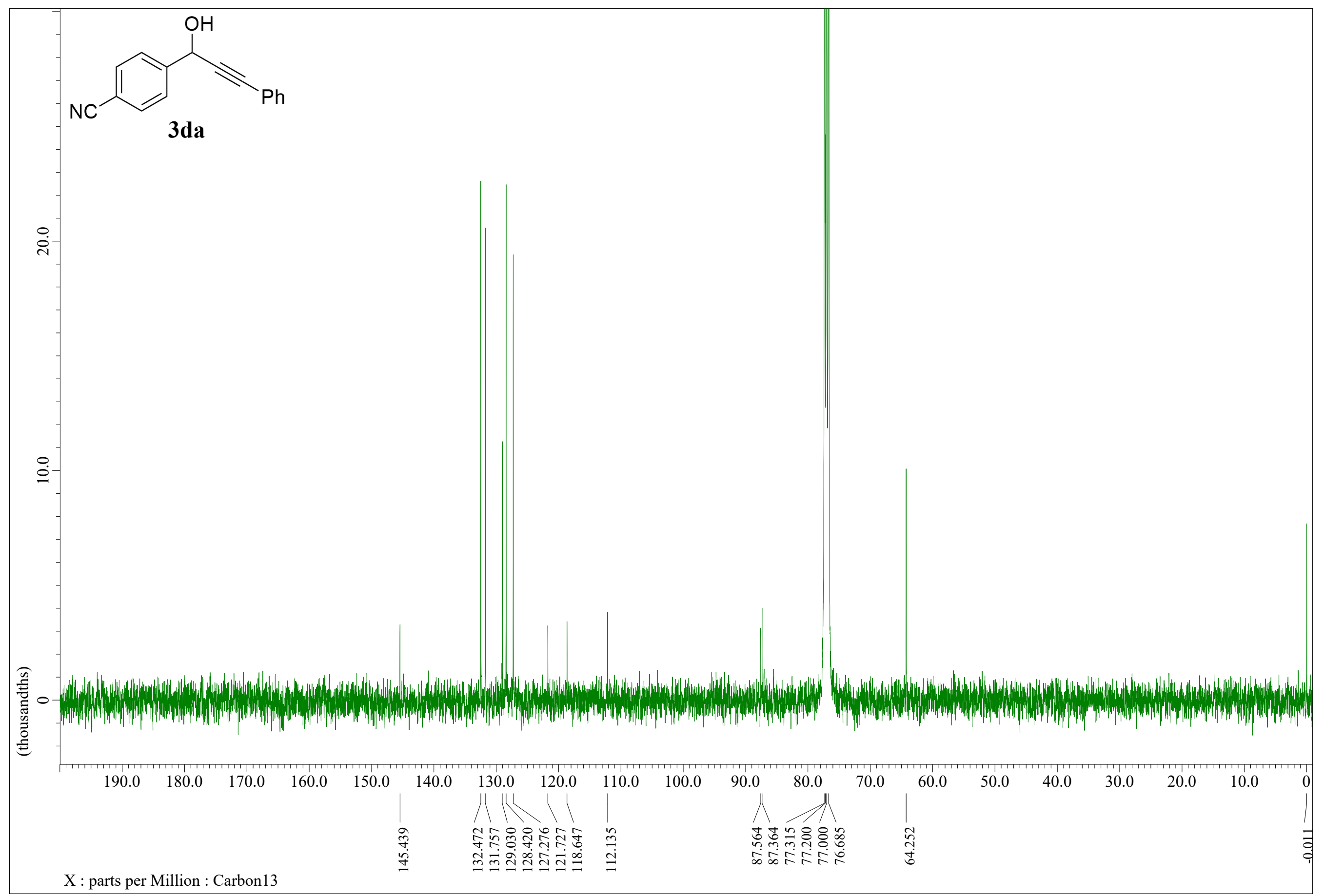


$400 \mathrm{MHz}, \mathrm{CDCl}_{3}$

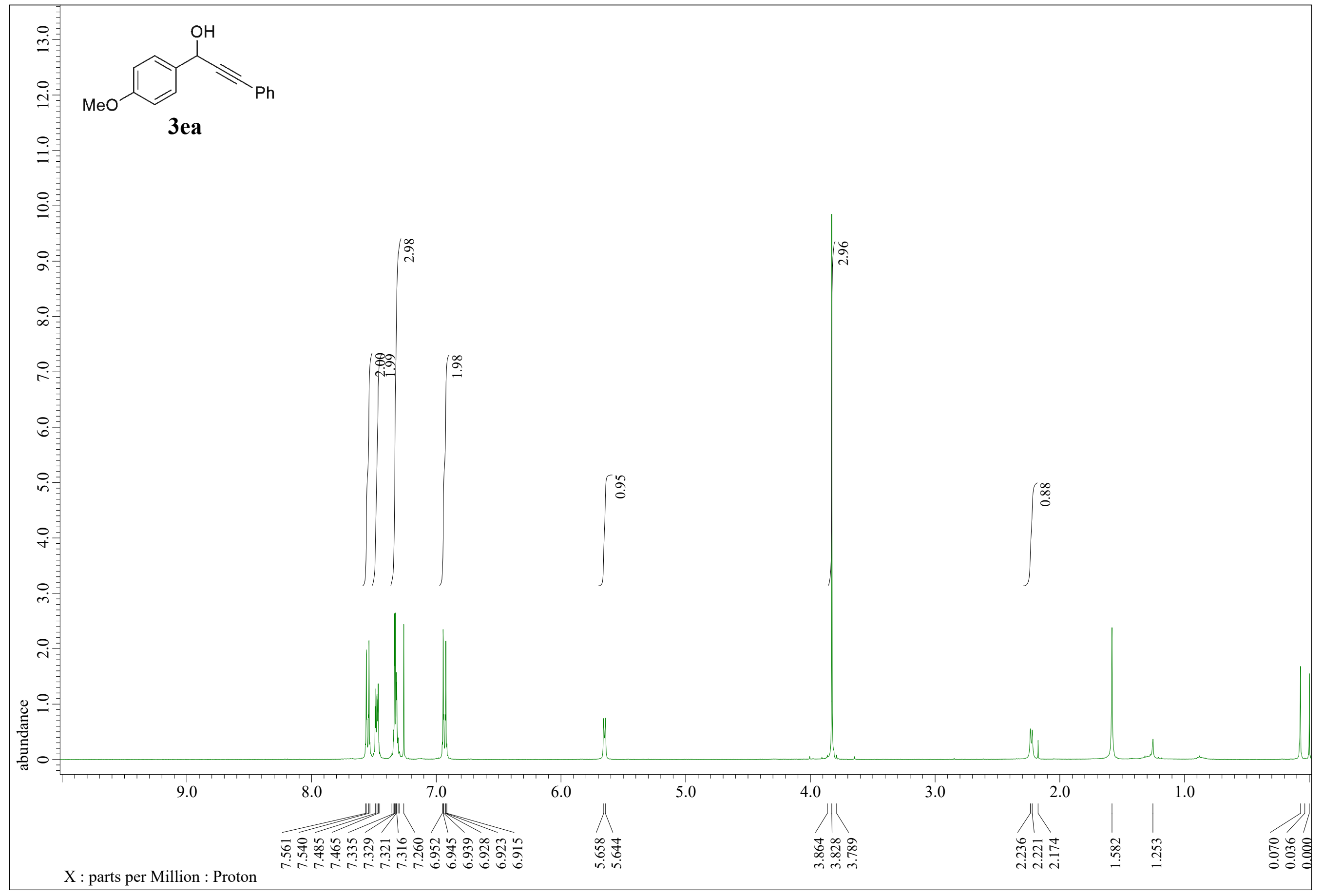


$100.5 \mathrm{MHz}, \mathrm{CDCl}_{3}$

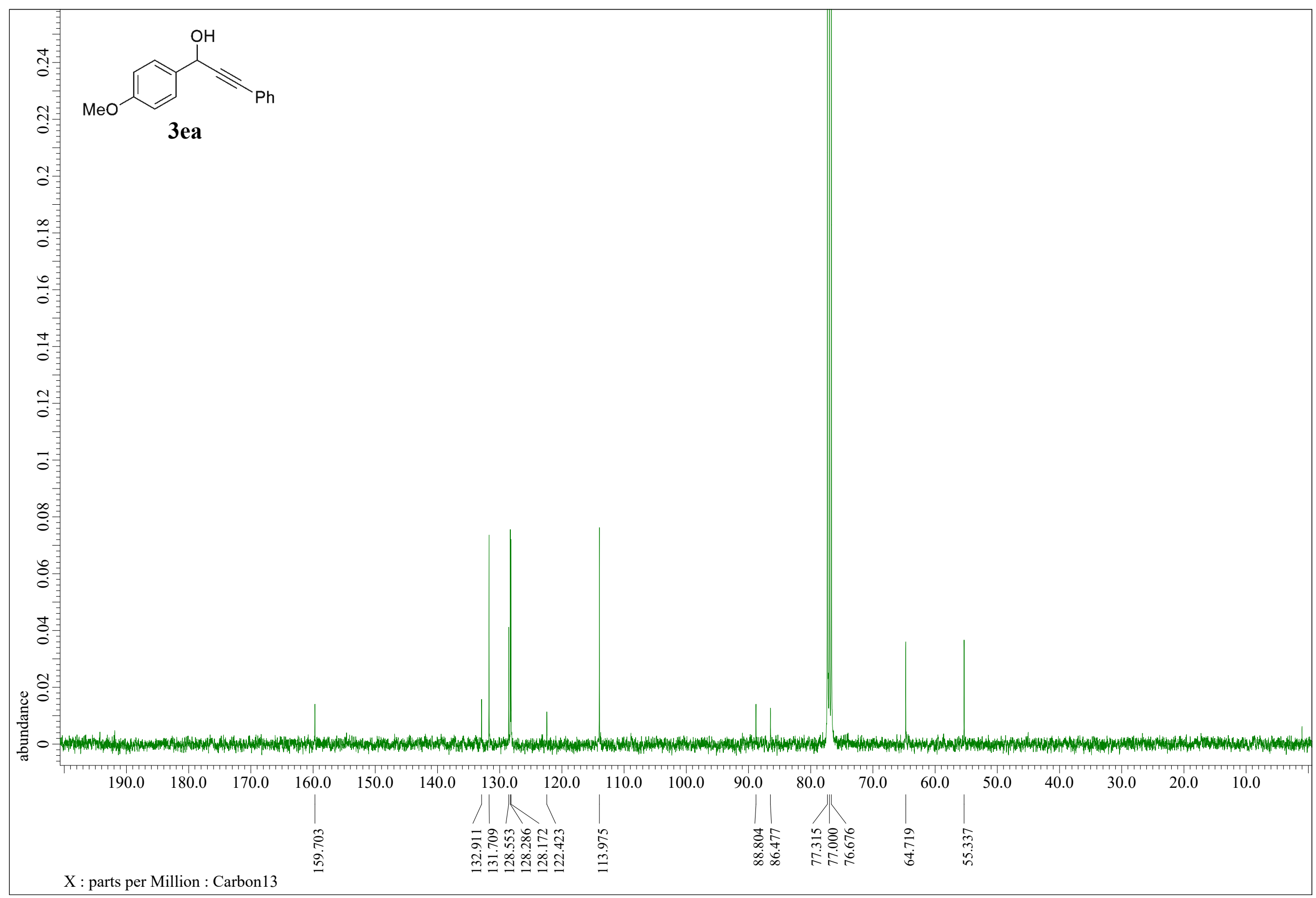


$400 \mathrm{MHz}, \mathrm{CDCl}_{3}$

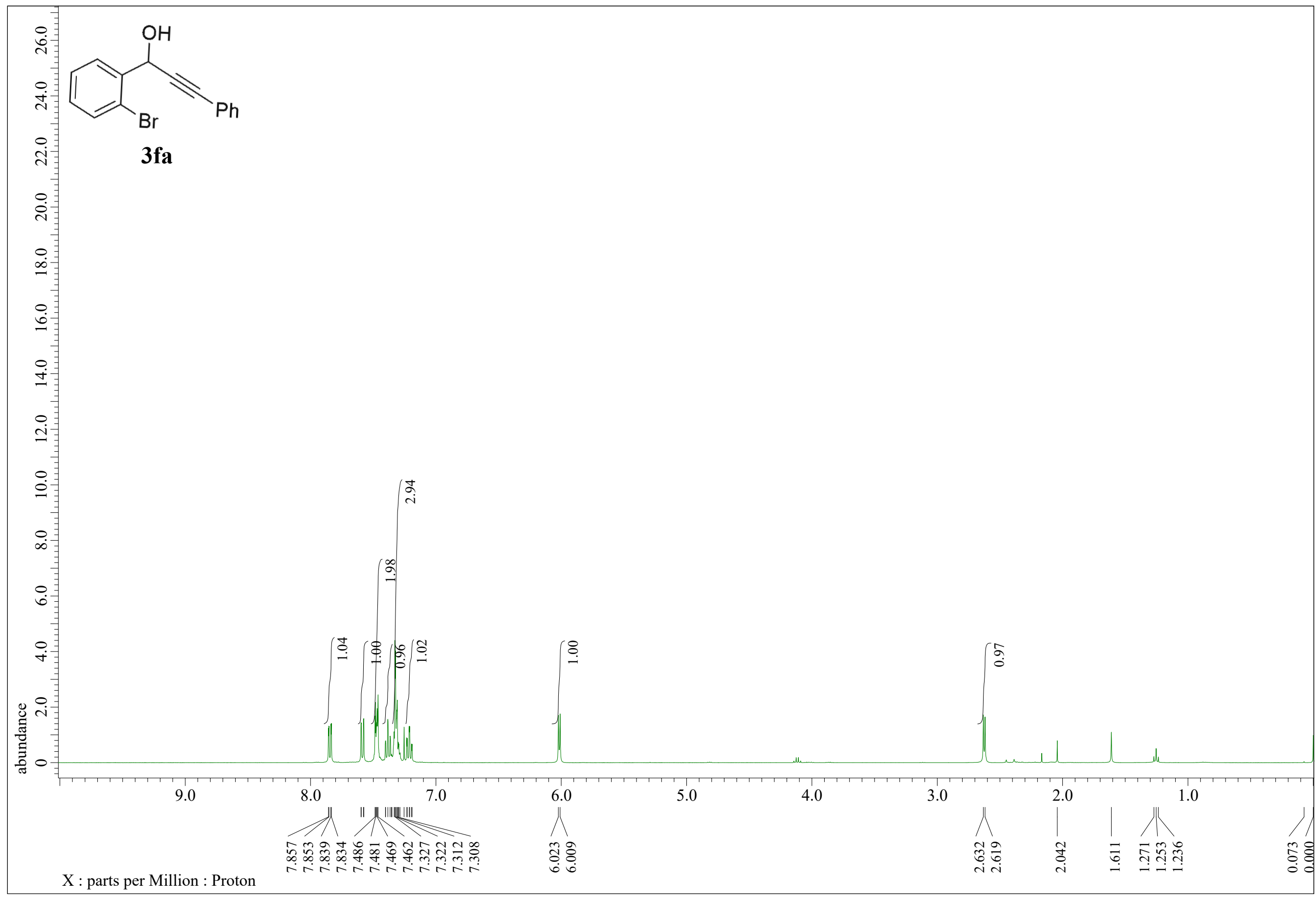


$100.5 \mathrm{MHz}, \mathrm{CDCl}_{3}$

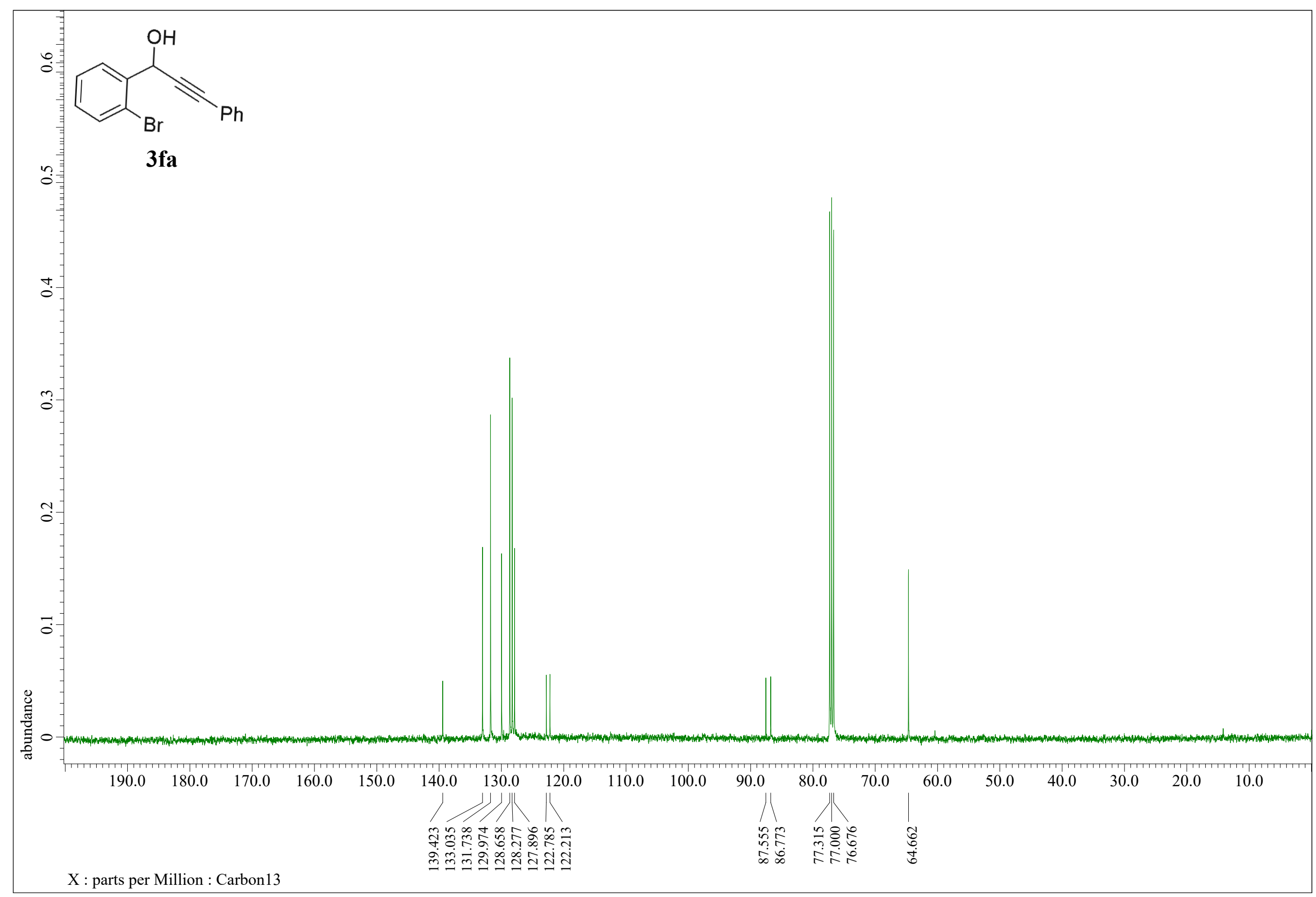


$400 \mathrm{MHz}, \mathrm{CDCl}_{3}$

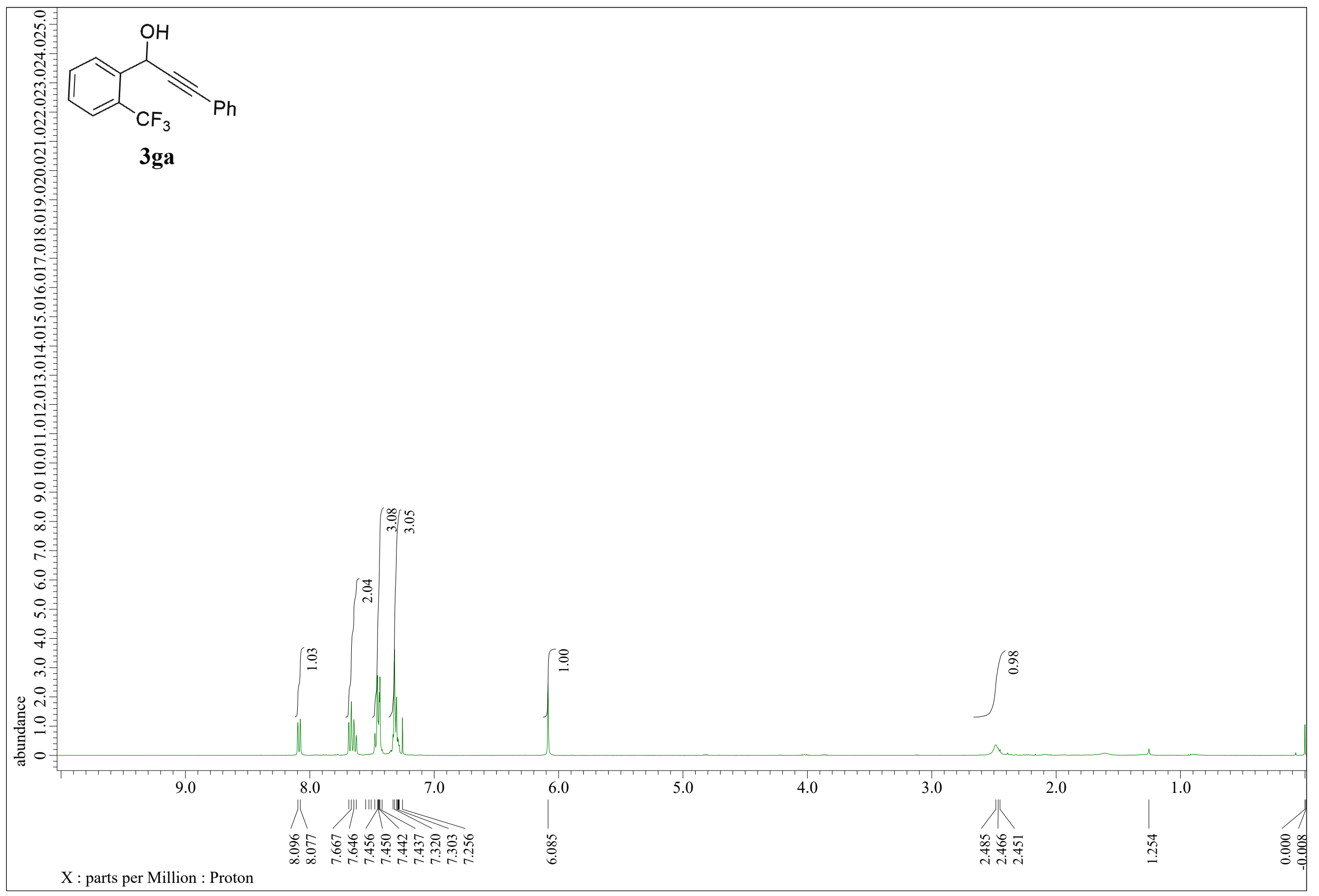


$100.5 \mathrm{MHz}, \mathrm{CDCl}_{3}$

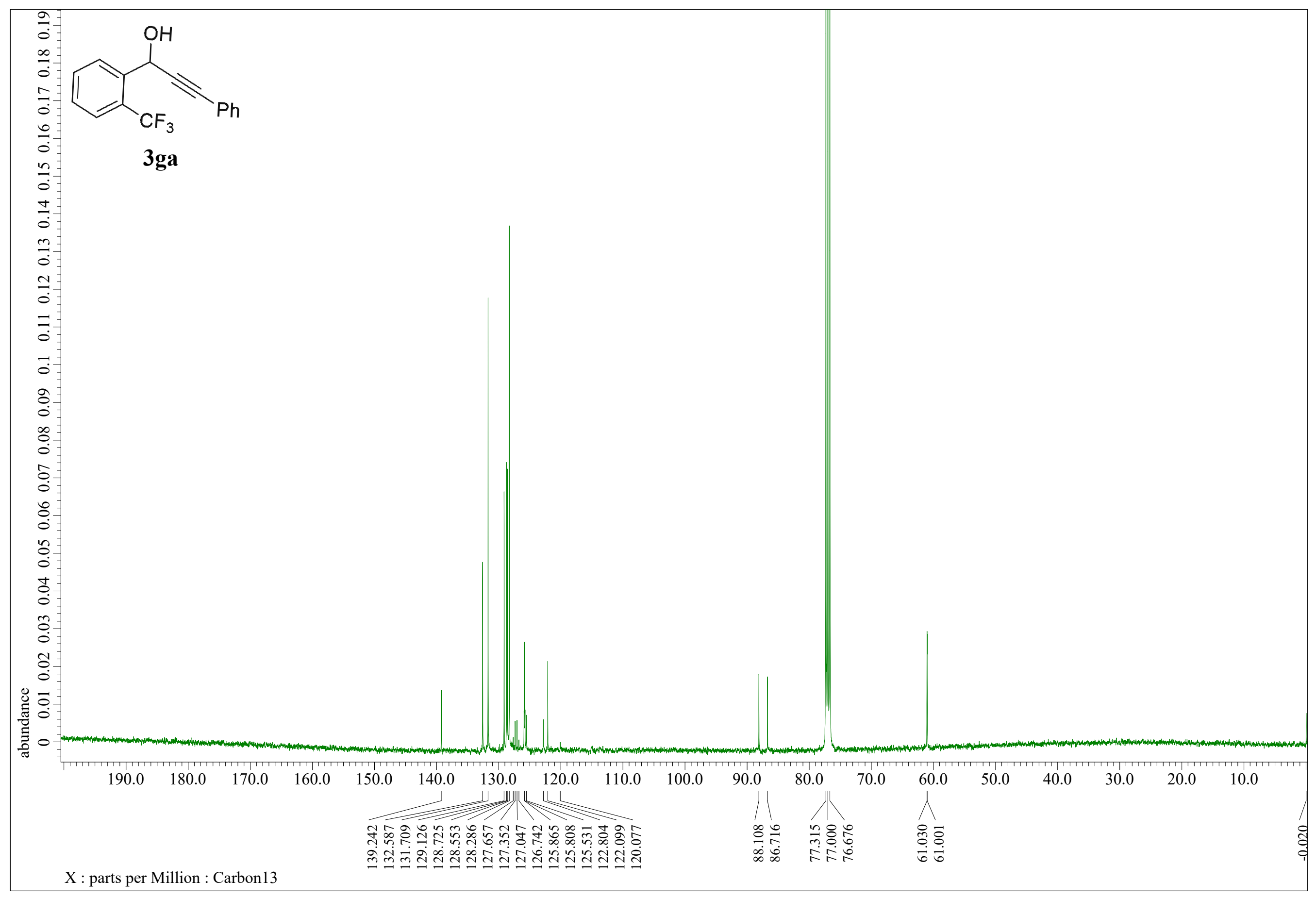


$400 \mathrm{MHz}, \mathrm{CDCl}_{3}$

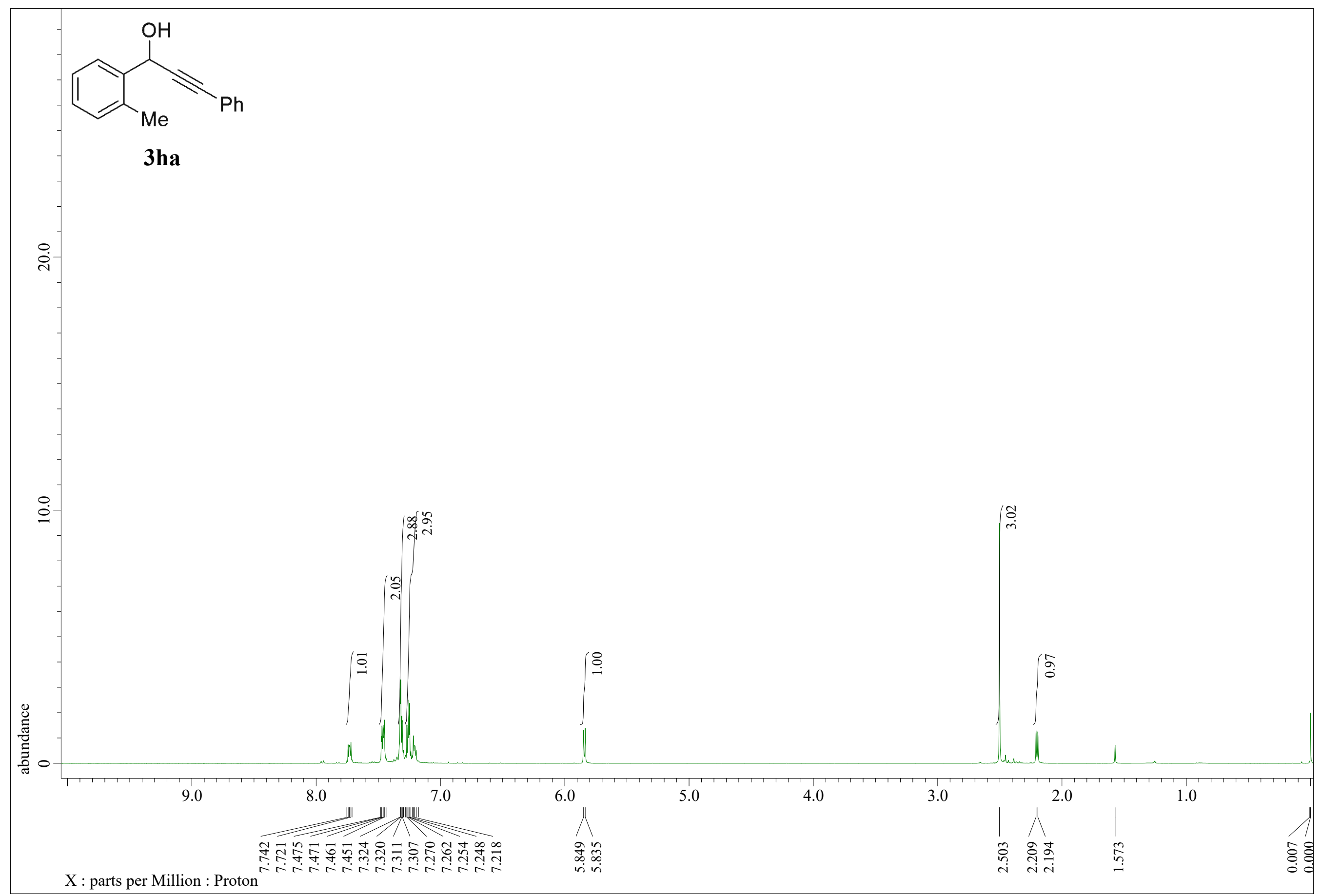


$100.5 \mathrm{MHz}, \mathrm{CDCl}_{3}$

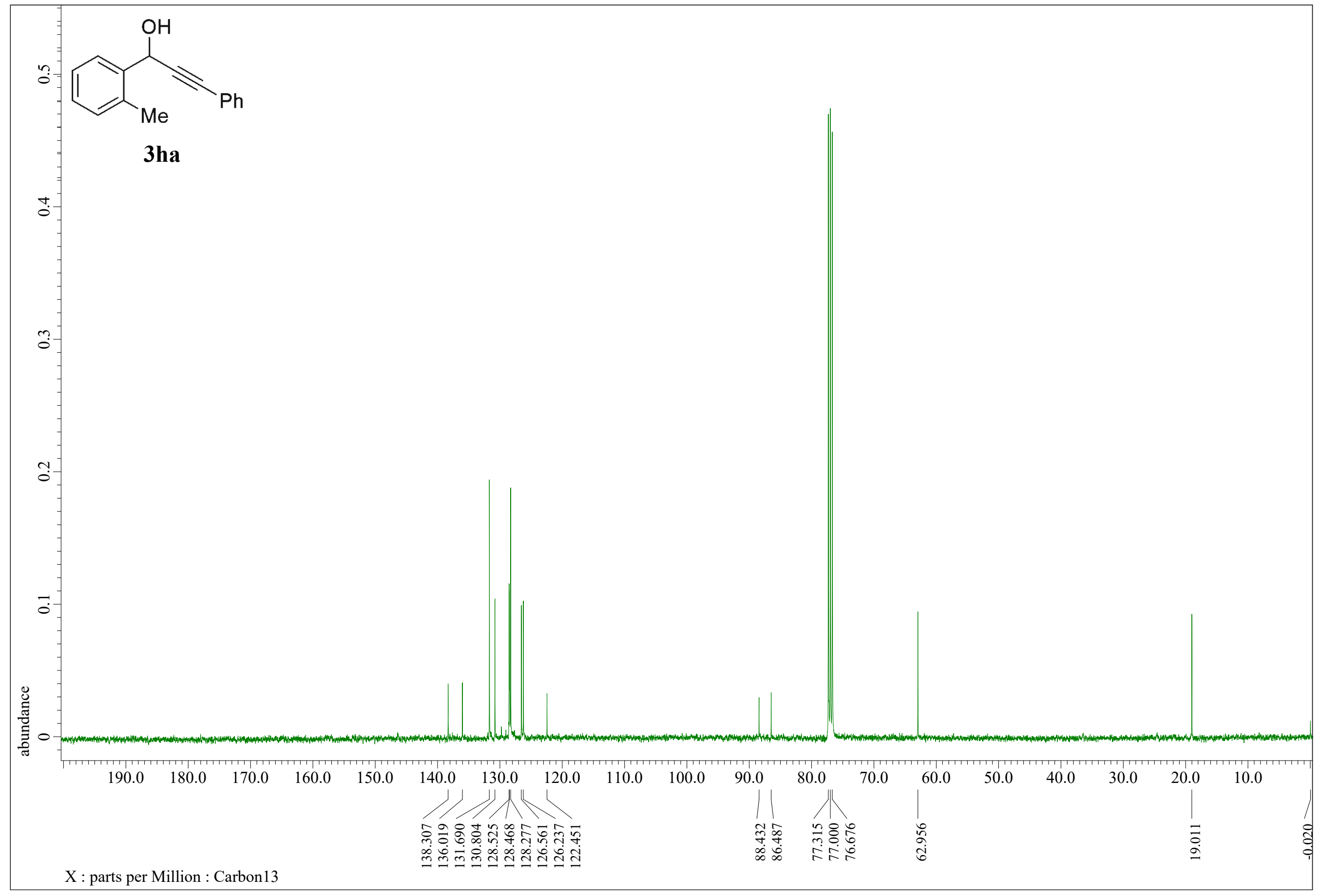


$400 \mathrm{MHz}, \mathrm{CDCl}_{3}$

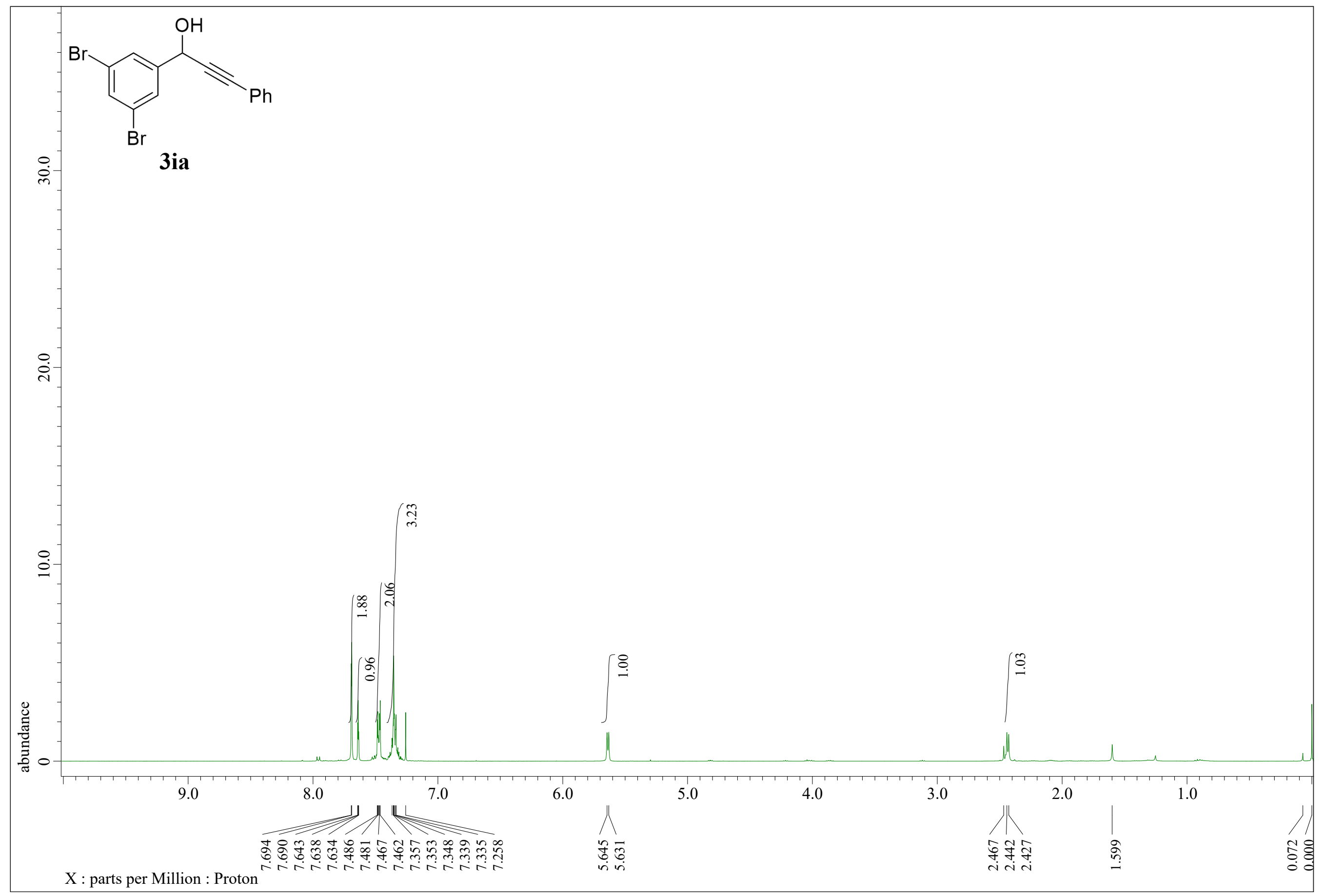


$100.5 \mathrm{MHz}, \mathrm{CDCl}_{3}$

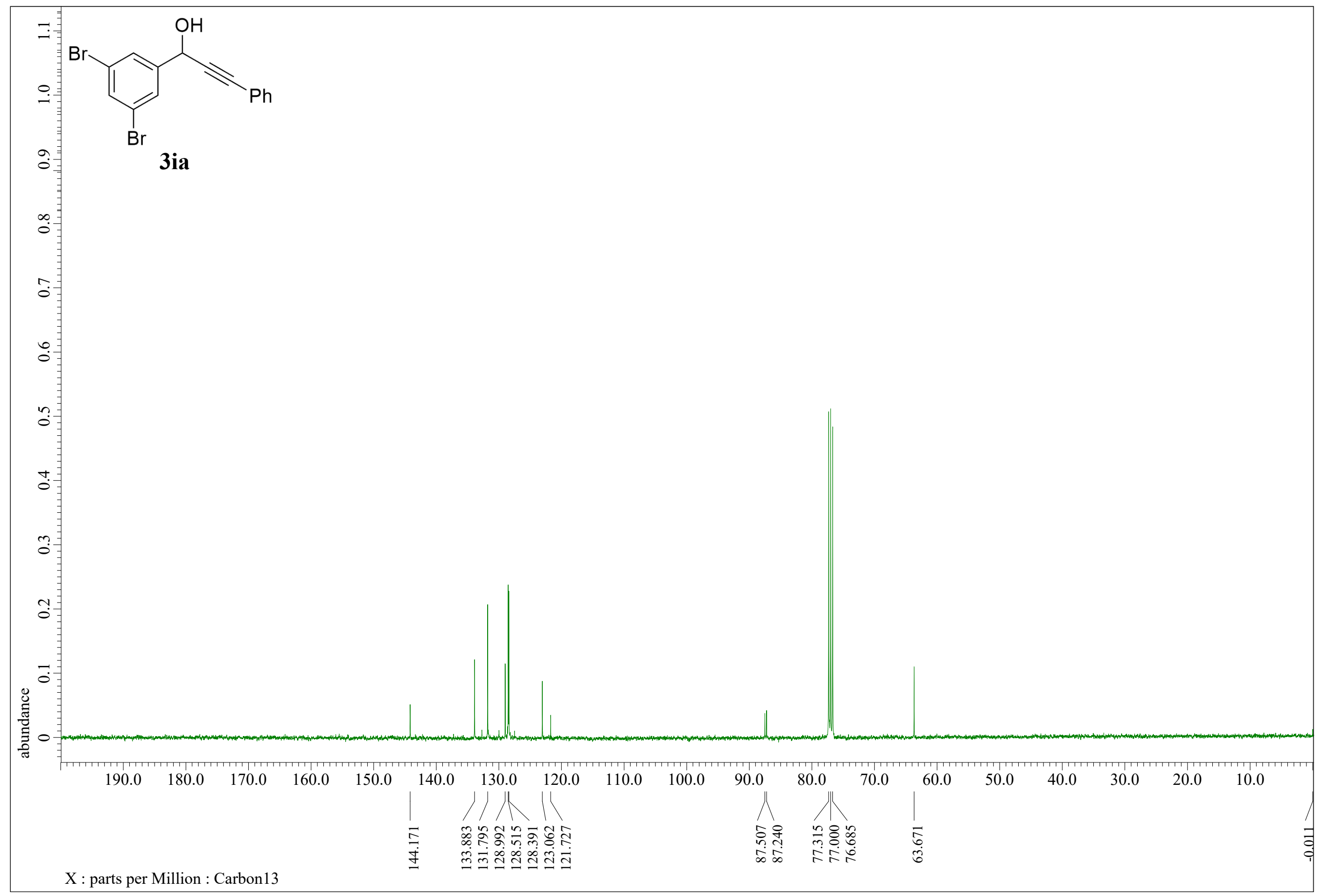


$400 \mathrm{MHz}, \mathrm{CDCl}_{3}$

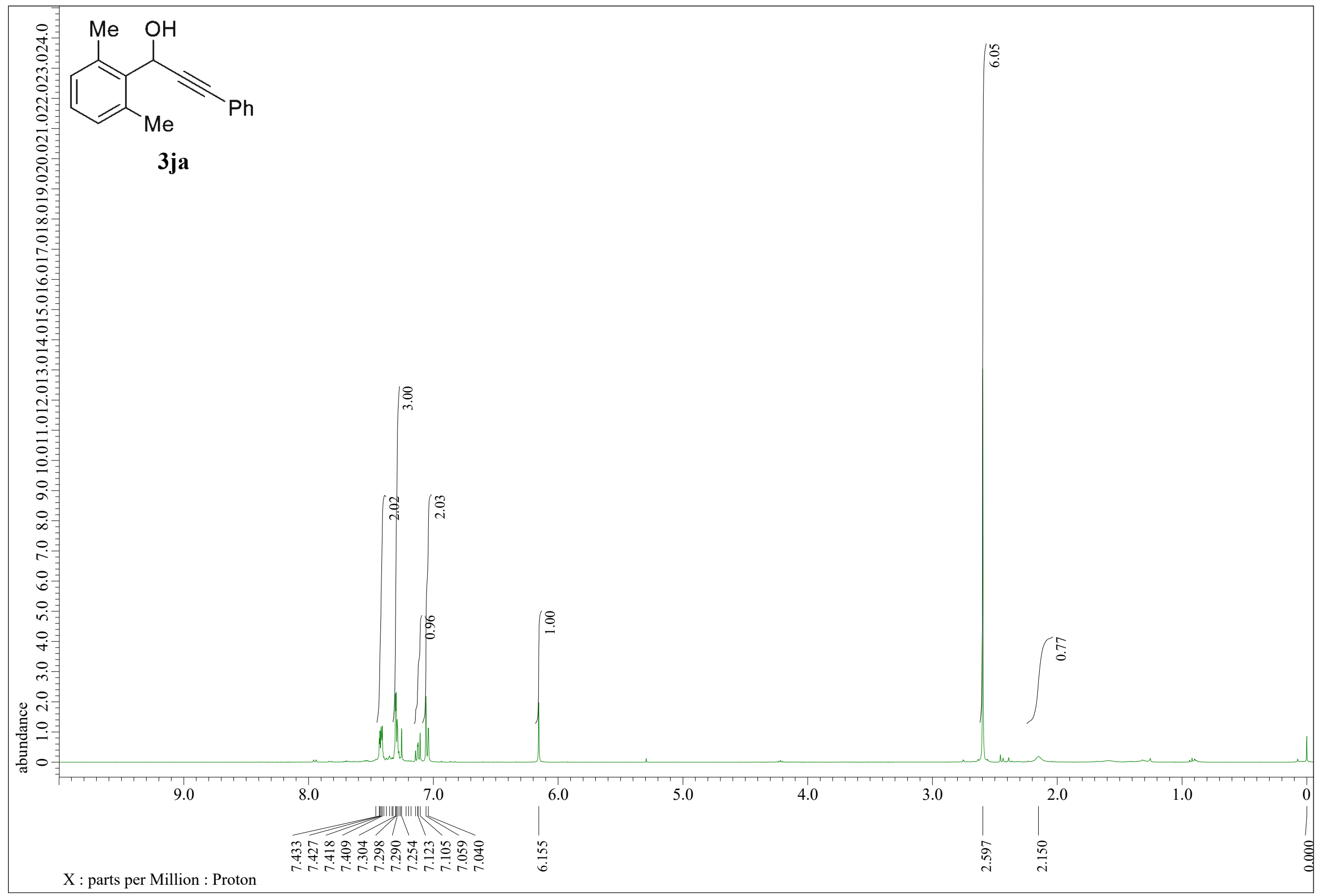


$100.5 \mathrm{MHz}, \mathrm{CDCl}_{3}$

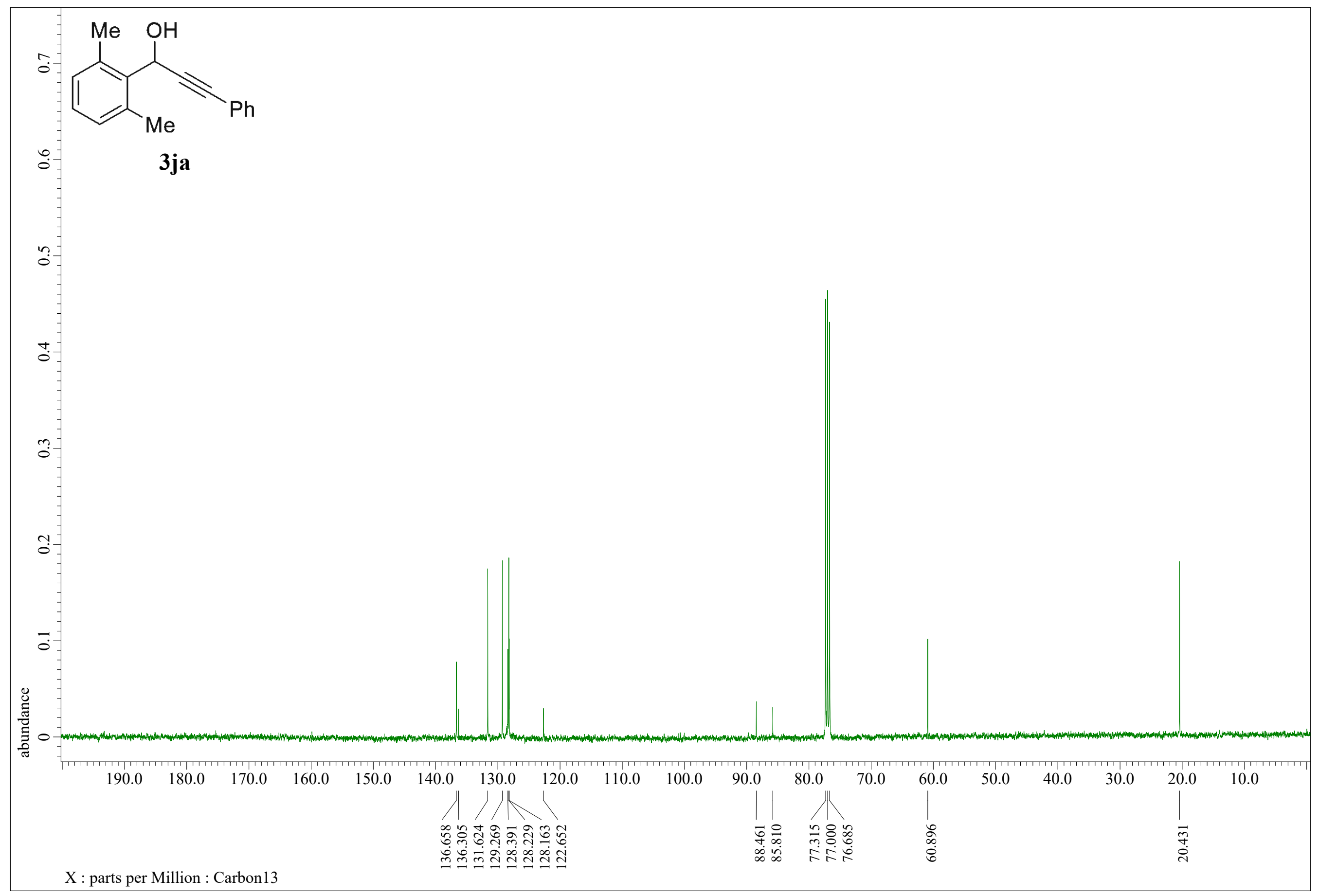


$400 \mathrm{MHz}, \mathrm{CDCl}_{3}$

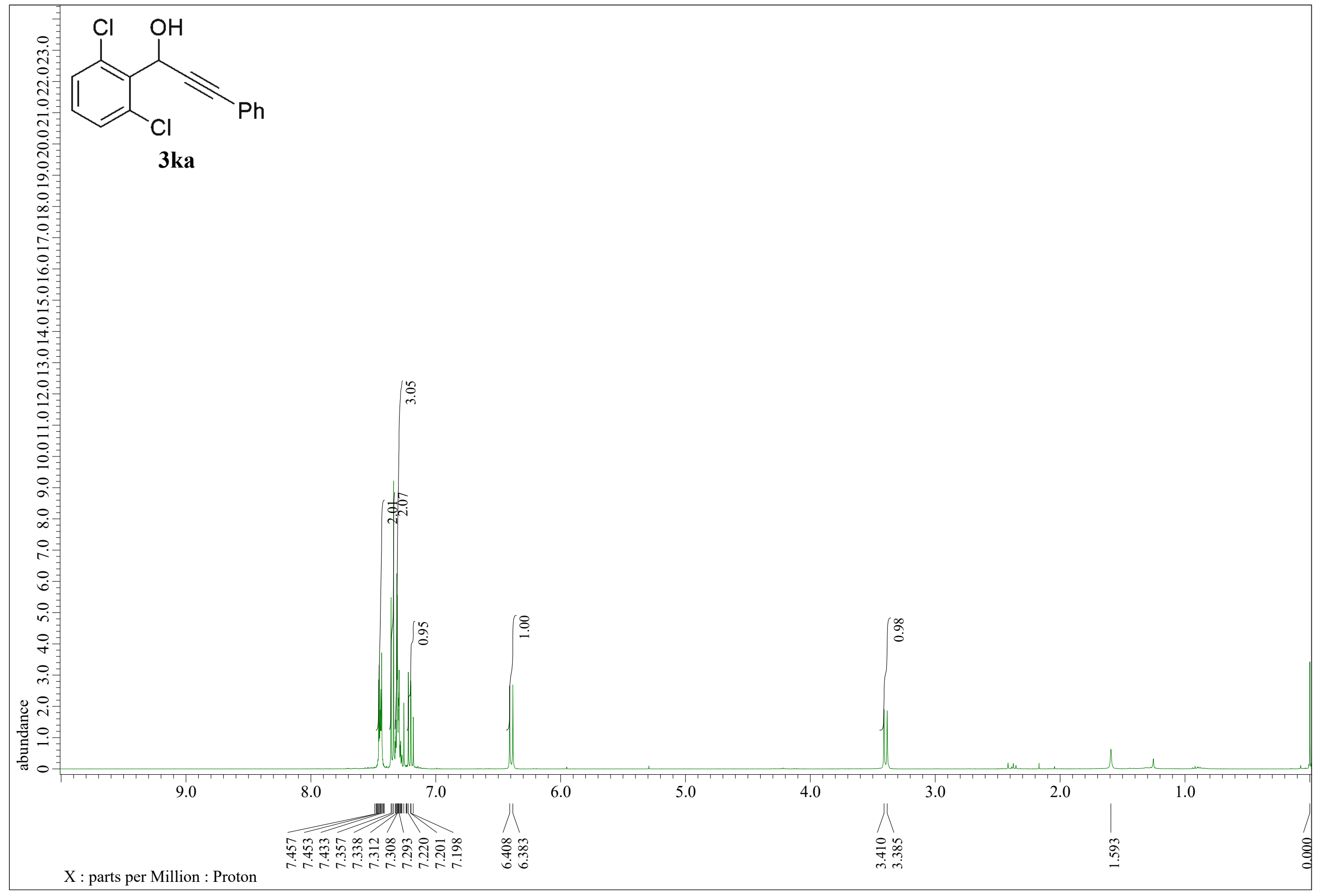


$100.5 \mathrm{MHz}, \mathrm{CDCl}_{3}$

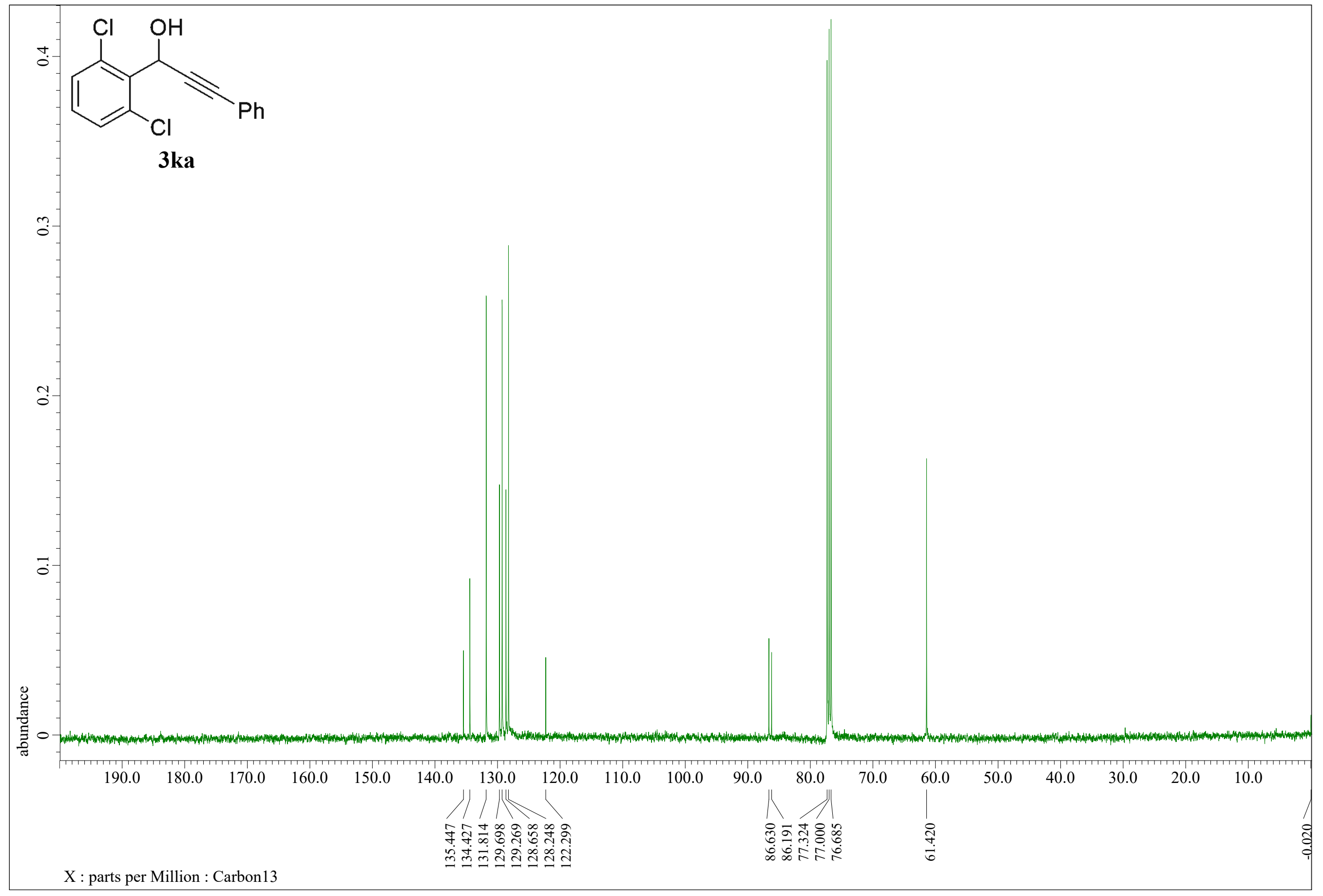


$400 \mathrm{MHz}, \mathrm{CDCl}_{3}$

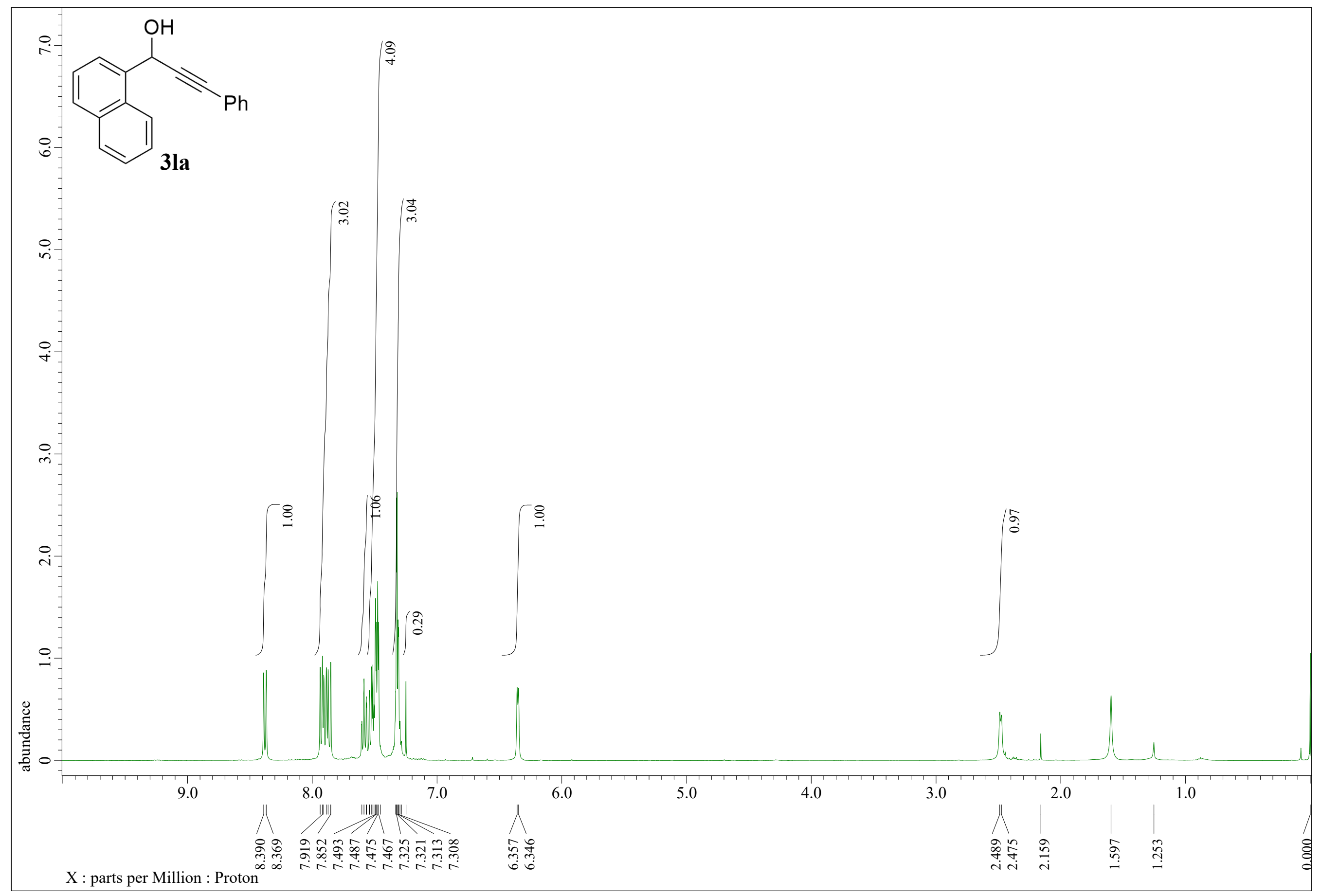


$100.5 \mathrm{MHz}, \mathrm{CDCl}_{3}$

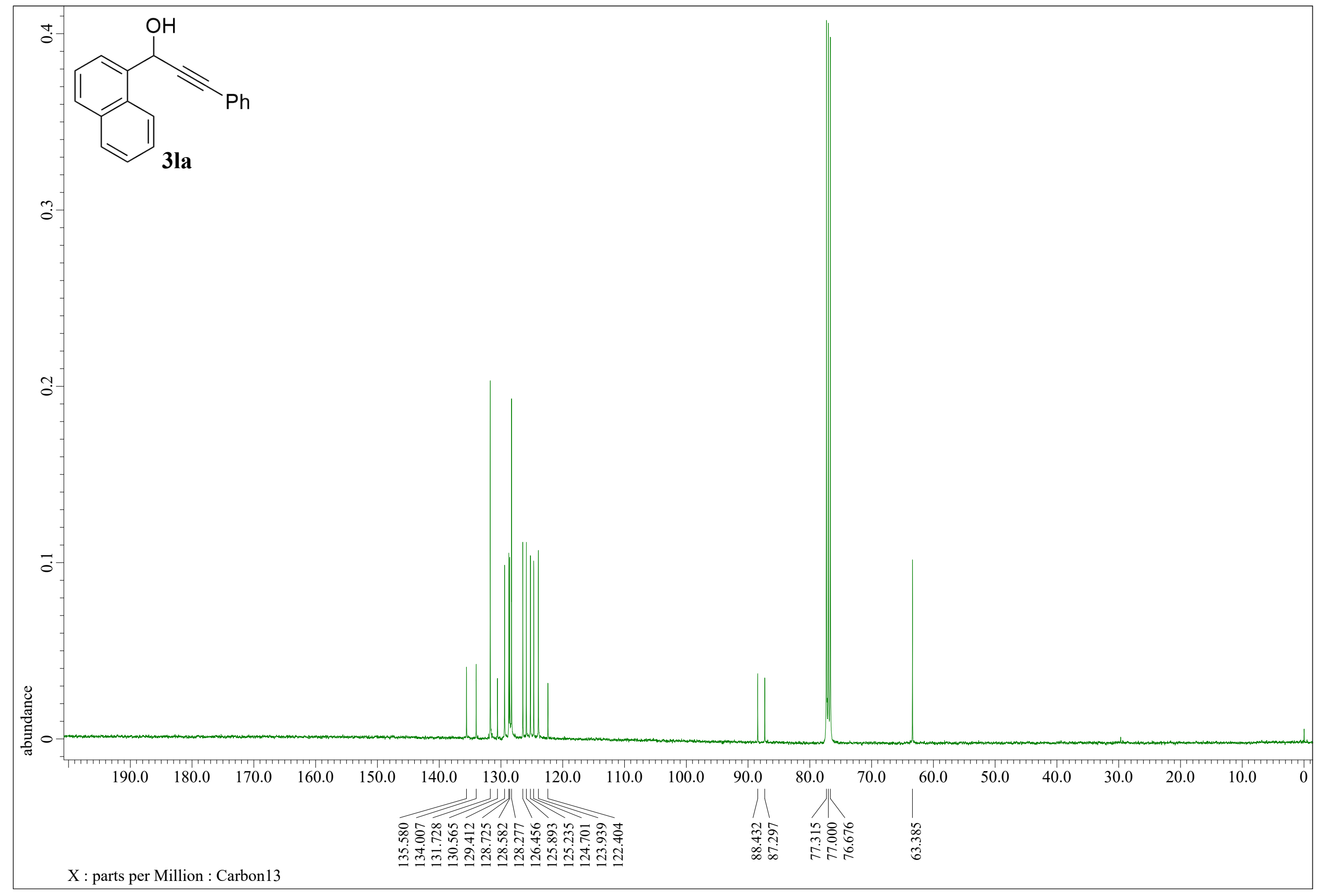


$400 \mathrm{MHz}, \mathrm{CDCl}_{3}$

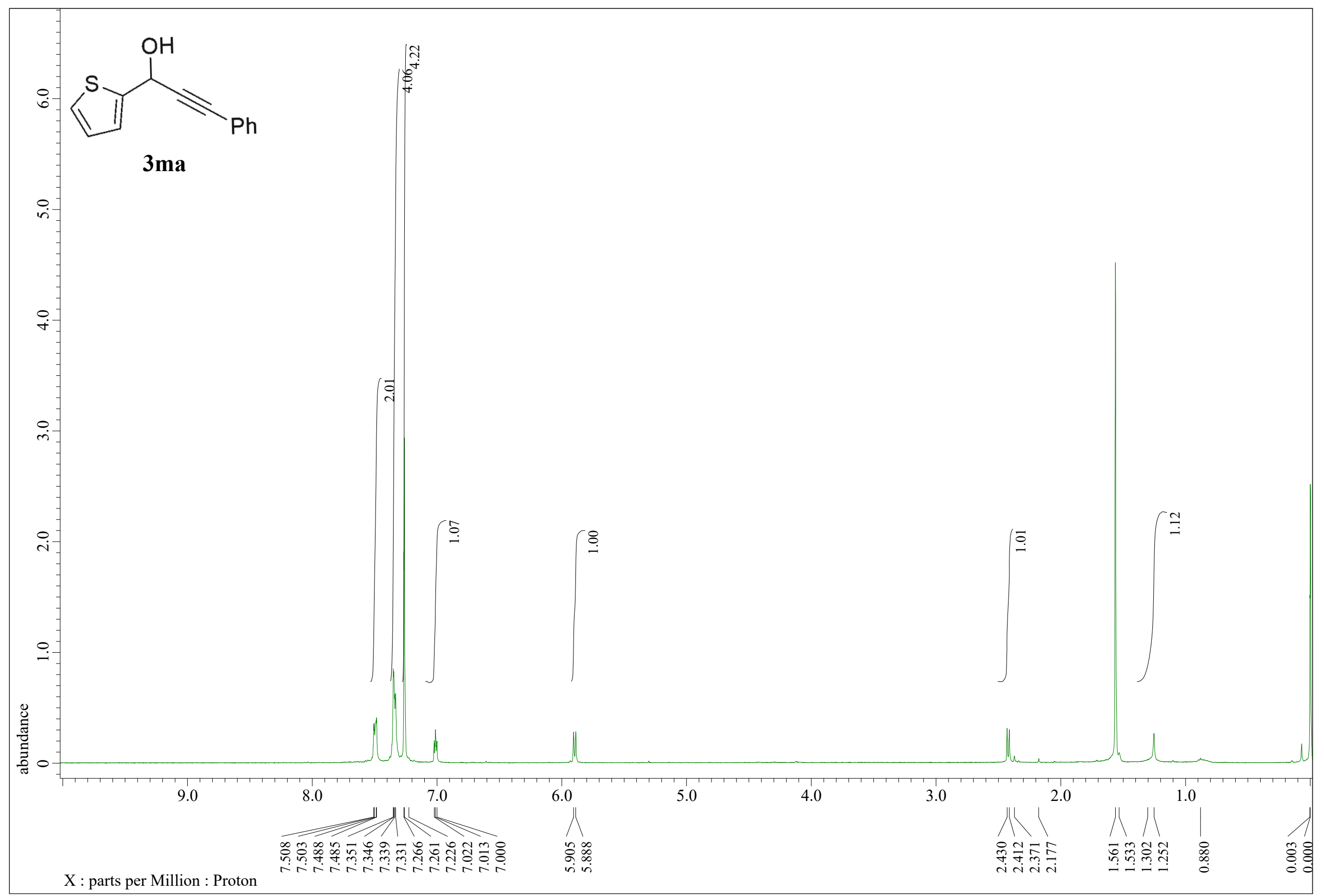


$100.5 \mathrm{MHz}, \mathrm{CDCl}_{3}$

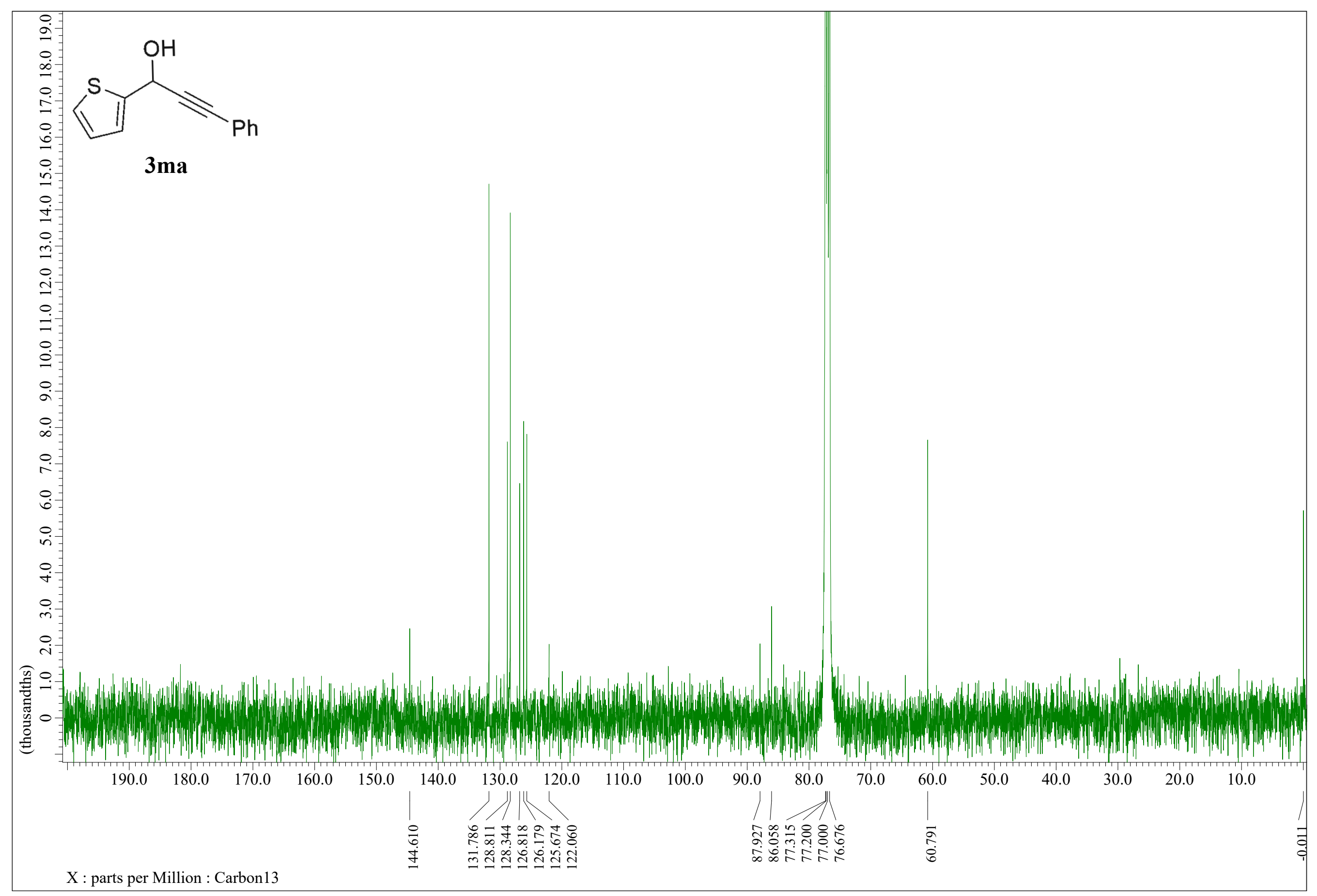


$400 \mathrm{MHz}, \mathrm{CDCl}_{3}$

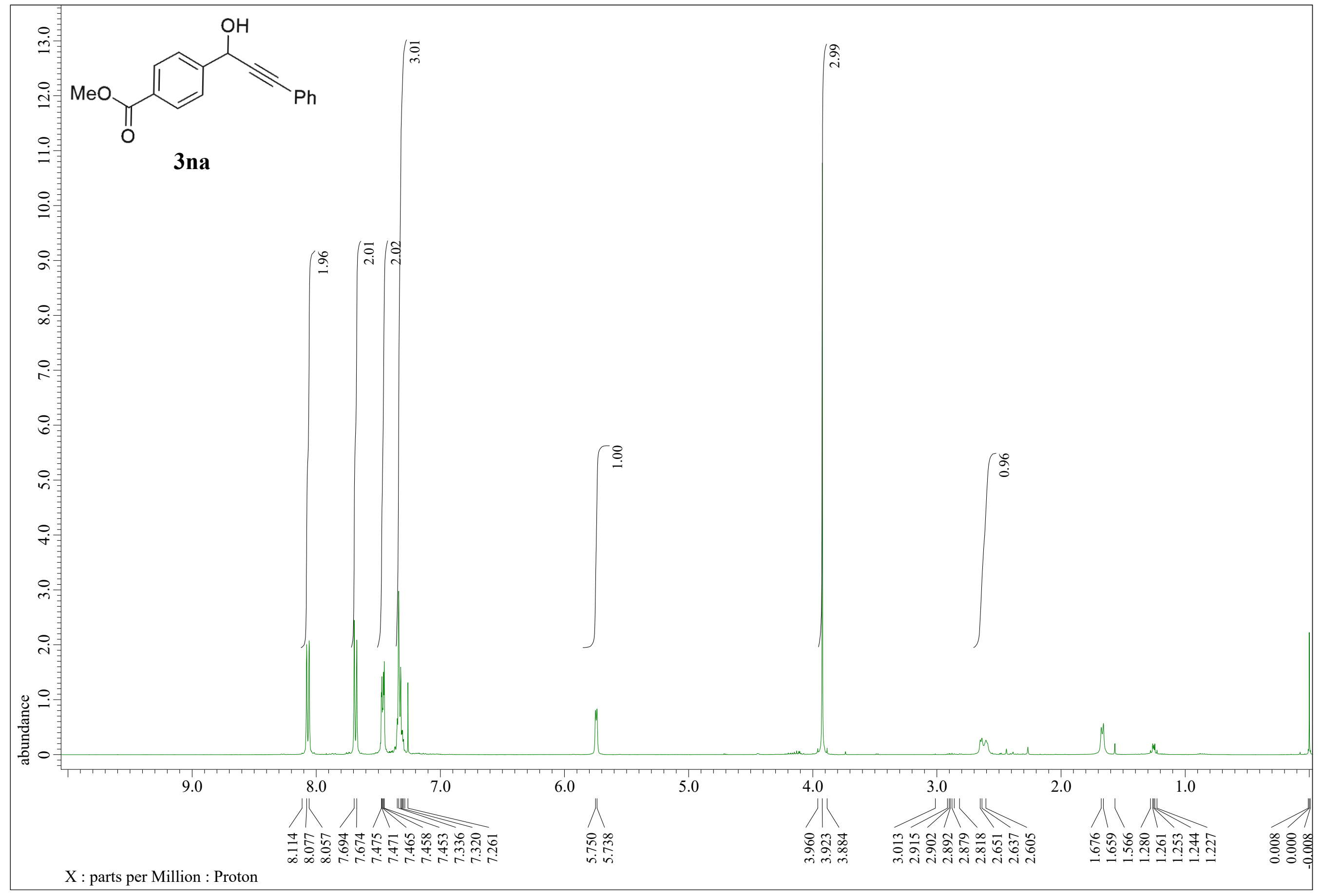




\section{$100.5 \mathrm{MHz}, \mathrm{CDCl}_{3}$}

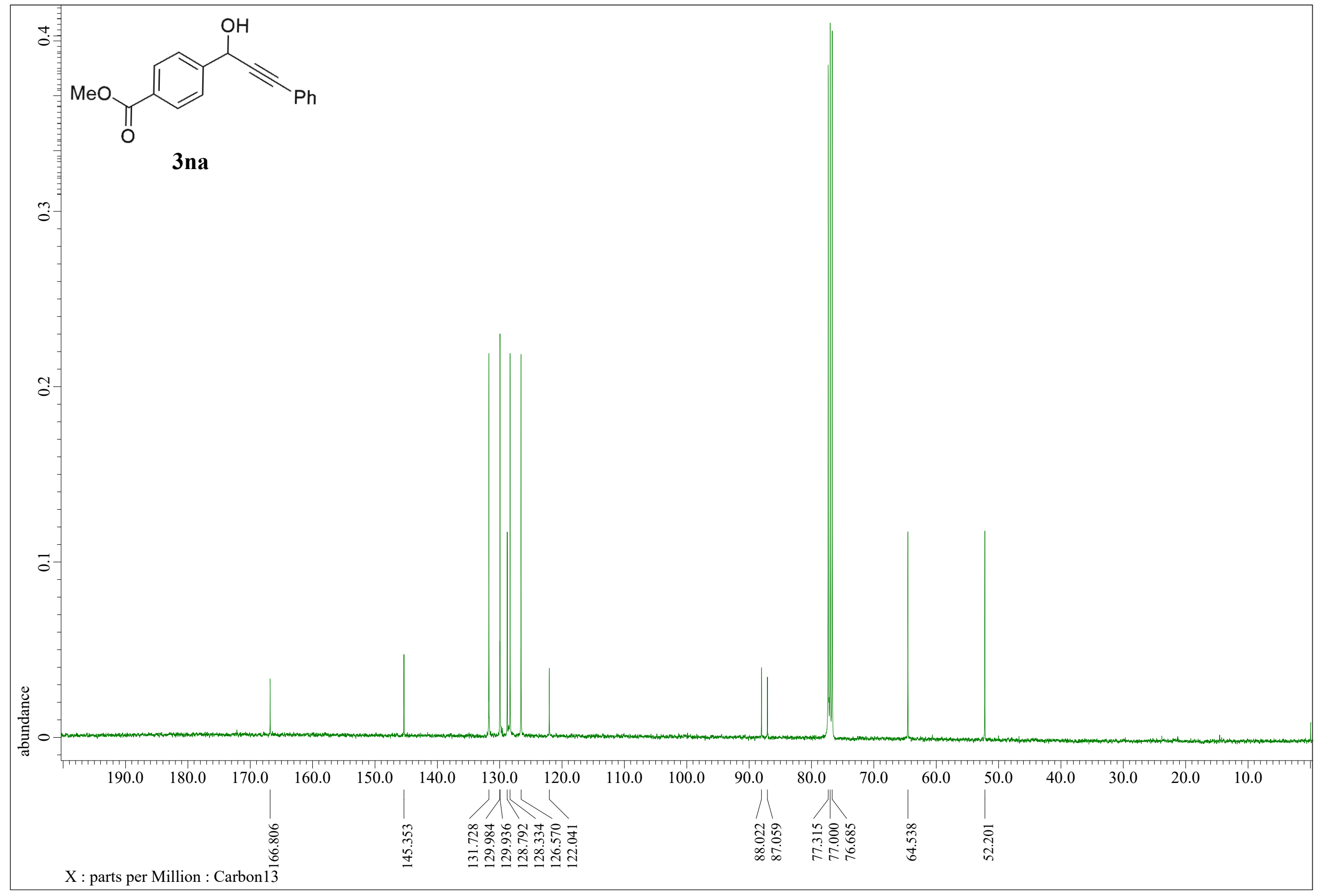


$400 \mathrm{MHz}, \mathrm{CDCl}_{3}$

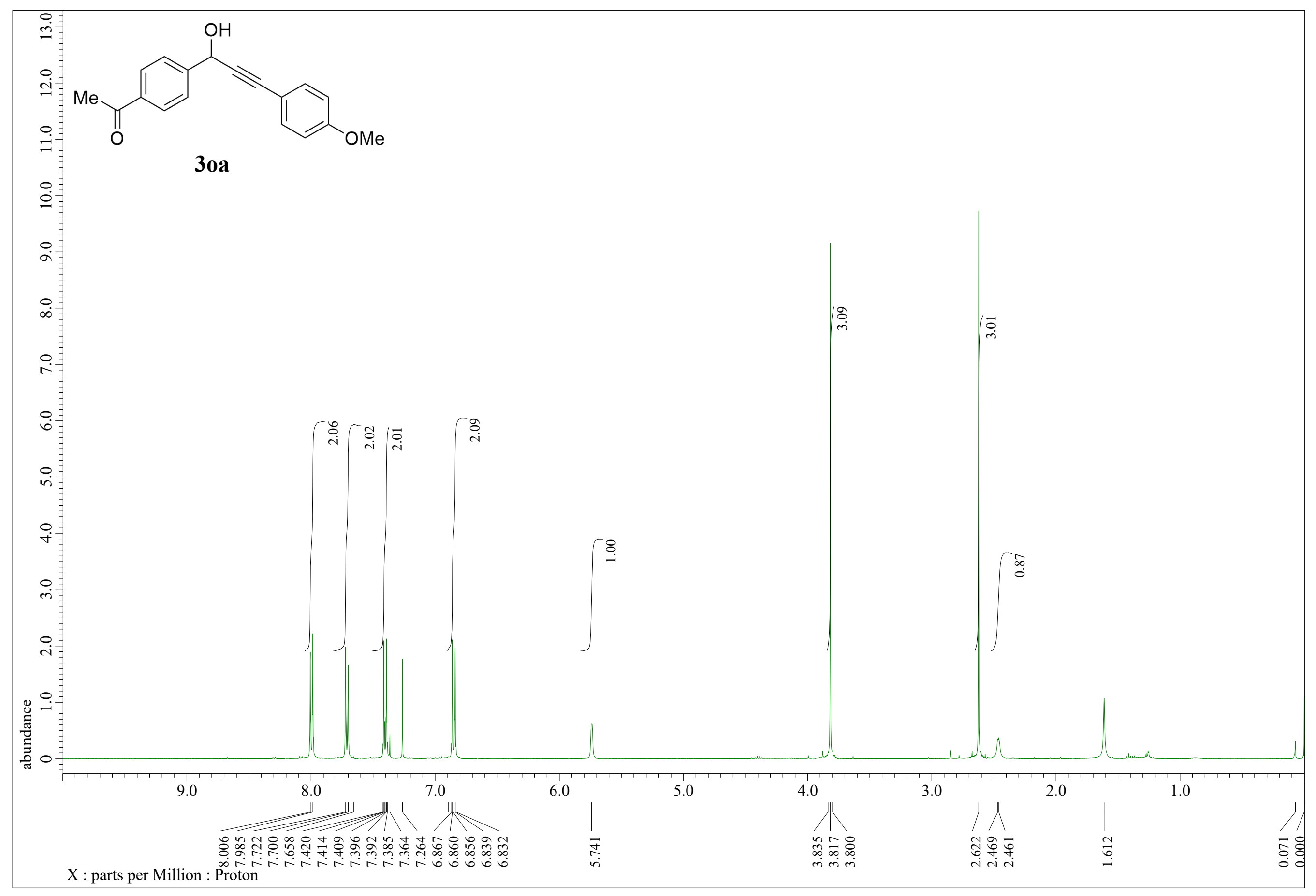


$100.5 \mathrm{MHz}, \mathrm{CDCl}_{3}$

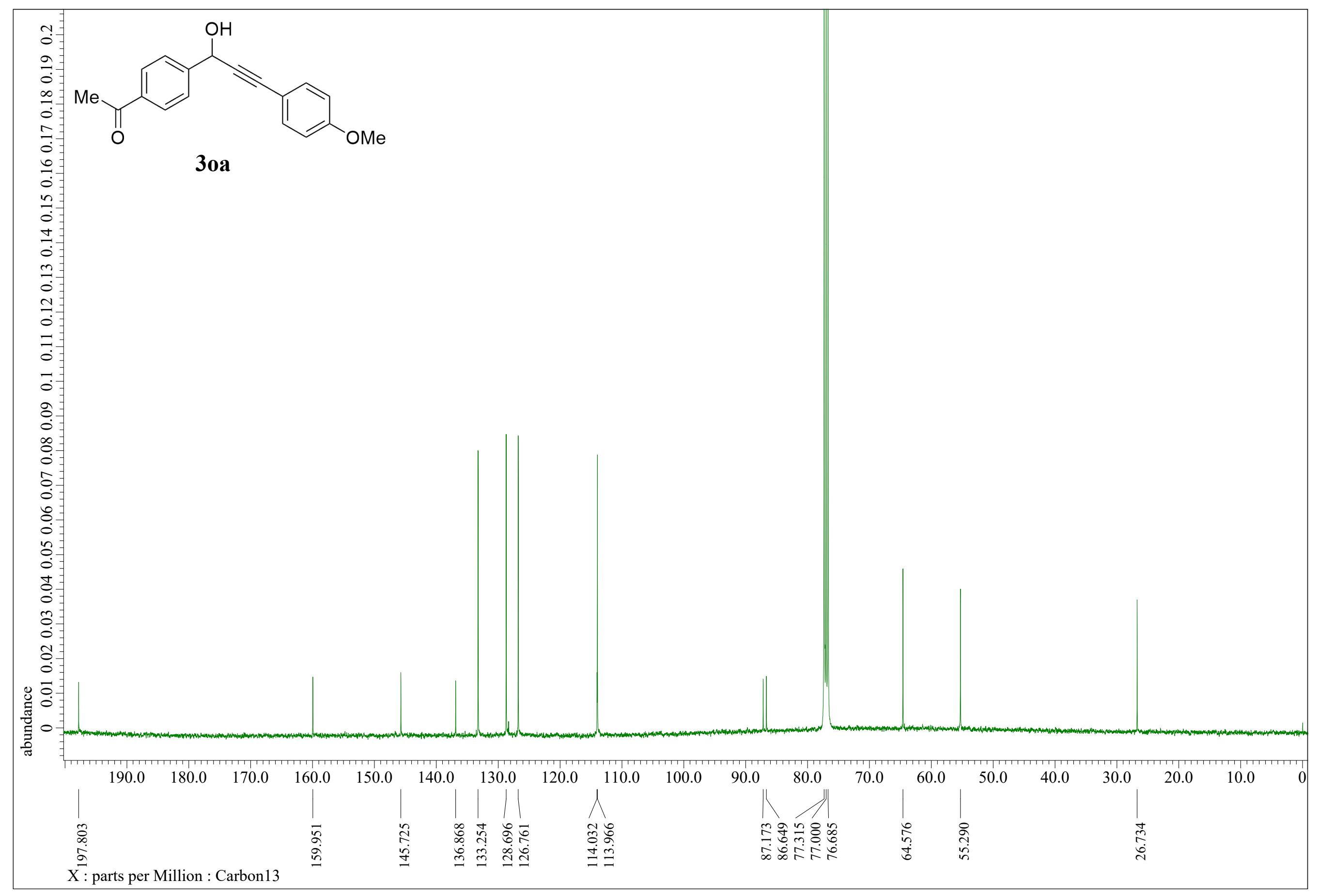


$400 \mathrm{MHz}, \mathrm{CD}_{3} \mathrm{OD}$

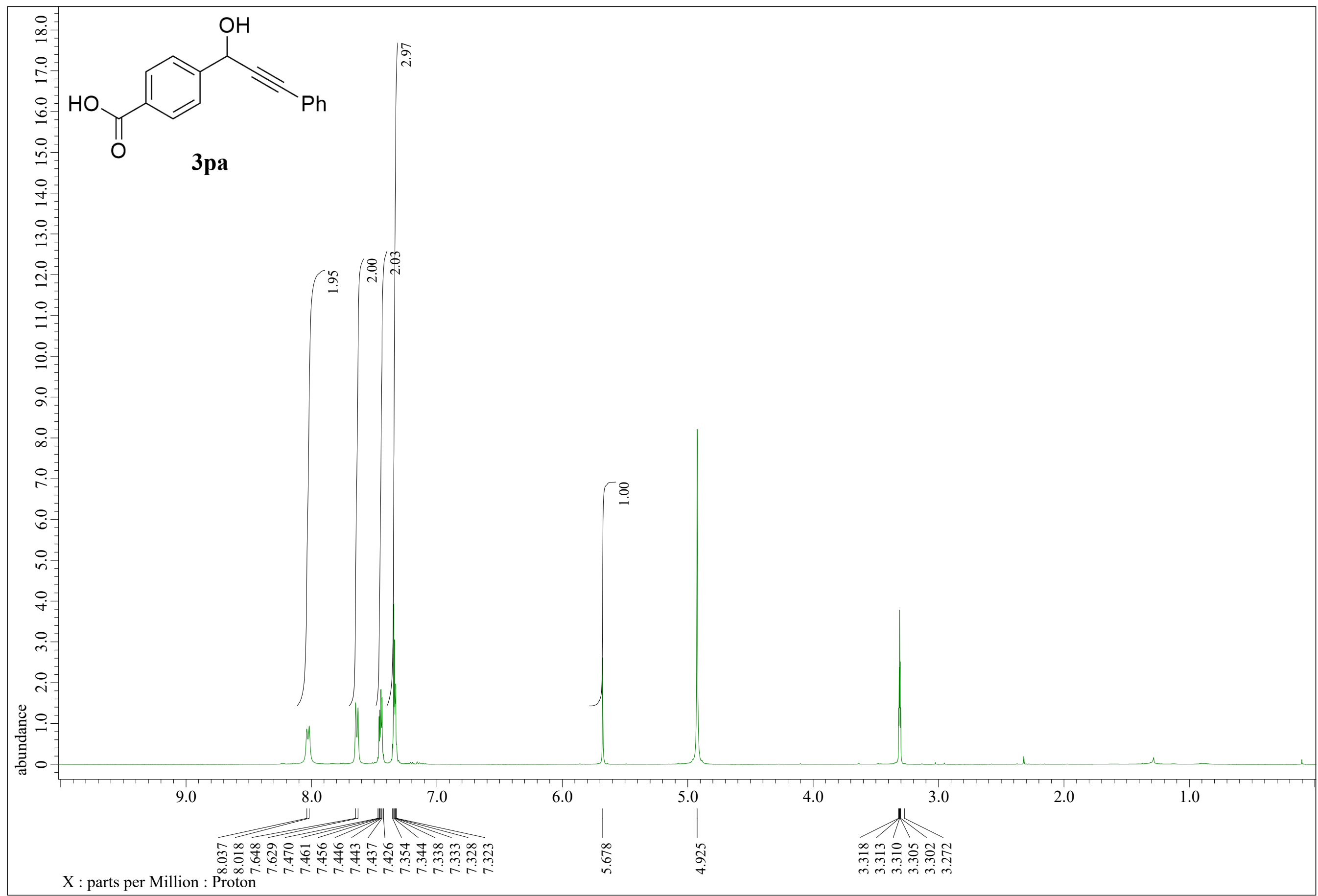


$100.5 \mathrm{MHz}, \mathrm{CD}_{3} \mathrm{OD}$

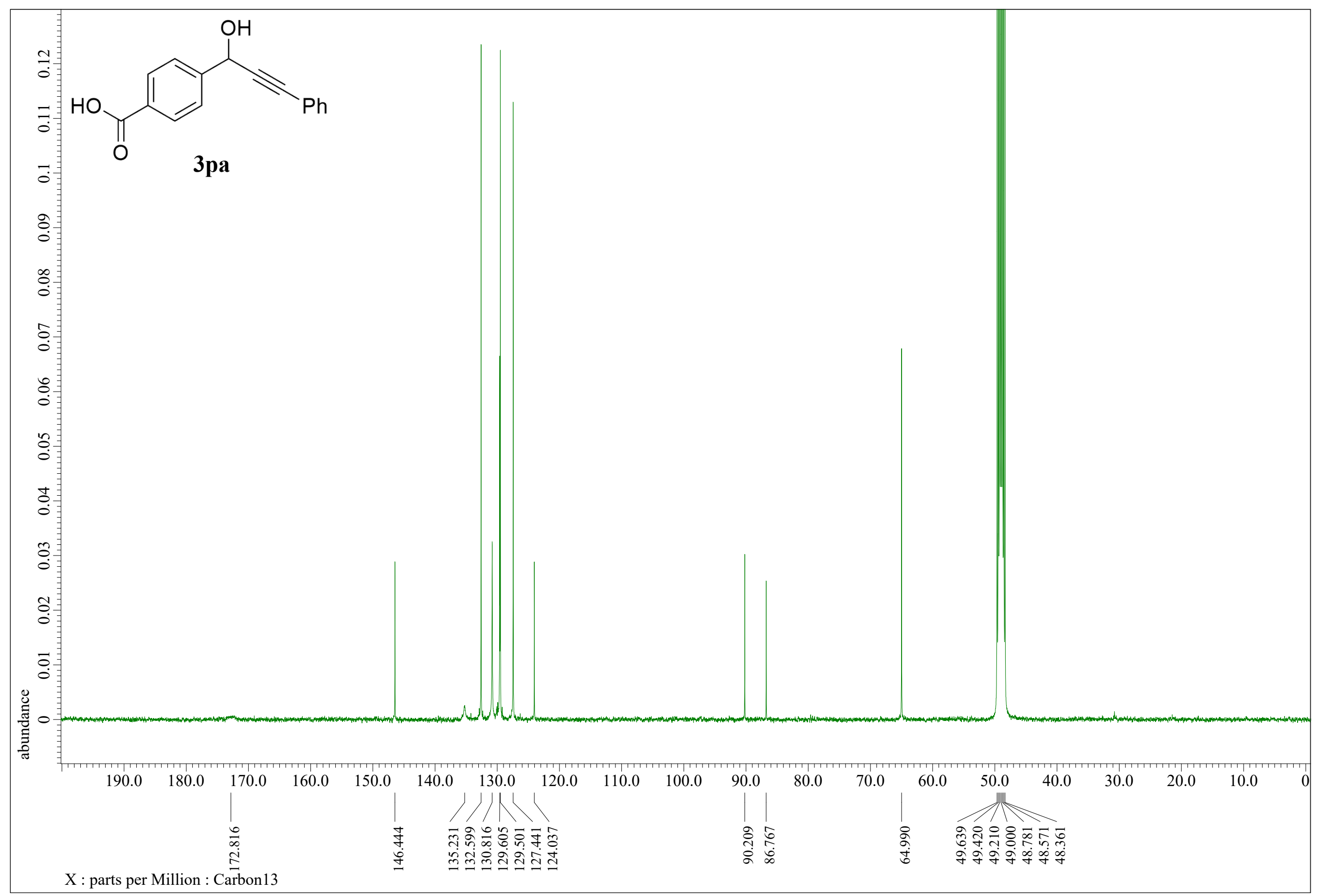


$400 \mathrm{MHz}, \mathrm{CDCl}_{3}$

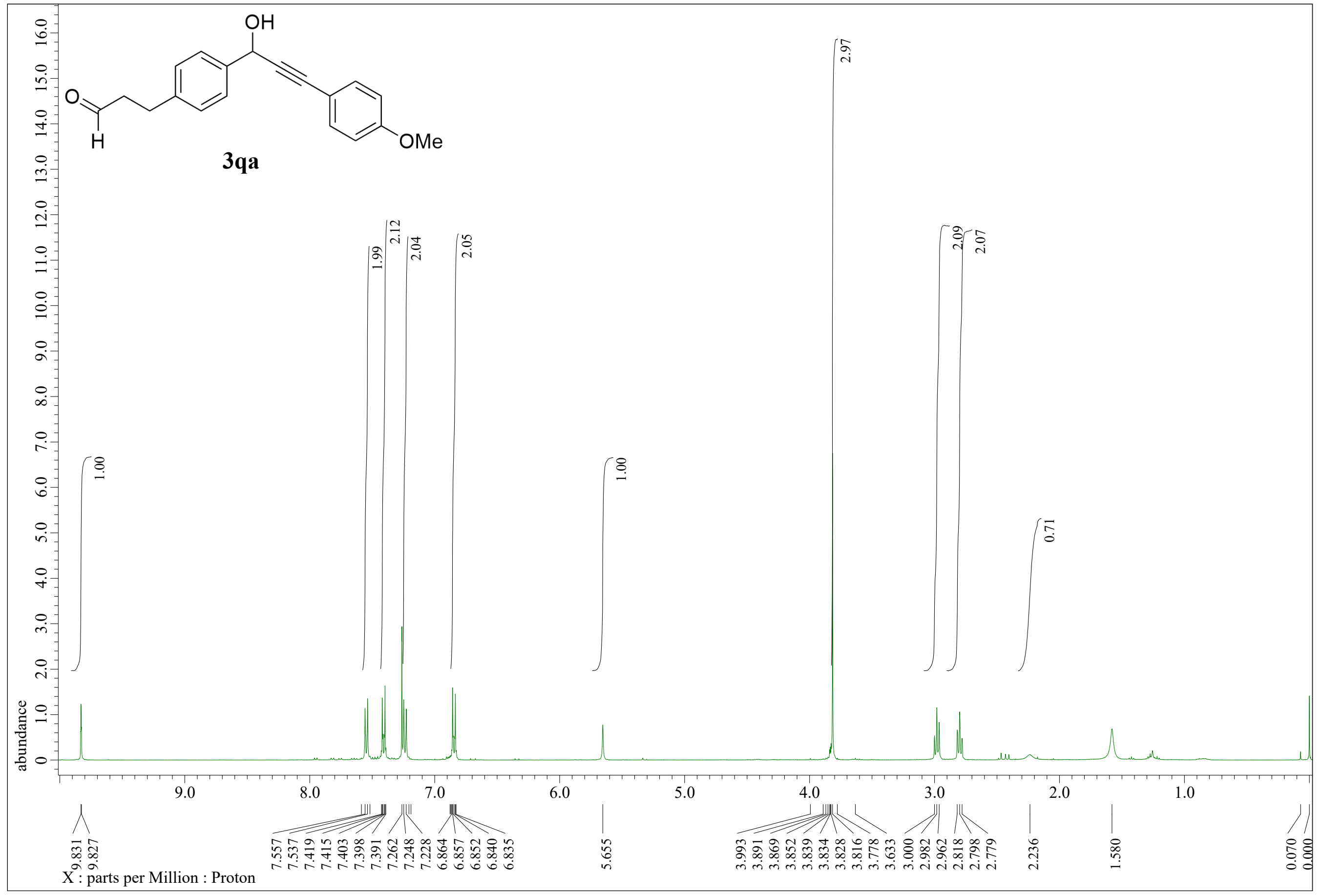


$100.5 \mathrm{MHz}, \mathrm{CDCl}_{3}$

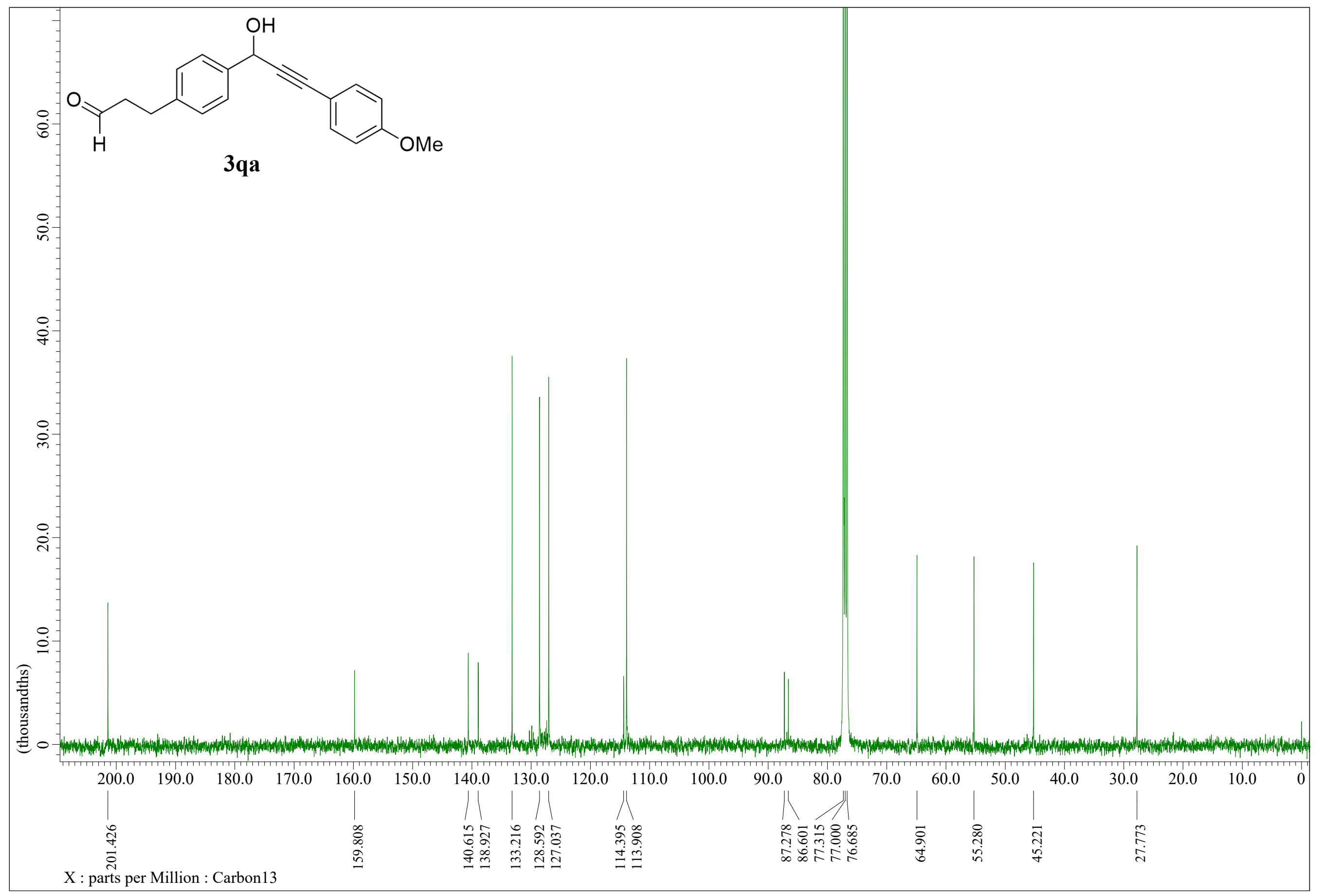


$400 \mathrm{MHz}, \mathrm{CDCl}_{3}$

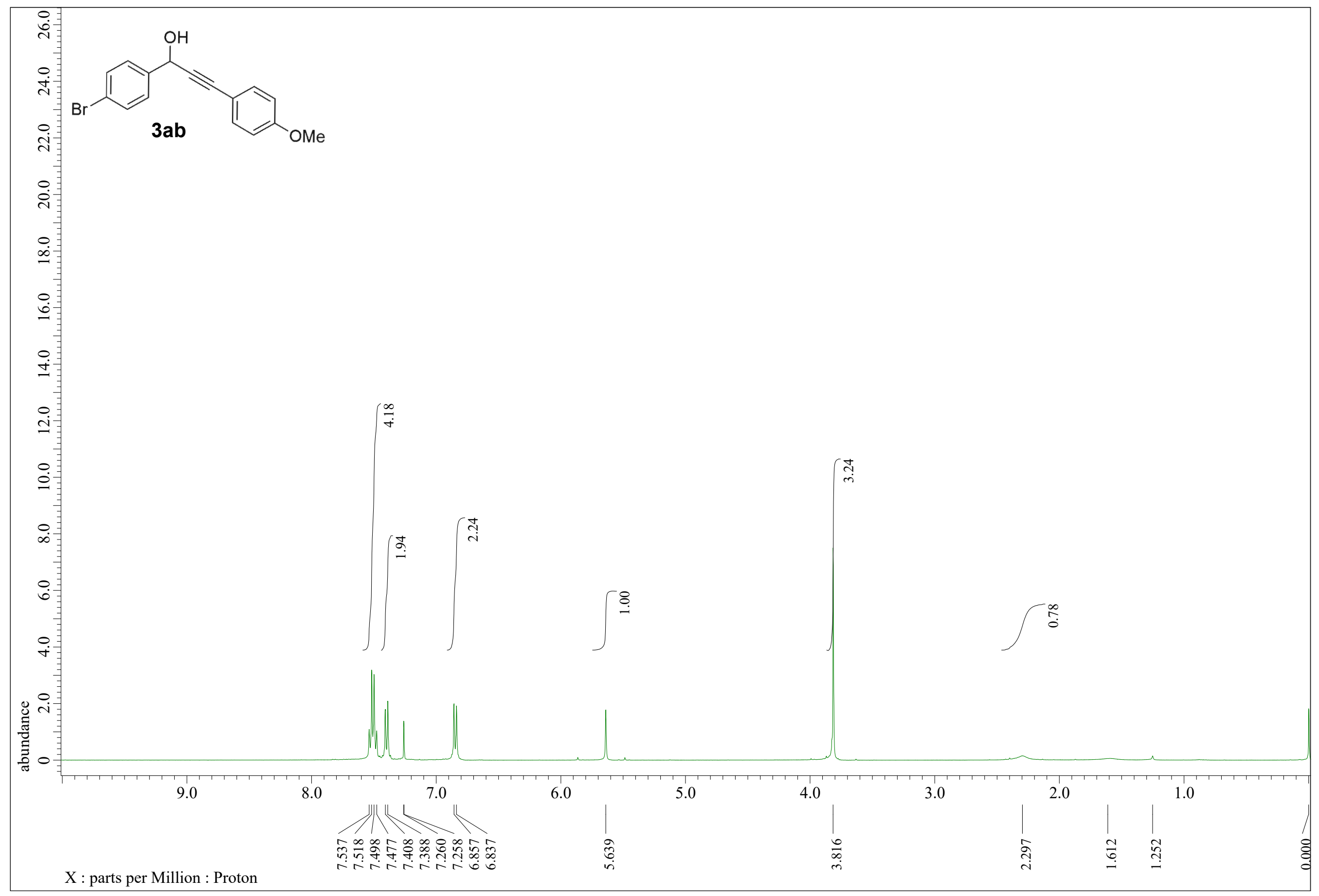


$100.5 \mathrm{MHz}, \mathrm{CDCl}_{3}$

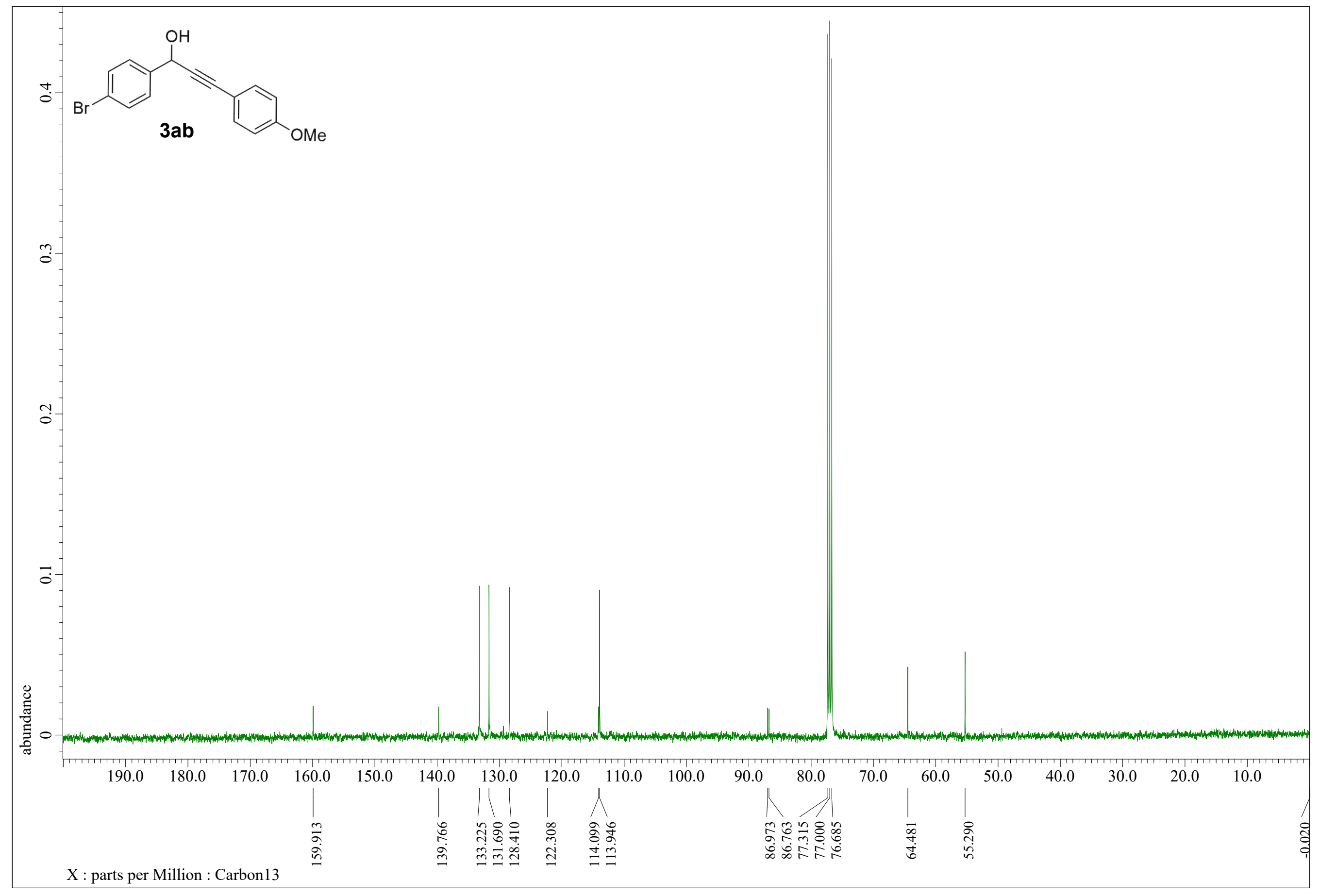


$400 \mathrm{MHz}, \mathrm{CDCl}_{3}$

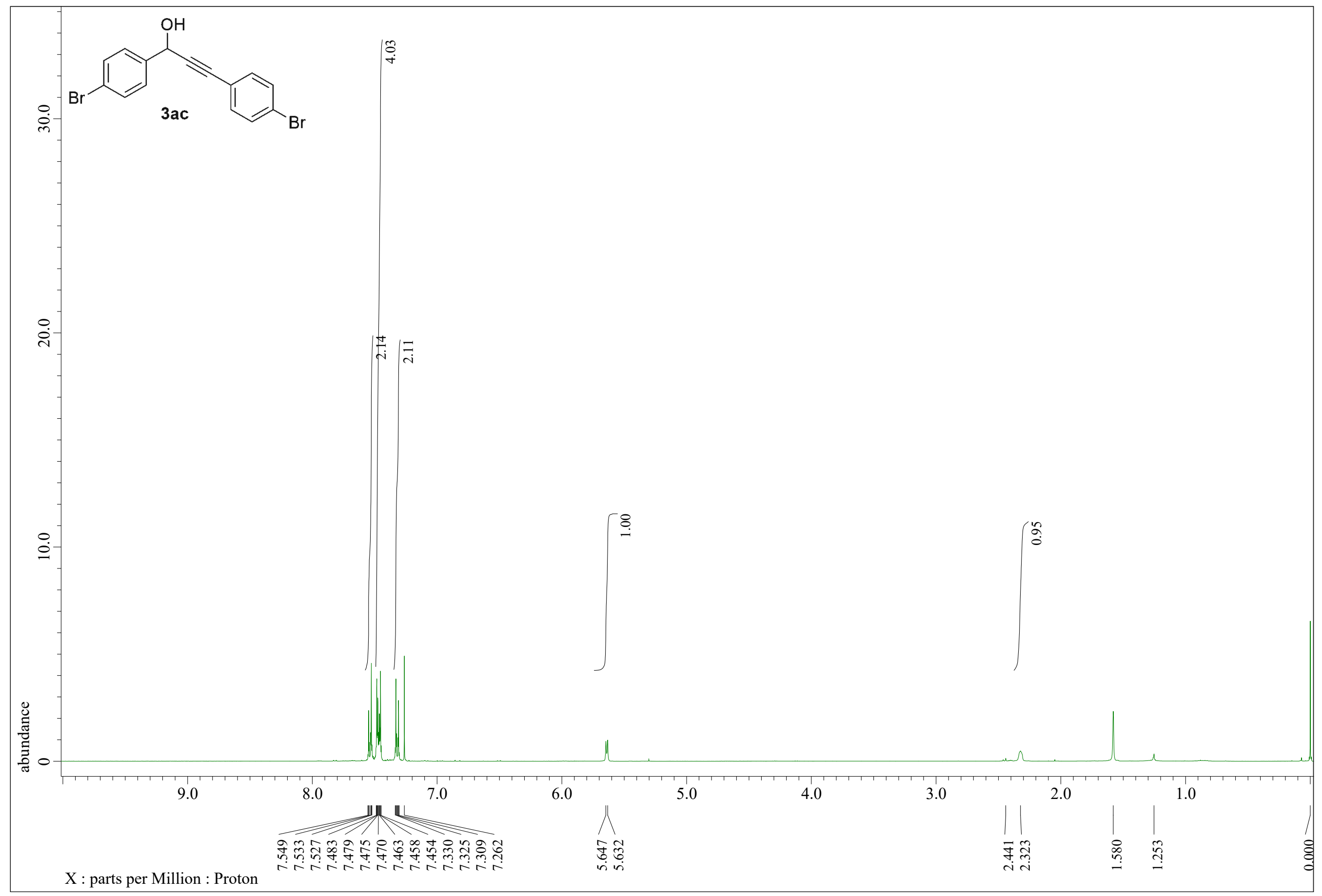


$100.5 \mathrm{MHz}, \mathrm{CDCl}_{3}$

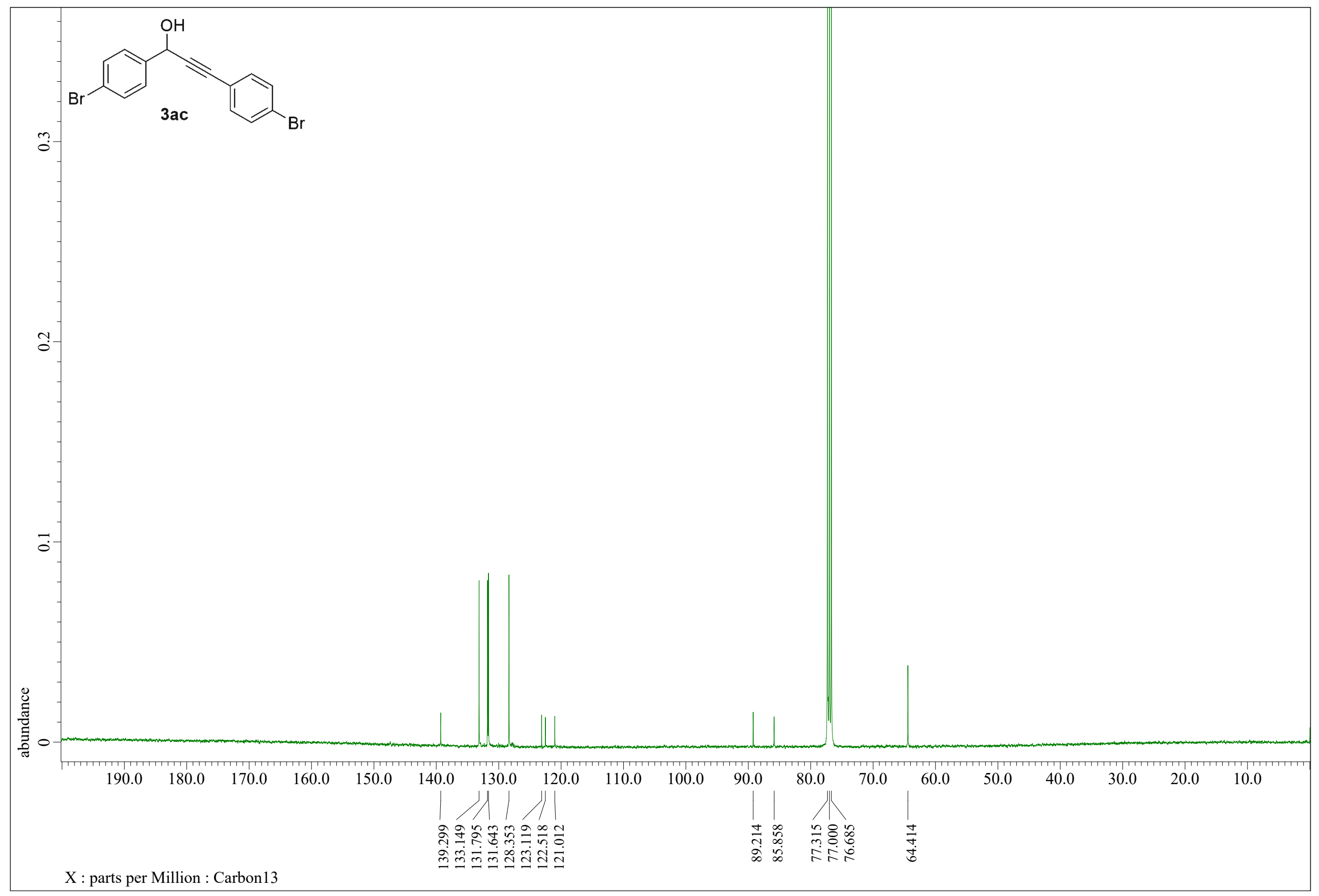


$400 \mathrm{MHz}, \mathrm{CDCl}_{3}$

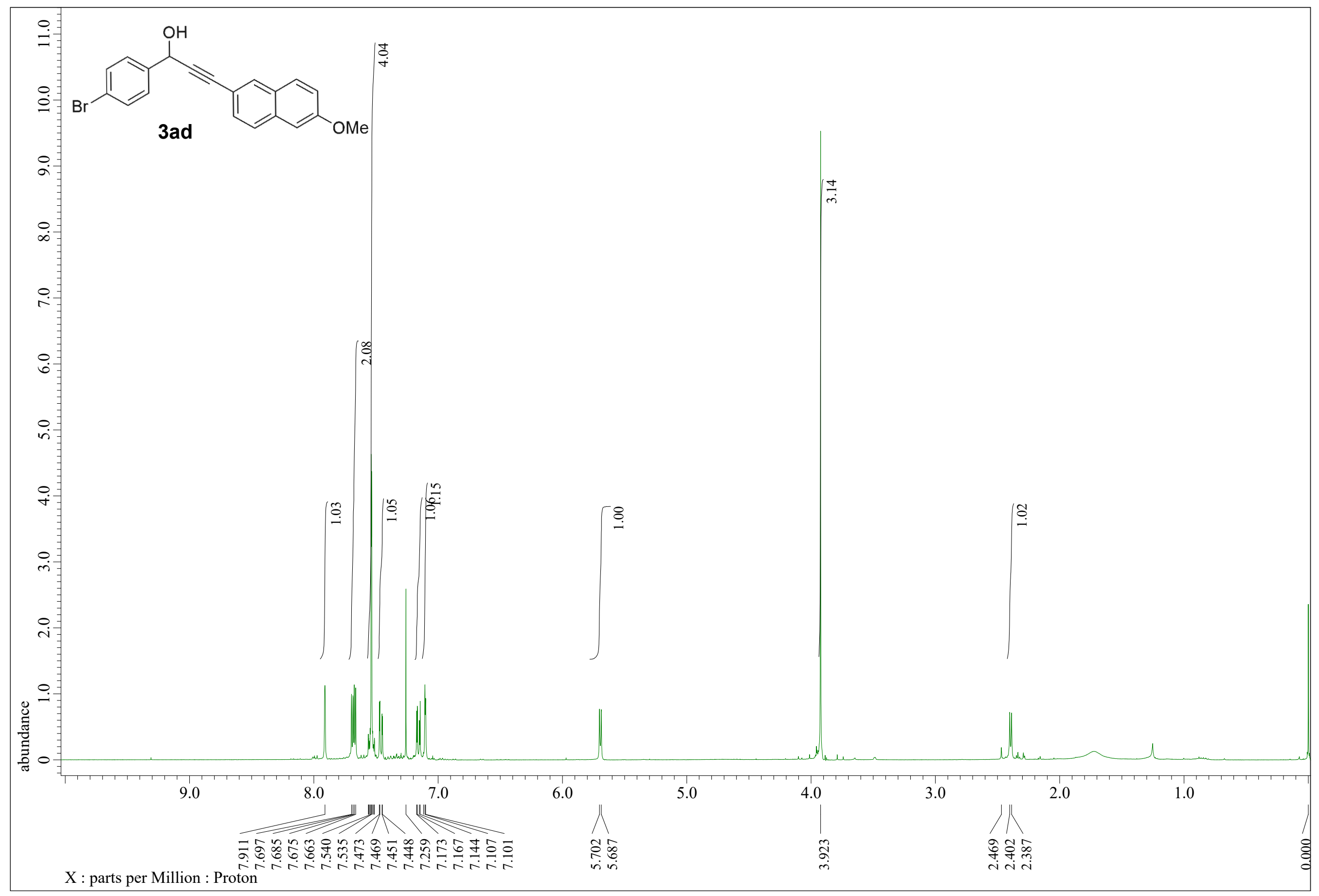


$400 \mathrm{MHz}, \mathrm{CDCl}_{3}$

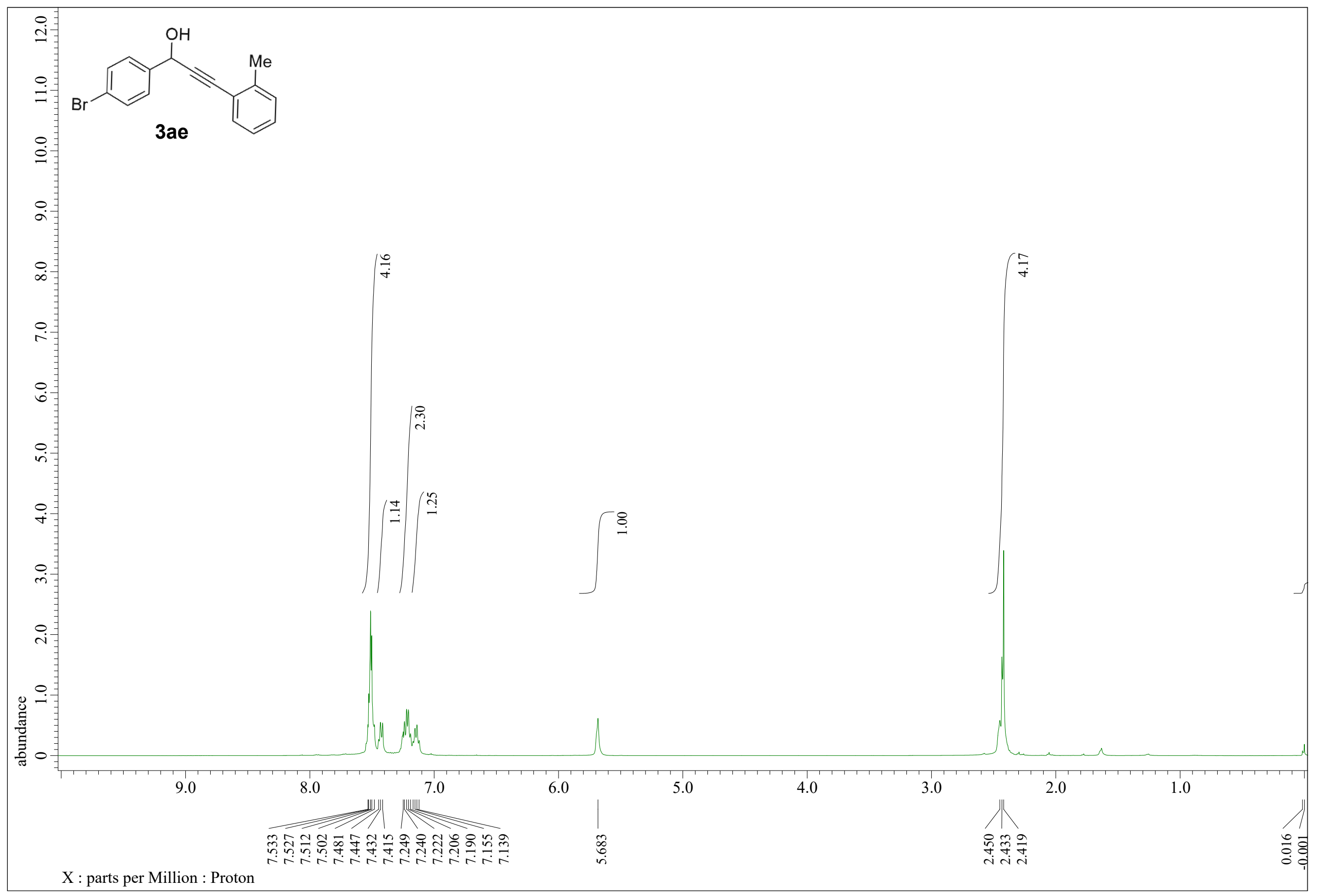


$100.5 \mathrm{MHz}, \mathrm{CDCl}_{3}$

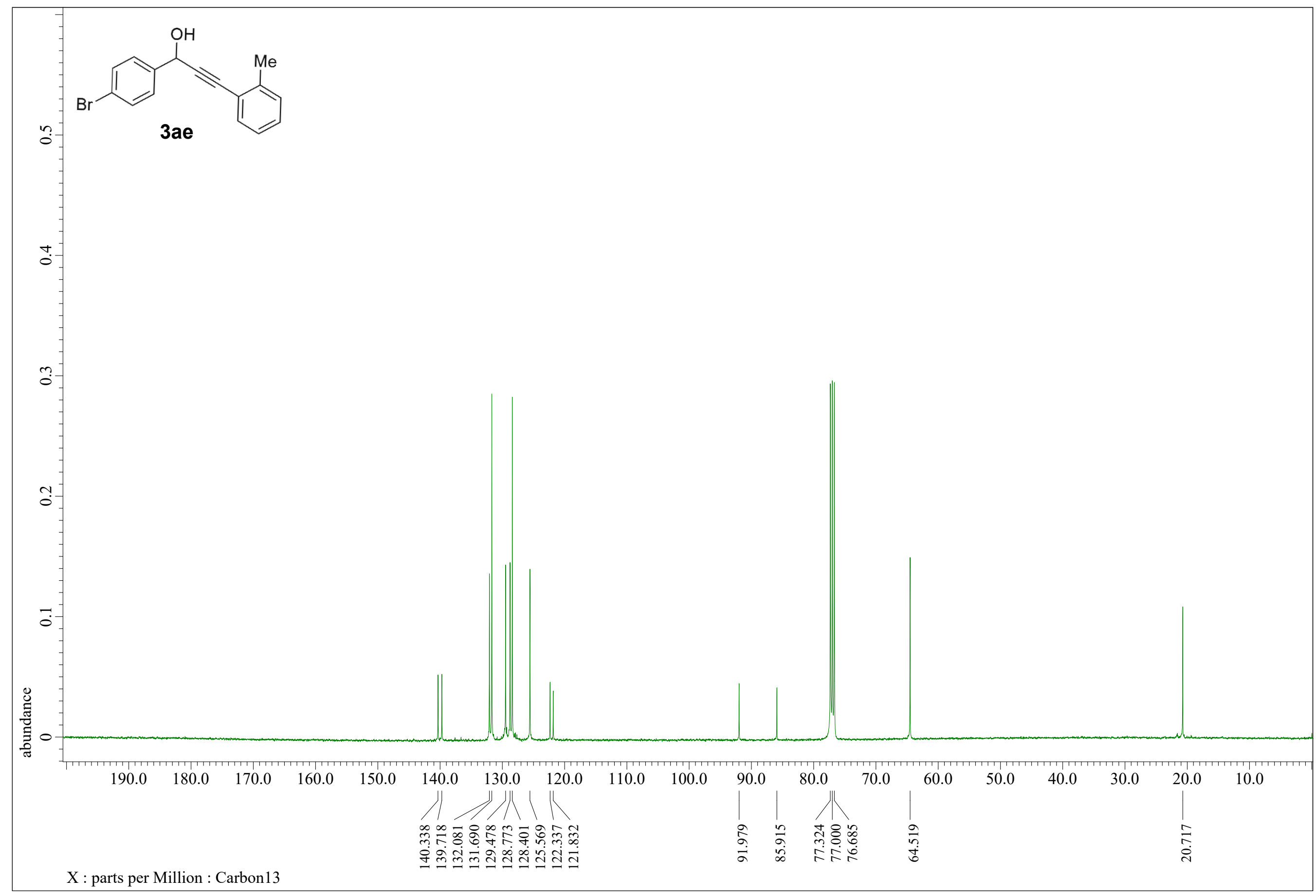


$400 \mathrm{MHz}, \mathrm{CDCl}_{3}$

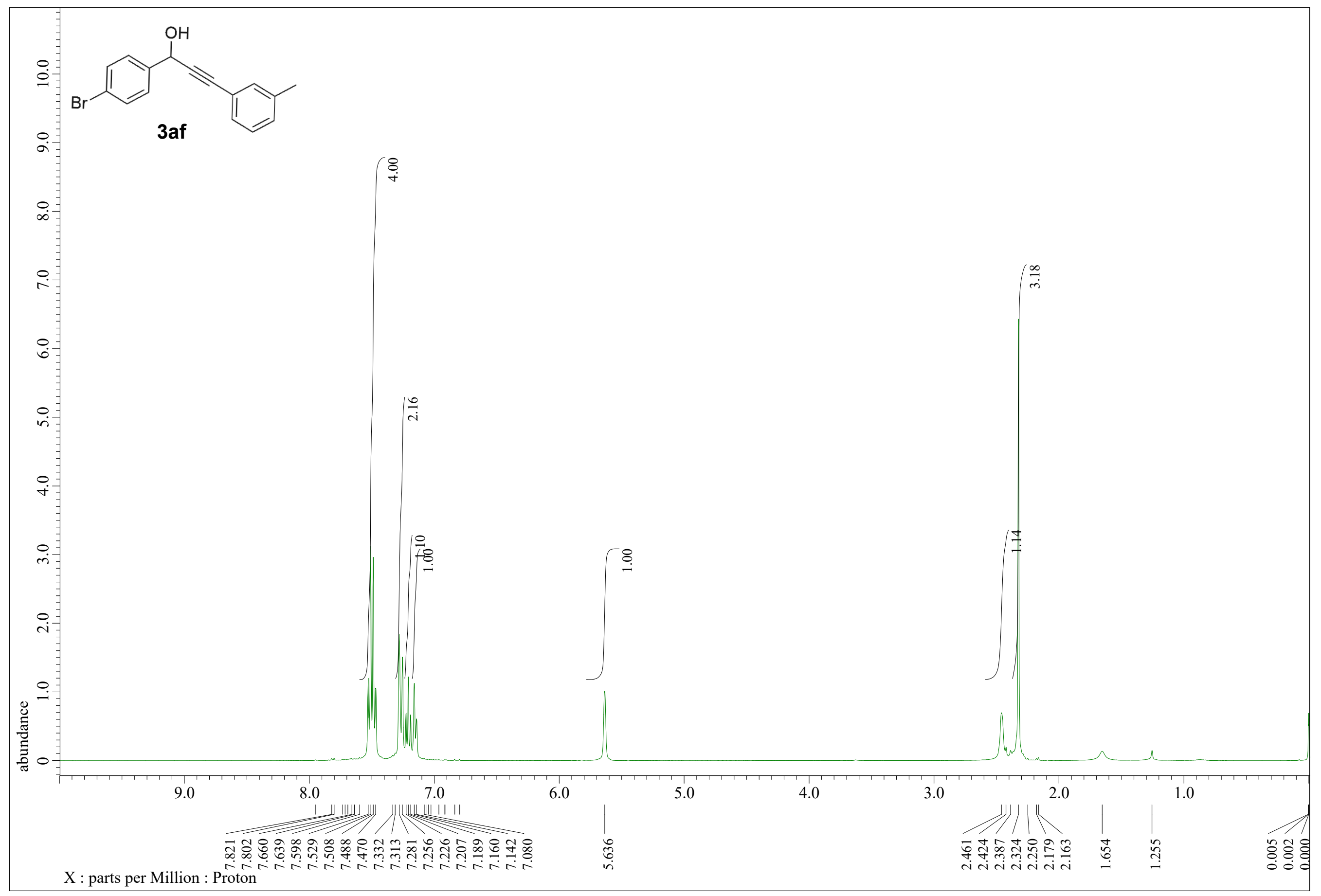


$100.5 \mathrm{MHz}, \mathrm{CDCl}_{3}$

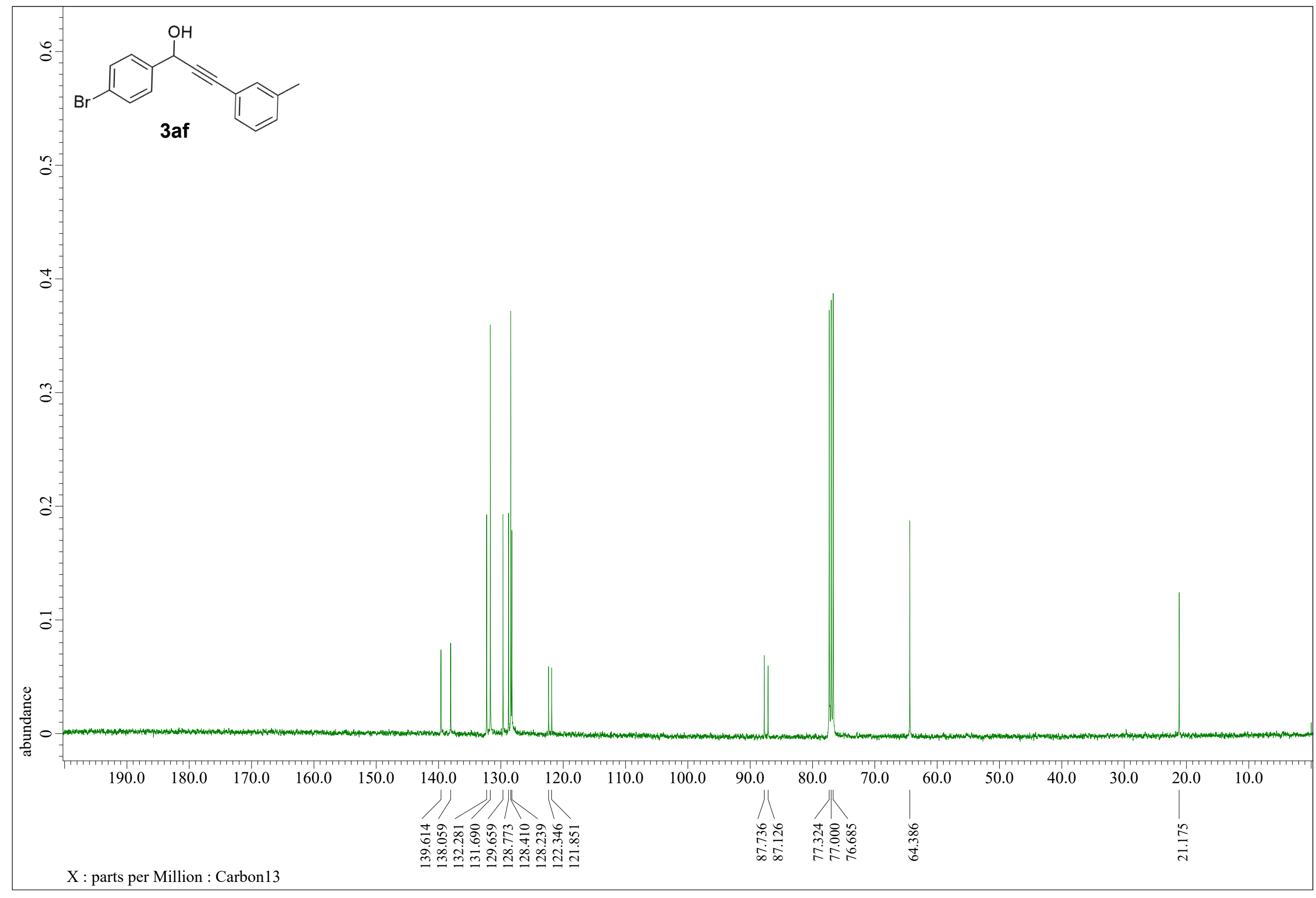




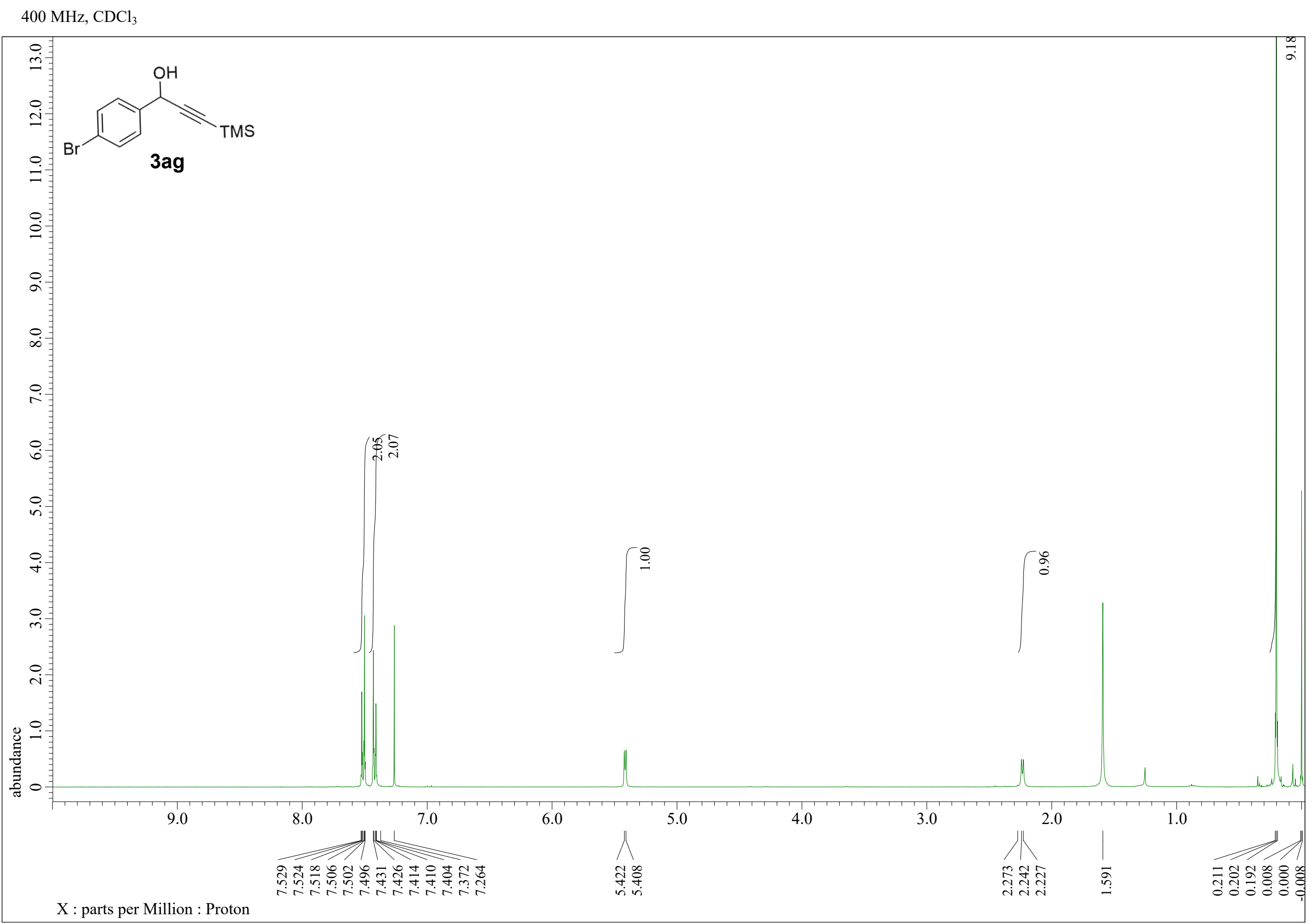


$100.5 \mathrm{MHz}, \mathrm{CDCl}_{3}$

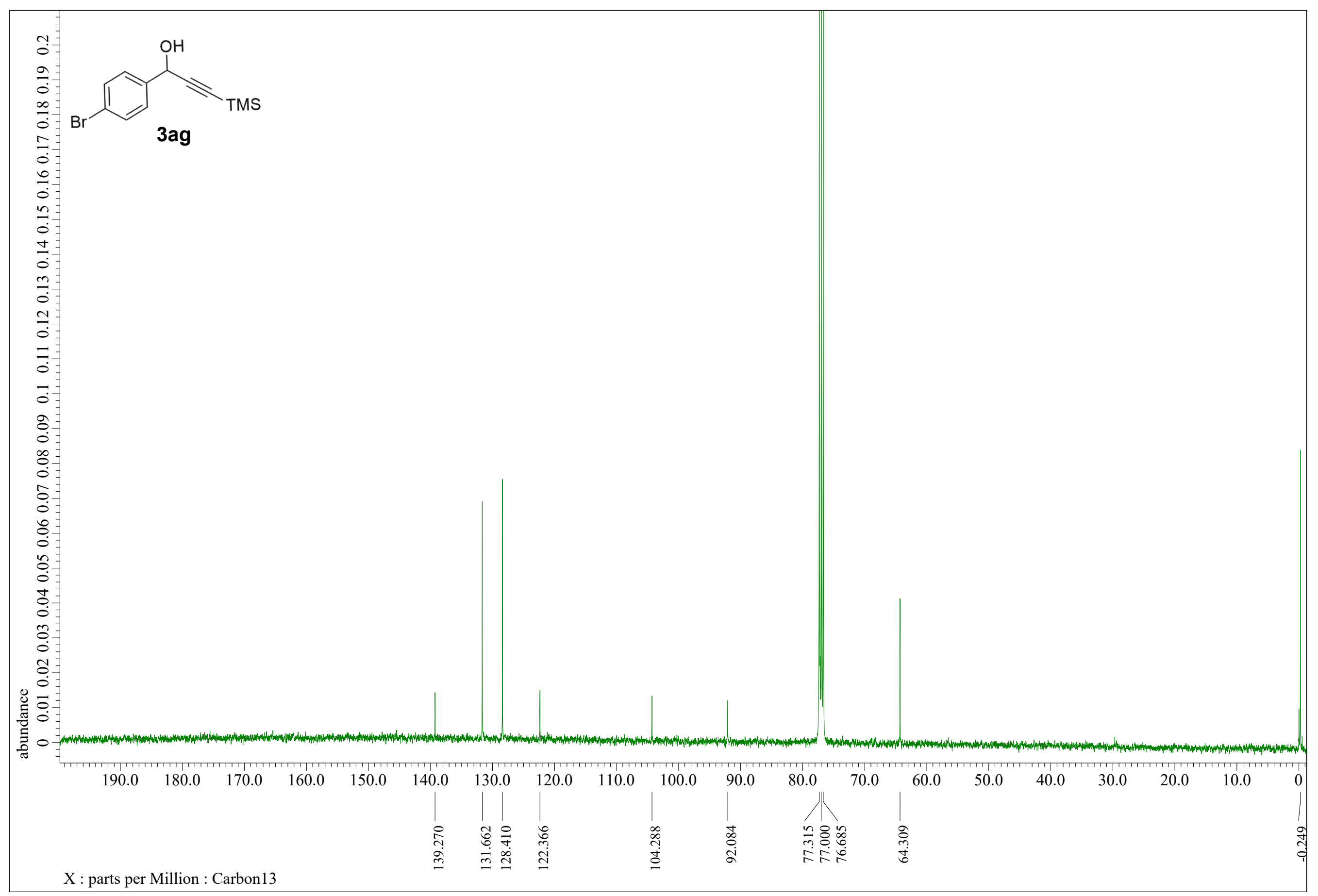

